\title{
INTERPLANETARY PROPAGATION OF SOLAR ENERGETIC PARTICLE HEAVY IONS OBSERVED AT 1 AU AND THE ROLE OF ENERGY SCALING
}

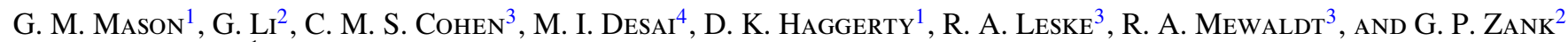 \\ ${ }^{1}$ Applied Physics Laboratory, Johns Hopkins University, Laurel, MD 20723, USA; glenn.mason@ @huapl.edu \\ ${ }^{2}$ Department of Physics, University of Alabama in Huntsville, Huntsville, AL 35805, USA \\ ${ }^{3}$ Department of Physics, California Institute of Technology, Pasadena, CA 91125, USA \\ ${ }^{4}$ Department of Space Science, Southwest Research Institute, San Antonio, TX 78228, USA \\ Received 2012 July 25; accepted 2012 October 27; published 2012 November 30
}

\begin{abstract}
We have studied $\sim 0.3$ to $>100 \mathrm{MeV}$ nucleon ${ }^{-1} \mathrm{H}, \mathrm{He}, \mathrm{O}$, and $\mathrm{Fe}$ in 17 large western hemisphere solar energetic particle events (SEP) to examine whether the often observed decrease of $\mathrm{Fe} / \mathrm{O}$ during the rise phase is due to mixing of separate SEP particle populations, or is an interplanetary transport effect. Our earlier study showed that the decrease in $\mathrm{Fe} / \mathrm{O}$ nearly disappeared if $\mathrm{Fe}$ and $\mathrm{O}$ were compared at energies where the two species interplanetary diffusion coefficient were equal, and therefore their kinetic energy nucleon ${ }^{-1}$ was different by typically a factor $\sim 2$ ("energy scaling"). Using an interplanetary transport model that includes effects of focusing, convection, adiabatic deceleration, and pitch angle scattering we have fit the particle spectral forms and intensity profiles over a broad range of conditions where the $1 \mathrm{AU}$ intensities were reasonably well connected to the source and not obviously dominated by local shock effects. The transport parameters we derive are similar to earlier studies. Our model follows individual particles with a Monte Carlo calculation, making it possible to determine many properties and effects of the transport. We find that the energy scaling feature is preserved, and that the model is reasonably successful at fitting the magnitude and duration of the $\mathrm{Fe} / \mathrm{O}$ ratio decrease. This along with successfully fitting the observed decrease of the $\mathrm{O} / \mathrm{He}$ ratio leads us to conclude that this feature is best understood as a transport effect. Although the effects of transport, in particular adiabatic deceleration, are very significant below a few $\mathrm{MeV}$ nucleon $^{-1}$, the spectral break observed in these events at $1 \mathrm{AU}$ is only somewhat modified by transport, and so the commonly observed spectral breaks must be present at injection. For scattering mean free paths of the order of $0.1 \mathrm{AU}$ adiabatic deceleration is so large below $\sim 200 \mathrm{keV}^{\text {nucleon }}{ }^{-1}$ that ions starting with such energies at injection are cooled sufficiently as to be unobservable at $1 \mathrm{AU}$. Because of the complicating factors of different spectral break energies for different elements, it appears that SEP abundances determined below the break are least susceptible to systematic distortions.
\end{abstract}

Key words: interplanetary medium - Sun: coronal mass ejections (CMEs) - Sun: flares - Sun: particle emission

\section{INTRODUCTION}

Large solar energetic particle (SEP) events are the most powerful particle acceleration phenomenon in the solar system, and are capable of filling the inner heliosphere with radiation levels that can affect humans and space systems (see, e.g., reviews by Ramaty et al. 1980; Lin 1987; Reames 1999). Besides accelerating protons and electrons, SEPs accelerate heavy ions in significant quantities up to $\mathrm{Fe}$, and the heavy ion composition, energy distribution, time of arrival, and state of ionization carry information about the acceleration site seed material, and the physical processes of acceleration and transport away from the Sun (see reviews by McGuire 1983; Mason 1987; Klecker et al. 1998; Reames 1999). It is known from X- and $\gamma$-ray emission from magnetic loop footpoints that SEPs are sometimes accelerated and confined to erupting magnetic loops on the Sun (e.g., Hudson et al. 2004 and references therein), yet in other cases large interplanetary shocks associated with coronal mass ejections (CMEs) appear to be the accelerating agent (Mason et al. 1984; Cane 1988; Cane et al. 1988; Gosling 1993; Cliver et al. 2004). Because the acceleration takes place in the corona or near the Sun, it is generally not possible to identify the acceleration site unambiguously, and given the complexities of the events it is reasonable to assume that in some cases the energetic particles reaching $1 \mathrm{AU}$ contain mixtures of particles accelerated near the solar surface as well as by associated interplanetary shocks (Kallenrode et al. 1992; Kocharov \& Torsti 2002; Cane et al. 2003; Li \& Zank 2005).

Heavy ion data in SEP events show complex behavior that leads to ambiguities in interpreting the data. For example, eventaveraged energy spectra show different shapes for different elements and so the relative abundances depend on particle energy for a given SEP event, and also show significant variations from one event to another (McGuire et al. 1986; Mazur et al. 1992; Cohen et al. 2005; Desai et al. 2006). Even during individual SEP events, ion abundances show systematic variations in time, for example, the $\mathrm{Fe} / \mathrm{O}$ ratio is often larger during the event rise phase than later (e.g., Scholer et al. 1978; von Rosenvinge \& Reames 1979; Mason et al. 1983; Tylka et al. 1999; Dietrich \& Tylka 2001). Numerous processes have been suggested to explain these features including details of the acceleration mechanism, geometry of the accelerating shock, mixtures of seed populations, and interplanetary transport (e.g., work cited above and Reames et al. 2000; Li et al. 2005; Tylka et al. 2005; Li et al. 2009b).

Most analysis of SEP energy spectra organizes the data by $\mathrm{MeV}$ nucleon $^{-1}$, comparing particles of the same speed. This is due in part to the analogy with galactic cosmic rays whose spectra are organized by velocity (e.g., George et al. 2009, and references therein), and also to practical considerations wherein most instruments measure different heavy ions over similar velocity ranges. Other organizing parameters such as magnetic 


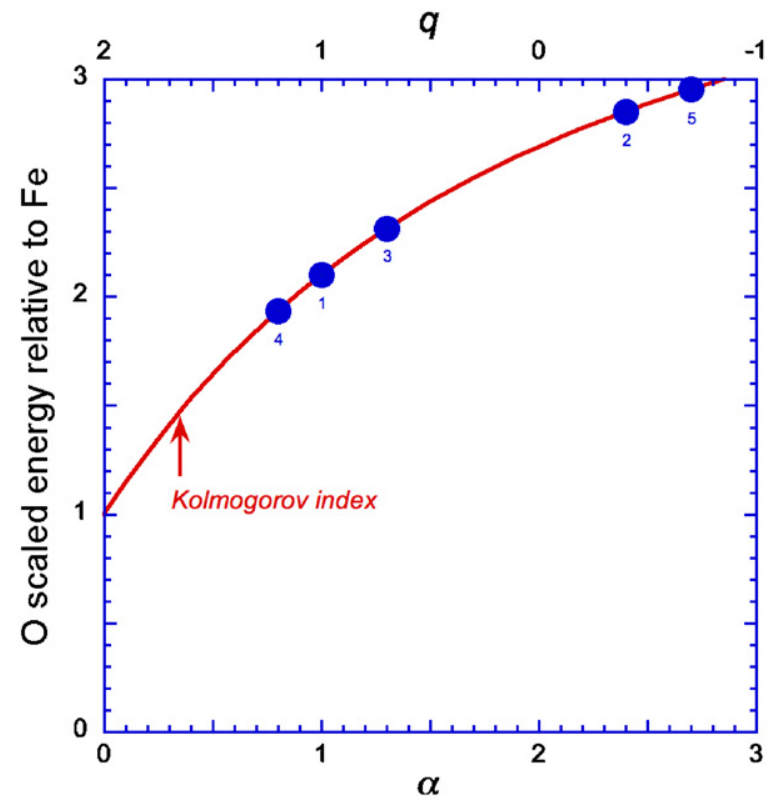

Figure 1. Energy scaling factor for O compared to Fe for particle mean free path varying as $\lambda \sim P^{\alpha}$, where $P$ is magnetic rigidity. Points 1-5 are scaling factors for five solar energetic particle events from Cohen et al. (2005). Red arrow marked Kolmogorov index shows scaling factor for interplanetary turbulence following a $q=5 / 3$ power law (see the text).

rigidity (momentum per unit charge) have been examined but without achieving a compellingly significant improvement in organizing the data (McGuire et al. 1986; Tylka et al. 1999; Desai et al. 2006). Recent studies using instruments on the Advanced Composition Explorer (ACE) satellite have extended the energy range of SEP heavy ion spectra well beyond previous work, yielding new insights into spectral properties. For example, Cohen et al. (2005; see also Mewaldt et al. 2005b; Cohen et al. 2007) examined particle spectra in several large SEP events, and found that spectra which showed different shapes when organized by velocity, became much more uniform in shape when plotted versus a "scaled energy" defined as the energy where the two species diffusion coefficients were equal. For example, for species $i$ with energy $E_{i} \mathrm{MeV}$ nucleon $^{-1}$, the scaled energy factor for $O$ is

$$
E_{O} E_{i}=\left[\frac{(Q / M)_{O}}{(Q / M)_{i}}\right]^{2 \alpha /(\alpha+1)}
$$

where $(Q / M)$ is the particle charge-to-mass ratio. In the exponent, $\alpha$ is the rigidity dependence of the scattering mean free path, which in turn is related to the power spectrum of magnetic turbulence $I(k) \sim k^{-q}$ as $\alpha=2-q$ (Dröge 1994). For typical O and Fe ionization states in SEP events, and $\alpha=1$, Equation (1) gives a scaled energy of $\sim 2.0$ for $\mathrm{O}$ compared to Fe (Cohen et al. 2005) as shown in Figure 1. (Sometimes workers compare particles at equal rigidity which is equivalent to a scaling factor $\sim 2$ times larger than deduced from Equation (1) for $\alpha=1$ ).

A study of the temporal behavior of $\mathrm{O}$ and $\mathrm{Fe}$ in large SEP events by Mason et al. (2006) showed that the hourly averaged intensities of $\mathrm{O}$ and $\mathrm{Fe}$ were much more similar when compared at scaled energies than at the same energy per nucleon, especially during the period when intensities rise to maximum (rise phases). In that work 14 large western hemisphere events were studied. It was found that if one compared the Fe intensity at $276 \mathrm{keV}^{\text {nucleon }}{ }^{-1}$ and $13.2 \mathrm{MeV}^{\text {nucleon }}{ }^{-1}$ with $\mathrm{O}$ at a scaled energy, most of the temporal variation of the $\mathrm{Fe} / \mathrm{O}$ ratio (at the same energy nucleon ${ }^{-1}$ ) was removed. That is, the scaled energy intensity versus time profiles were nearly the same. The energy scaling parameter was separately estimated for each of the 14 events, and showed a range from 1.0 to 2.8 , with 2.0 being typical.

If energy scaling leads to a superior ordering of SEP temporal and spectral abundances, then it could be that SEP properties should be evaluated with scaled energies rather than the same energy per nucleon. This could have important impact on key properties discussed above, namely if SEP heavy ions are well ordered by scaled energies instead of energy per nucleon, then

1. an enhanced $\mathrm{Fe} / \mathrm{O}$ ratio early in SEP events would be due to transport effects, and not to mixture of seed populations, or a preceding SEP event that happens to be enhanced in Fe;

2. the relative abundances of SEPs should be calculated by comparing at the same scaled energies, possibly leading to a substantial revision of abundances computed at the same energy per nucleon;

3. energy spectra should be displayed versus scaled energy, lessening or perhaps removing the differences in spectral slopes compared at the same energy nucleon ${ }^{-1}$ (Mewaldt et al. 2005a; Li et al. 2009b);

4. scattering in the interplanetary medium would be identified as an important process in many large SEP events, and distinguished from other possible competing mechanisms such as delayed acceleration or release from sites near the Sun (Mason et al. 2006).

However, these possible conclusions are all based on the simple arguments involving diffusive processes. In the case of SEP transport, it has long been known that other effects such as convection, adiabatic deceleration, and magnetic focusing are key parts of the physics, so it is unclear if energy scaling would emerge from a more complete treatment of the particle transport. The purpose of this paper is to report such an investigation using a detailed transport model based on properties of interplanetary turbulence, and adding $\mathrm{H}$ and $\mathrm{He}$ intensities to the $\mathrm{O}$ and $\mathrm{Fe}$ used previously to cover a broader range of energies and chargeto-mass ratios. We find that the energy scaling property also emerges from the complete transport model, and that many features of SEP intensities at $1 \mathrm{AU}$ are controlled by transport while at the same time in many cases other mechanisms such as confinement by accelerating shocks and connection effects are also important, especially at low energies. Implications for solar source spectra and the impact on deduced relative abundances are also examined.

\section{OBSERVATIONS}

\subsection{Instrumentation}

Heavy ion intensities of $\mathrm{He}, \mathrm{O}$, and $\mathrm{Fe}$ were measured using the high sensitivity instruments on the $A C E$ spacecraft (Stone et al. 1998b). Below a few MeV nucleon ${ }^{-1} \mathrm{He}-\mathrm{Fe}$ data were from the Ultra Low Energy Isotope Spectrometer (ULEIS; Mason et al. 1998), and higher energies were from the Solar Isotope Spectrometer (SIS; Stone et al. 1998a). In order to add $\mathrm{H}$ to our study it was necessary to use data from additional instruments. Proton intensities below a few $\mathrm{MeV}$ were from the $A C E$ Electron, Proton, and Alpha Monitor (Gold et al. 1998), and at higher energies were from NOAA's Geosynchronous Operational Environmental Satellites (GOES; Onsager et al. 1996). For each event, hourly average intensities 
Table 1

Flare Properties

\begin{tabular}{|c|c|c|c|c|c|c|c|}
\hline \multirow[b]{2}{*}{$\begin{array}{l}\text { Event No. } \\
\text { (1) }\end{array}$} & \multirow[b]{2}{*}{$\begin{array}{l}\text { Year } \\
(2)\end{array}$} & \multicolumn{5}{|c|}{ Flare $^{\mathrm{a}}$} & \multirow[b]{2}{*}{$\begin{array}{c}\text { Peak Proton Flux } \\
\text { (8) }\end{array}$} \\
\hline & & $\begin{array}{l}\text { Date } \\
\text { (3) }\end{array}$ & $\begin{array}{l}\text { Day of Year } \\
\text { (4) }\end{array}$ & $\underset{(5)}{\operatorname{Maximum}}(\mathrm{UT})$ & $\begin{array}{c}\text { X-Ray Class/Importance } \\
\text { (6) }\end{array}$ & $\begin{array}{l}\text { Location } \\
\text { (7) }\end{array}$ & \\
\hline 1 & 1998 & May 6 & 126 & 0809 & $\mathrm{X} 2 / 1 \mathrm{~N}$ & S11W65 & 210 \\
\hline 2 & 1998 & Sep 30 & 273 & 1350 & $\mathrm{M} 2 / 2 \mathrm{~N}$ & N23W81 & 1200 \\
\hline 3 & 1999 & Jun 04 & 155 & 0703 & $\mathrm{M} 3 / 2 \mathrm{~B}$ & N17W69 & 64 \\
\hline 4 & 2000 & Sep 12 & 256 & 1213 & $\mathrm{M} 1 / 2 \mathrm{~N}$ & S17W09 & 320 \\
\hline 5 & 2000 & Oct 25 & 299 & 1125 & $\mathrm{C} 4$ & $\mathrm{~W} 50^{\mathrm{c}}$ & 15 \\
\hline 6 & 2001 & $\operatorname{Jan} 28$ & 28 & 1600 & $\mathrm{M} 1 / 1 \mathrm{~N}$ & S04W59 & 49 \\
\hline 7 & 2001 & Apr 15 & 105 & 1350 & $\mathrm{X} 14 / 2 \mathrm{~B}$ & S20W85 & 951 \\
\hline 8 & 2002 & Apr 21 & 111 & 0151 & $\mathrm{X} 1 / 1 \mathrm{~F}$ & S14W84 & 2520 \\
\hline 9 & 2002 & Jul 7 & 188 & 1143 & M1 & W limb & 22 \\
\hline 10 & 2002 & Aug 14 & 226 & 0212 & $\mathrm{M} 2 / 1 \mathrm{~N}$ & N09W54 & 26 \\
\hline 11 & 2002 & Aug 24 & 236 & 0112 & $\mathrm{X} 3 / 1 \mathrm{~F}$ & $\mathrm{~S} 02 \mathrm{~W} 81^{\mathrm{c}}$ & 317 \\
\hline 12 & 2003 & Nov 4 & 308 & 1929 & $\mathrm{X} 28 / 3 \mathrm{~B}$ & S19W83 & 353 \\
\hline 13 & 2003 & $\operatorname{Dec} 2$ & 336 & 0948 & C7 & S13W65 & 86 \\
\hline 14 & 2004 & Apr 11 & 102 & 0419 & $\mathrm{C} 9 / 1 \mathrm{~F}$ & S14W47 & 35 \\
\hline $15^{\mathrm{e}}$ & 2005 & Jan 20 & 20 & 0701 & $\mathrm{X} 7$ & N14W61 & 1860 \\
\hline 16 & 2006 & Dec 13 & 347 & 0240 & $\mathrm{X} 3 / 4 \mathrm{~B}$ & S05W23 & 698 \\
\hline 17 & 2012 & Jan 23 & 23 & 0359 & $\mathrm{M} 8 / 2 \mathrm{~B}$ & N28W36 & 6310 \\
\hline
\end{tabular}

Notes.

${ }^{a}$ Flare data and proton flux from NOAA site http://www.swpc.noaa.gov/ftpdir/indices/SPE.txt, except as noted.

${ }^{\mathrm{b}}$ Units: particles $\mathrm{s}^{-1} \mathrm{~cm}^{2}$ sr above $10 \mathrm{MeV}$.

${ }^{\mathrm{c}}$ Flare location from Kahler (2005).

${ }^{\mathrm{d}}$ Flare location from Cane et al. (2006).

${ }^{\mathrm{e}}$ Last in a sequence of three events that occurred between 2005 January 16 and January 20; from Desai et al. (2006).

from $\sim 0.3 \mathrm{MeV}$ nucleon $^{-1}$ to $\sim 100 \mathrm{MeV}$ nucleon $^{-1}$ were obtained with the upper energy limit determined by counting statistics; this corresponds to a rigidity range of $\sim 25$ to $>2500 \mathrm{MV}$, much larger than most previous studies of ion propagation in interplanetary space.

\subsection{Event Selection}

We used the 14 large SEP events from our earlier study (Mason et al. 2006) and added three additional ones: 1998 May 6 (Day of Year 126, hereafter DOY) due to its relatively low scattering effects in interplanetary space and unusually large ${ }^{3} \mathrm{He} /{ }^{4} \mathrm{He}$ ratio (Cohen et al. 1999), and two well-studied events that occurred after the end of the prior study: 2006 December 13 (DOY 347) and 2012 January 23 (DOY 23). In order to minimize effects due to magnetic connection, all 17 events studied here had parent activity in the solar western hemisphere. All were associated with shocks or CMEs and are included on the NOAA Space Environment Center list of $>10 \mathrm{MeV}$ proton events. ${ }^{5}$ Table 1 lists flare properties of the selected events: Columns 2-4 list the flare maximum year and day, Column 5 the flare maximum time, Column 6 the X-ray class and optical importance, Column 7 the location, and Column 8 the peak proton intensity for energies $>10 \mathrm{MeV}$. Table 2 lists selected energetic particle properties for the events: Columns 2 and 3 are the flare date and DOY; Column 4 the decimal DOY of shock passage; Column 5 the approximate highest energy for $\mathrm{O}$ for which the shock passage is associated with an intensity increase; Columns 6-9 the approximate factor of decrease of $\mathrm{He} / \mathrm{H}$ and $\mathrm{Fe} / \mathrm{O}$ associated with the rise phase; Columns 10 and 11 the energy scaling ratio (discussed below) that removes most of the rise phase ratio change; Column 12 the

\footnotetext{
5 See http://www.swpc.noaa.gov/ftpdir/indices/SPE.txt.
}

O spectral break energy; Column 13 the event-averaged Fe/O at $0.32-0.45 \mathrm{MeV}^{\text {nucleon }}{ }^{-1}$; Column 14 notes whether or not ${ }^{3} \mathrm{He}$-rich material was clearly present during the day before the event onset. The selected events represent about one-quarter of the western hemisphere events on the SEC list since $A C E$ was launched, and were intended to be a large enough sample so that the deduced properties can be considered representative. Many of these events have been the subject of studies in the published literature. Some large well-studied events (e.g., 2000 July 14, 2001 November 4) were avoided since they caused instrument saturation.

Figure 2 illustrates different types of SEP events for the heavy ions discussed here. Figures 2(a)-(c) show particle arrival spectrograms of 6-80 AMU ions plotting 1/ion speed versus time, which for pure velocity dispersion propagation along a typical 1.2 AU field line from the Sun will produce particle arrival times along the red diagonal lines in the panels. The spectrogram color scales peak at red for the most intense, but scales are separate for each plot. Panels (d)-(f) show hourly averaged $\mathrm{O}$ and $\mathrm{Fe}$ intensities at $386 \mathrm{keV}^{\text {nucleon }}{ }^{-1}$. Panels (g)-(i) repeat the hourly averaged $\mathrm{Fe}$ intensities from the middle row and also show hourly averaged $\mathrm{O}$ intensities at higher energies than the middle panel $\mathrm{O}$.

The three columns in Figure 2 illustrate different SEP event behaviors routinely observed by the ULEIS instrument. Panels (a), (d), and (g) show a narrow pulse of heavy ions with arrival times consistent with essentially pure velocity dispersion from the Sun along a 1.2 AU nominal field line with release at the time of the associated X-ray flare (Kahler et al. 2001). Fe and $\mathrm{O}$ ions in this event arrived simultaneously, as can be seen from the middle panel where the two profiles overlap at the same energy nucleon ${ }^{-1}$ (Mason et al. 2004). For the 2000 event if we compare the Fe profile from the middle panel with higher 
Table 2

SEP Event Propertie

\begin{tabular}{|c|c|c|c|c|c|c|c|c|c|c|c|c|c|}
\hline \multirow[b]{2}{*}{ Event No. } & \multirow[b]{2}{*}{$\begin{array}{c}\text { Date of } \\
\text { Maximum }^{\mathrm{a}} \\
\text { (2) }\end{array}$} & \multirow[b]{2}{*}{$\begin{array}{l}\text { DOY }^{\mathrm{a}} \\
\text { (3) }\end{array}$} & \multirow[b]{2}{*}{$\begin{array}{l}\text { DOY Shock } \\
\text { Passage } \\
\text { (4) }\end{array}$} & \multirow[b]{2}{*}{$\begin{array}{c}\text { Shock Energy } \\
\text { Limit }^{c} \\
\text { (5) }\end{array}$} & \multicolumn{4}{|c|}{ Rise Phase-related Ratio Changes } & \multirow[b]{2}{*}{$\begin{array}{c}\text { Energy Scaling } \\
\text { Low }^{\mathrm{h}} \\
(10)\end{array}$} & \multirow[b]{2}{*}{$\begin{array}{c}\text { Energy Scaling } \\
\text { High }^{\mathrm{i}} \\
(11)\end{array}$} & \multirow[b]{2}{*}{$\begin{array}{l}\text { O Spectral } \\
\text { Break }^{\mathrm{j}} \\
(12)\end{array}$} & \multirow[b]{2}{*}{$\begin{array}{c}\text { Event-averaged } \\
\mathrm{Fe} / \mathrm{O}^{\mathrm{k}} \\
(13)\end{array}$} & \multirow[b]{2}{*}{$\begin{array}{c}\text { Preceded by }{ }^{3} \mathrm{He} \text {-rich } \\
\text { Material? } \\
(14)\end{array}$} \\
\hline & & & & & $\begin{array}{l}\mathrm{He} / \mathrm{H} \\
\text { Low }^{\mathrm{d}} \\
(6)\end{array}$ & $\begin{array}{c}\mathrm{He} / \mathrm{H} \\
\mathrm{High}^{\mathrm{e}} \\
(7)\end{array}$ & $\begin{array}{l}\mathrm{Fe} / \mathrm{O} \\
\mathrm{Low}^{\mathrm{f}} \\
(8)\end{array}$ & $\begin{array}{l}\mathrm{Fe} / \mathrm{O} \\
\mathrm{High}^{\mathrm{g}} \\
(9)\end{array}$ & & & & & \\
\hline 1 & 1998 May 6 & 126 & .. & $\ldots$ & 4 & 4 & 4 & 5 & 1.4 & 1.5 & 10.4 & $0.830 \pm 0.034$ & Yes \\
\hline 2 & 1998 Sep 30 & 273 & 275.29 & 0.14 & 8 & 10 & 20 & 10 & 2.0 & 1.9 & 1.7 & $0.195 \pm 0.006$ & Yes \\
\hline 3 & 1999 Jun 4 & 155 & $\ldots$ & $\ldots$ & 9 & 0.4 & 5 & 1 & 1.4 & 1.0 & 2.2 & $1.063 \pm 0.027$ & \\
\hline 4 & 2000 Sep 12 & 256 & 259.16 & 0.39 & 2 & 1 & 3 & 2 & 2.0 & 1.9 & 3.9 & $0.290 \pm 0.007$ & \\
\hline 5 & 2000 Oct 25 & 299 & 302.38 & 1.1 & 10 & 0.1 & 1 & 1 & 1.0 & Stat. $^{1}$ & 2.0 & $0.215 \pm 0.007$ & \\
\hline 6 & 2001 Jan 28 & 28 & 30.31 & 0.14 & 5 & 15 & 10 & 5 & 2.0 & 1.9 & 0.8 & $0.274 \pm 0.007$ & \\
\hline 7 & 2001 Apr 15 & 105 & 107.99 & 1.1 & 1 & 5 & 8 & 8 & 2.0 & 1.4 & 2.1 & $1.325 \pm 0.047$ & Yes \\
\hline 8 & 2002 Apr 21 & 111 & 113.18 & 0.27 & 6 & 1 & 10 & 30 & 2.8 & 3.9 & 19.7 & $0.752 \pm 0.043$ & \\
\hline 9 & 2002 Jul 7 & 188 & $\ldots$ & $\ldots$ & 10 & 1 & 5 & 1 & 1.4 & Stat. ${ }^{1}$ & 0.3 & $0.028 \pm 0.002$ & \\
\hline 10 & 2002 Aug 14 & 226 & $\ldots$ & $\ldots$ & 15 & Irr. $^{\mathrm{m}}$ & 5 & Irr. $^{\mathrm{m}}$ & 2.0 & Irr. $^{\mathrm{m}}$ & 2.2 & $0.253 \pm 0.009$ & Yes \\
\hline 11 & 2002 Aug 24 & 236 & 238.45 & 1.5 & 10 & 5 & Irr. $^{\mathrm{m}}$ & 10 & Irr. $^{\mathrm{m}}$ & 1.9 & 5.5 & $0.536 \pm 0.014$ & Yes \\
\hline 12 & 2003 Nov 4 & 308 & 310.79 & 0.14 & 5 & 1 & 10 & 1 & 2.8 & 1.0 & 5.2 & $1.217 \pm 0.030$ & \\
\hline 13 & $2003 \operatorname{Dec} 2$ & 336 & $\ldots$ & $\ldots$ & 25 & 6 & 10 & Irr. $^{\mathrm{m}}$ & 2.0 & Stat. ${ }^{1}$ & 3.3 & $0.887 \pm 0.022$ & Yes \\
\hline 14 & 2004 Apr 11 & 102 & 103.74 & 3.1 & 1 & 20 & 4 & 7 & 2.0 & 1.9 & 2.0 & $0.316 \pm 0.010$ & \\
\hline 15 & 2005 Jan 20 & 20 & 21.70 & 6.1 & Irr. $^{\mathrm{m}}$ & 5 & 10 & 4 & 2.0 & 1.9 & 0.7 & $0.261 \pm 0.021$ & \\
\hline 16 & 2006 Dec 13 & 347 & 348.58 & 6.1 & 0.1 & 0.5 & Irr. $^{\mathrm{m}}$ & 3 & 2.0 & 2.0 & 1.5 & $0.501 \pm 0.041$ & \\
\hline 17 & 2012 Jan 23 & 23 & 24.61 & 34 & 0.5 & 0.2 & Irr. $^{\mathrm{m}}$ & 10 & 2.0 & 2.0 & 10.5 & $0.748 \pm 0.032$ & \\
\hline
\end{tabular}

Notes.

${ }^{\text {a }}$ See Table 1.

${ }^{\mathrm{b}}$ From $\mathrm{ACE}$ and $\mathrm{SOHO}$ lists: http://www.ssg.sr.unh.edu/mag/ace/ACElists/obs_list.html and http://umtof.umd.edu/pm/shockspotter.html.

${ }^{\mathrm{c}}$ Highest energy $\left(\mathrm{MeV}\right.$ nucleon $\left.{ }^{-1}\right)$ for which $\mathrm{O}$ shows clear local maximum near shock passage time.

${ }^{\mathrm{d}}$ Decrease in $273 \mathrm{keV}$ nucleon ${ }^{-1}$ ratio during rise phase (ULEIS).

${ }^{\text {e }}$ Decrease in $12 \mathrm{MeV}$ nucleon $^{-1}$ ratio during rise phase (SIS and GOES)

${ }^{\mathrm{f}}$ Decrease in $276 \mathrm{keV}$ nucleon ${ }^{-1}$ ratio during rise phase (ULEIS).

${ }^{\mathrm{g}}$ Decrease in $\sim 12 \mathrm{MeV}$ nucleon ${ }^{-1}$ ratio during rise phase (SIS).

${ }^{\mathrm{h}} \mathrm{Using} \mathrm{Fe}$ at $276 \mathrm{keV}$ nucleon ${ }^{-1}$ as reference, scaled energy factor for $\mathrm{O}$ to give nearly time invariant ratio.

${ }^{\mathrm{i}}$ Using $\mathrm{Fe}$ at $13.2 \mathrm{MeV}$ nucleon ${ }^{-1}$ as reference, scaled energy factor for $\mathrm{O}$ to give nearly time invariant ratio.

${ }^{\mathrm{j}}$ Intersection energy in $\mathrm{MeV}$ nucleon ${ }^{-1}$ of power-law fits to low-energy and high-energy segments of $\mathrm{O}$ spectrum.

${ }^{\mathrm{k}}$ At $0.32-0.45 \mathrm{MeV}$ nucleon ${ }^{-1}$ events 1-15 from Desai et al. (2006); events 16 and 17, this work; all periods dominated by ESP intensities removed for this column.

${ }^{1}$ Statistical accuracy insufficient to compare intensities accurately.

${ }^{\mathrm{m}}$ Irregular temporal variation of $\mathrm{He} / \mathrm{H}$ or $\mathrm{Fe} / \mathrm{O}$ ratio precluded finding the ratio change or energy (if any) at which the ratio was time invariant (e.g., Figures 4(b) and (d)). 

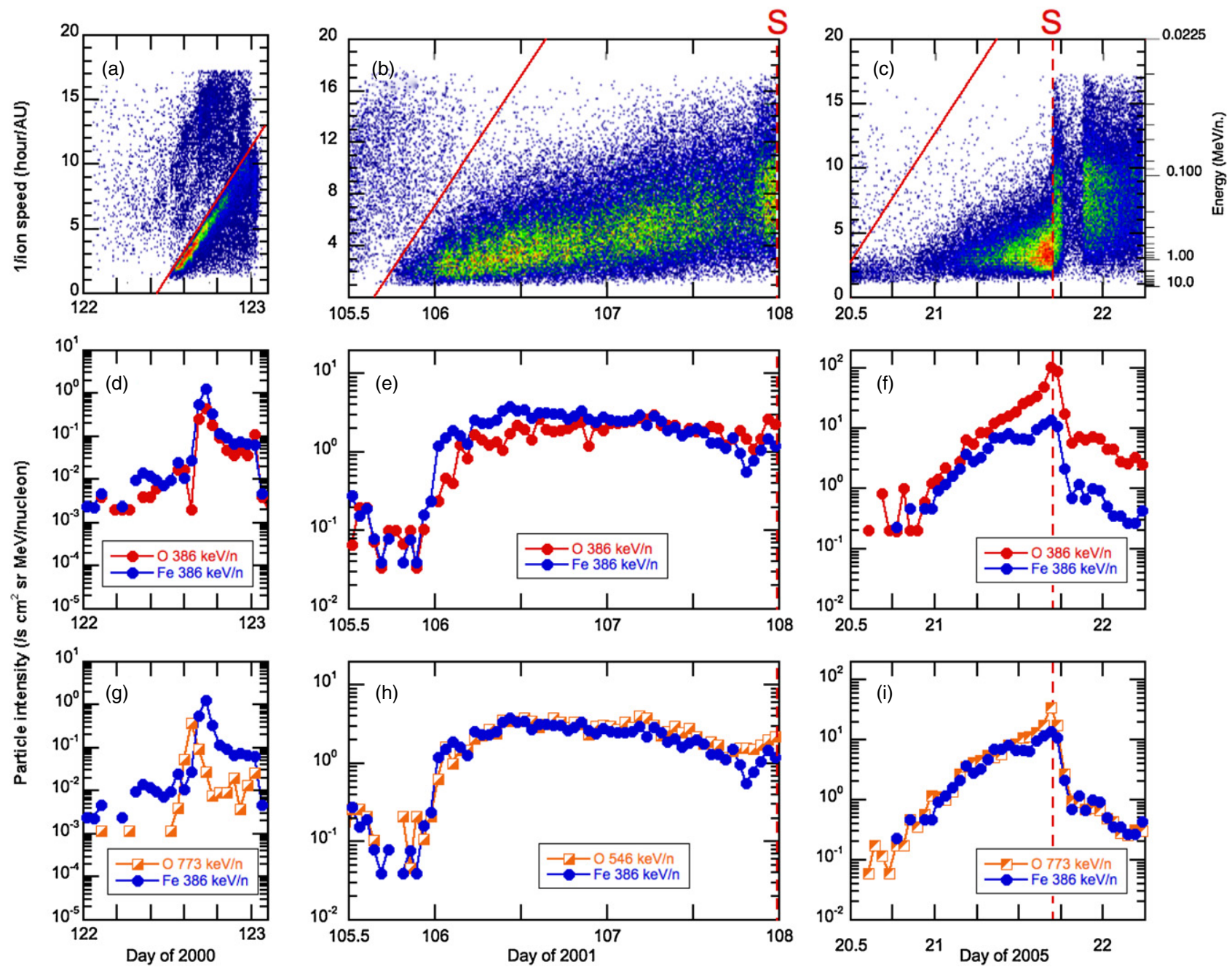

Figure 2. Columns show low energy ion data for three SEP events: left to right: 2000 May 1 (DOY 122, not included in Table 1), 2001 April 2001 (DOY 105, event 7), and 2005 January 20 (DOY 20, event 15). (a)-(c) Spectrograms for 6-80 amu ion arrivals plotted as 1/v vs. time; red diagonal lines show arrival pattern for pure velocity dispersion along a $1.2 \mathrm{AU}$ interplanetary magnetic field line for particles injected at time of X-ray flare; red dashed vertical lines marked "S" show times of shock passage; (d)-(f) $386 \mathrm{keV}$ nucleon ${ }^{-1} \mathrm{O}$ and Fe intensity profiles during the events; (g)-(i) Fe intensities at $386 \mathrm{keV}^{-1}$ nucleon $^{-1}$ repeated from middle panels compared with $\mathrm{O}$ at $773 \mathrm{keV}$ nucleon ${ }^{-1}$ (g), (i) and $546 \mathrm{keV}^{\text {nucleon }}{ }^{-1}$ (h) (see the text for discussion).

energy $\mathrm{O}$ as done in the bottom panel, the $\mathrm{O}$ profile is clearly displaced to earlier times as expected for higher energy particles (Figure 2(d) versus (g)). Events of this type occur routinely, although the example shown is perhaps the cleanest example seen so far on the $A C E$ mission; it was associated with a narrow CME (Kahler et al. 2001) and had enrichments of ${ }^{3} \mathrm{He}$ and heavy ions, but its proton intensity was too low to be included on the NOAA list of major events, and therefore is not considered in the set of events analyzed in this paper.

Contrast Figures 2(a), (d), and (g) with the middle column event in panels (b), (e), and (h) (event 7 in Table 1). The spectrogram (panel b) shows low-energy heavy ions arriving much later than would be expected from the diagonal line, as if instead of traveling 1.2 AU they had traveled many $\mathrm{AU}$ to Earth. Figure 2(e) shows that during the rise toward maximum intensity the $386 \mathrm{keV}$ nucleon ${ }^{-1} \mathrm{Fe}$ ions arrived several hours earlier than $\mathrm{O}$, and so the $\mathrm{Fe} / \mathrm{O}$ ratio decreased during the period through late day 107. Figure 2(h) shows that if we compare the middle panel $\mathrm{Fe}$ with $\mathrm{O}$ at $1.4 \times$ higher energy, the profiles nearly match leaving $\mathrm{Fe} / \mathrm{O}$ nearly constant in time (the $\mathrm{O}$ has been renormalized by a factor of 2.2).

Finally, Figures 2(c), (f), and (i) show a third type of behavior (event 15). The spectrogram for this event shows little evidence of an SEP event rise at low energies, while in fact during the period shown the event was producing a very fast-rising high energy particle population (Mewaldt et al. 2005c; Reames 2009). In contrast, the $\sim<1 \mathrm{MeV}$ nucleon ${ }^{-1}$ ions show no initial rise but rather the intensity increased gradually, peaking when the event's associated shock passed $1 \mathrm{AU}$ at $\sim 16: 45$ on day 21. Interestingly, Figure 2(f) shows that in this case the $\mathrm{Fe} / \mathrm{O}$ ratio decreases during the period, from an elevated value at the start, to a value typical of shock associated heavy ions later on. Figure 2(i) shows that if the Fe intensity is compared to the $\mathrm{O}$ intensity at twice the kinetic energy per nucleon, the differences in the $\mathrm{O}$ and $\mathrm{Fe}$ profiles are markedly reduced, although there is still a decrease in $\mathrm{Fe} / \mathrm{O}$ close to shock passage (the $\mathrm{O}$ has been renormalized by a factor of 0.5 ).

\subsection{Time-Intensity Profiles}

To illustrate the types of intensity profiles observed Figure 3 shows hourly average intensities for $\mathrm{H}, \mathrm{He}, \mathrm{O}$, and $\mathrm{Fe}$ at 4 energies from event 7 (simulation calculation lines in the figure are discussed in Section 4). At $386 \mathrm{keV}$ nucleon $^{-1}$ particle intensities rise over a period of $\sim 12 \mathrm{hr}$, increasing by one to two orders of magnitude above the pre-existing particle 

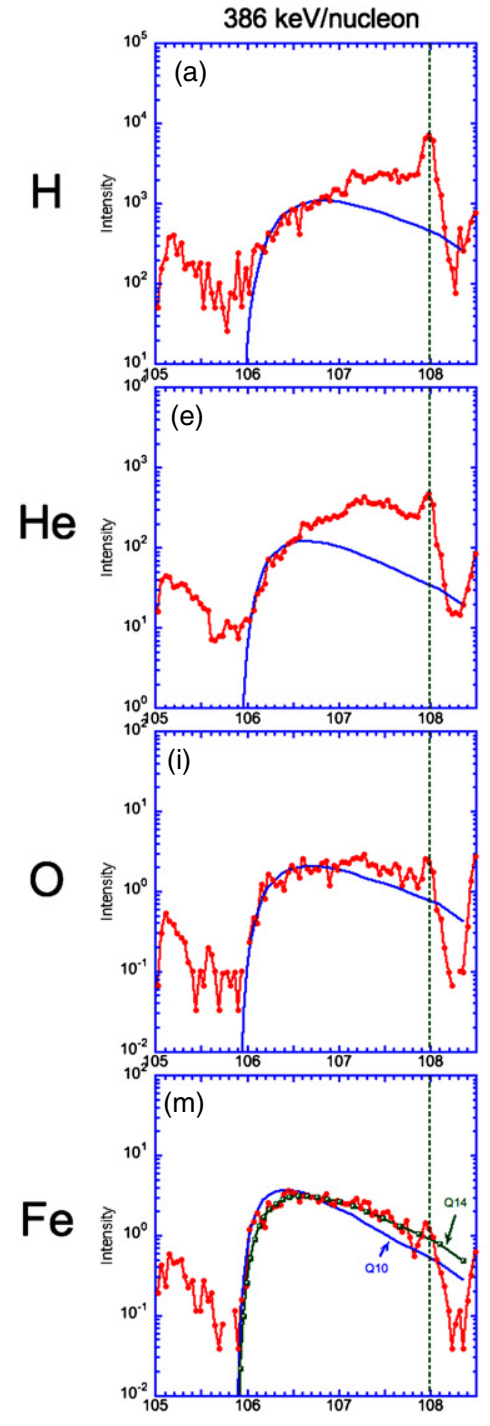
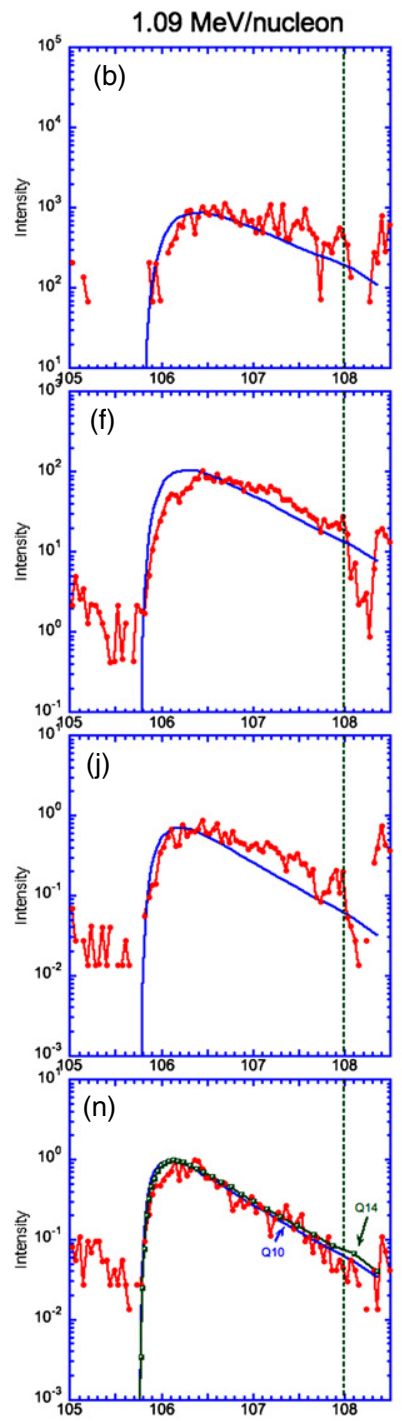
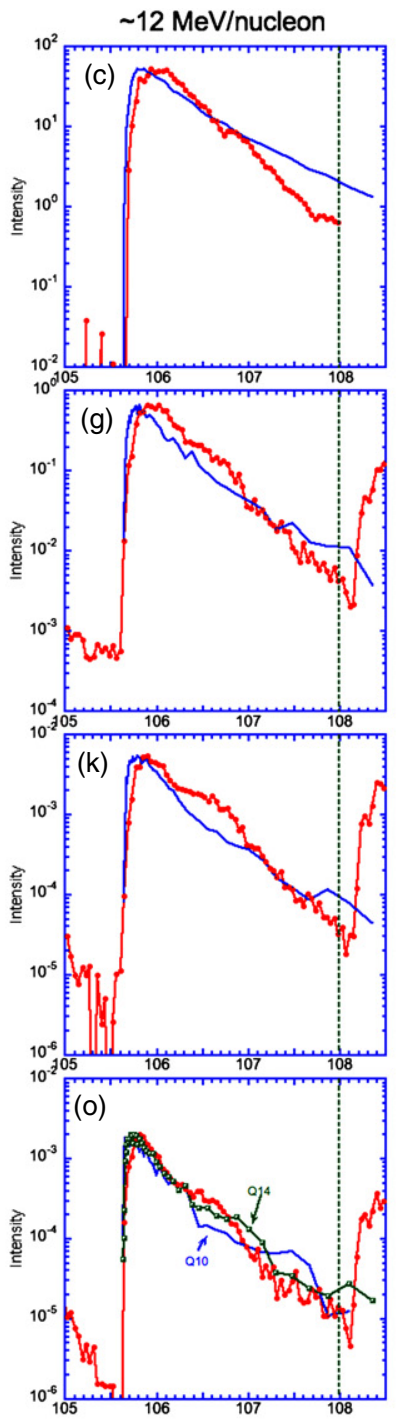
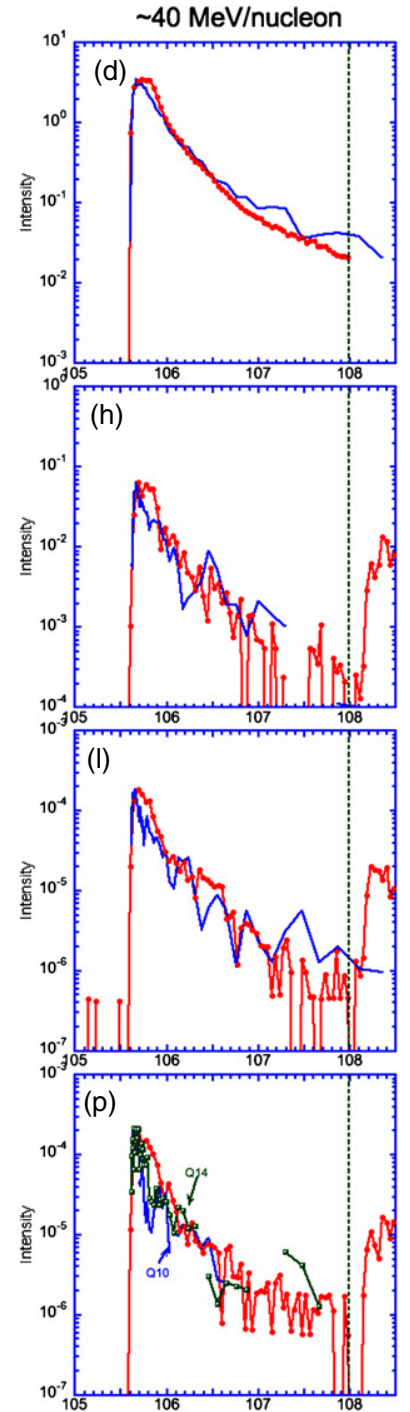

\section{Day of 2001}

Figure 3. Filled red circles: hourly average intensities of $\mathrm{H}$ (a)-(d), He (e)-(h), O (i)-(1), and Fe (m)-(p) at four representative energies for event 7 (see also Figures 2 (b), (e), (h)). Intensity units are particles/( $\left.\mathrm{s} \mathrm{cm}^{2} \mathrm{sr} \mathrm{MeV} \mathrm{nucleon}^{-1}\right)$. Particle energies for each column are listed at top. Short timescale intensity fluctuations are statistical in origin. The dashed line marks shock passage at $A C E$. The solid blue lines are simulation calculation result; the blue line for Fe shows simulation with ionization state of 10; half-filled green squares show simulation result for Fe with ionization state of 14 (see the text for details).

intensities. For $\mathrm{H}$ and $\mathrm{He}$, the interplanetary shock passage near day 108.0 influences the $386 \mathrm{keV}$ nucleon ${ }^{-1}$ intensities for a day or more before the shock arrival, with the shock passage at $A C E$ marked by a sharp, narrow increase in intensity. Notice that in contrast $\mathrm{O}$ and $\mathrm{Fe}$ at this same kinetic energy nucleon ${ }^{-1}$ show faster rises than the protons, and a much smaller intensity increase near the shock passage. For Fe (panel (m)) the shock is barely visible compared to the $\mathrm{H}$ (panel (a)) ions with the same speed, clearly showing the significant role played by the different charge-to-mass ratios of the two species. For the panels at $1.09 \mathrm{MeV}$ nucleon $^{-1}$, the effects of the shock are barely visible, and as expected the particles show earlier onset times and sharper rises than in the lower energy bin. Comparing $\mathrm{Fe}$ with $\mathrm{H}$ (panel (n) versus (b)) at this energy, the rise and decay for Fe is clearly faster. The $\sim 12 \mathrm{MeV}$ nucleon $^{-1}$ column intensity profiles (Figures 3(c), (g), (k), (o)) show fast rises with intensities reaching $\sim 50 \%$ of maximum in $2-3 \mathrm{hr}$; note, however, this rise time is much longer than the direct travel time of $\sim 1.0 \mathrm{hr}$ for a $12 \mathrm{MeV}$ nucleon $^{-1}$ particle over a $1.2 \mathrm{AU}$ flight path. The background intensities from a prior event at these energies are also comparatively much lower than at the lower energies, with the result that the SEP maximum intensities are three to four orders of magnitude above background, compared to one to two orders of magnitude at $1 \mathrm{MeV}$ nucleon $^{-1}$ and below. Finally, the $\sim 40 \mathrm{MeV}$ nucleon ${ }^{-1}$ column (Figures 3(d), (h), (l), (p)) shows faster rises, a narrower peak intensity period, and faster decays than at $\sim 12 \mathrm{MeV}$ nucleon ${ }^{-1}$. Lower statistical accuracy is apparent in the higher energy profiles. Note that for both $\sim 12$ and $\sim 40 \mathrm{MeV}$ nucleon ${ }^{-1}$ the shock passage does not affect the intensities (the increase in intensities seen later on day 108 was from a new event with X-ray maximum at 02:14).

Time-intensity profiles often show structure more complex than seen in Figures 2 and 3, especially at energies below $\sim 1 \mathrm{MeV}$ nucleon $^{-1}$. Figure 4 illustrates these differences for events 9 and 10. Event 9 was a small west-limb event that occurred during a period of low ambient intensities, and exhibited a very smooth, "classical" event profile for all species, a rare occurrence at these energies. Event 10 was associated 

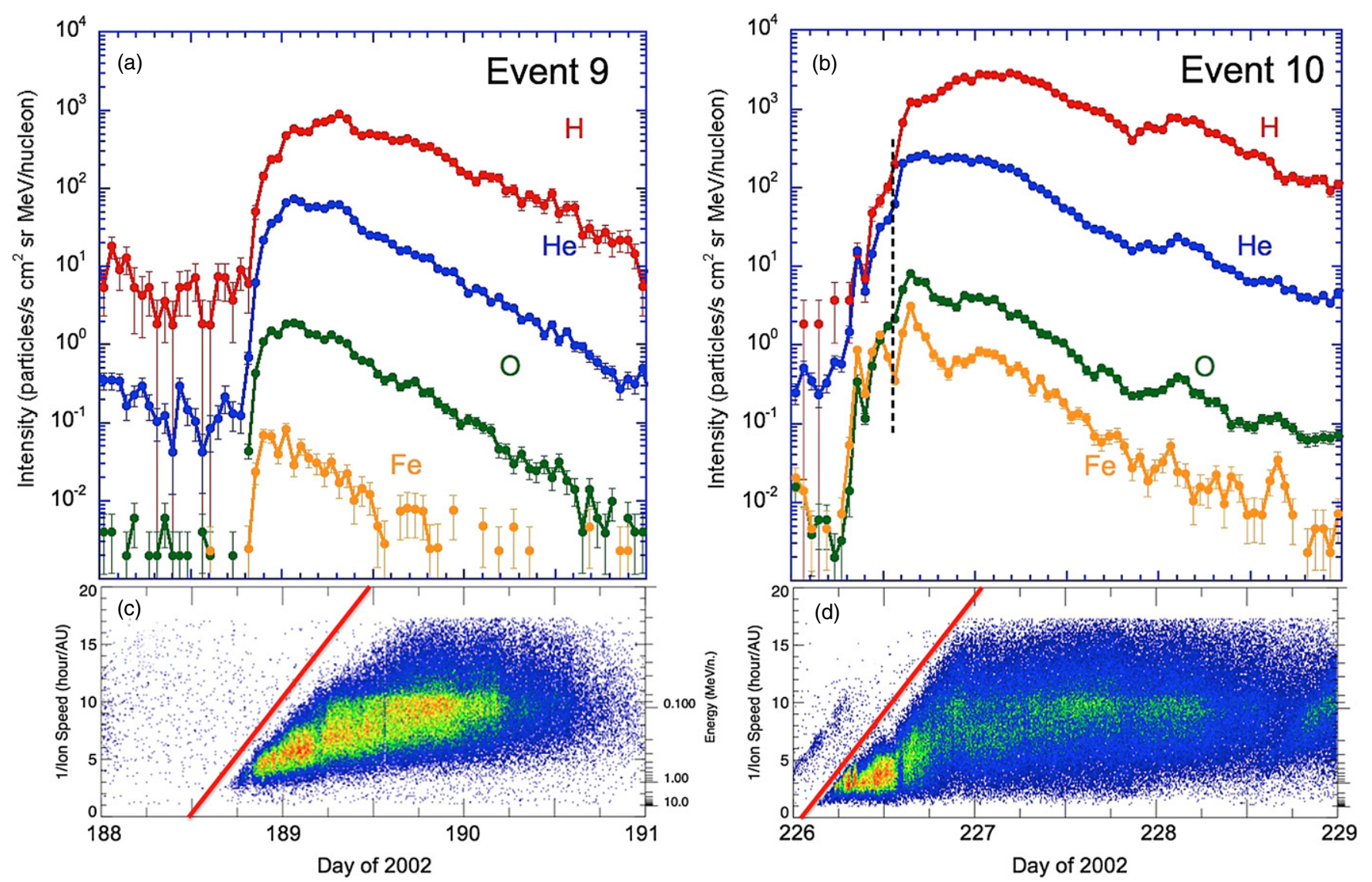

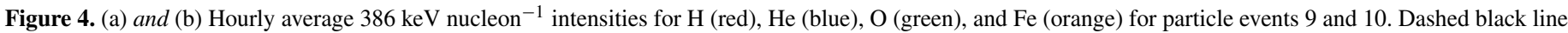
in panel (b) marks time of a change in IMF direction. (c) and (d) Particle spectrograms with red diagonal lines same as in Figure 2.

with a W54 flare and was close to ideally located for magnetic connection to Earth. Panels 4(b) and (d) show that the intensity profiles and spectrogram for event 10 show significant structure during the rise, and a more irregular decay than event 9. During the rise of event 10 (Figure 4(d)), the jaggedness in the leading edge is clearly visible and shows that particle arrivals deviated from a smooth dispersive arrival time by several hours at low energies. Figure 4(b) shows spikes in all species during the rise: higher energy particle intensity profiles show these spikes at the same times (Figure 4(d)), so they are consistent with being spatial features. The dashed line around day 226.53 marks a time when the interplanetary magnetic field (IMF) changed direction by $\sim 30^{\circ}$ for about one-half hour. This coincides with a large dip in the Fe intensity and a change in the rise rate of the $\mathrm{H}$, $\mathrm{He}$, and O. Although it would take observations from many spacecraft to fully understand the structures responsible for this behavior, we ascribe them to anisotropies and/or changing magnetic connection to the acceleration region. These cause the $A C E$ instruments to sample different portions of the pitch angle distribution, or regions of the accelerating region that have different though closely related intensities (for discussions of these effects in SEP events observed with multiple spacecraft, see von Rosenvinge et al. 2009; Chollet et al. 2010b). Intensity changes of this type have been observed in both electrons and ions and although they are sometimes correlated with changes in the local IMF, often that is not the case (Anderson \& Dougherty 1986; Mazur et al. 2000; Chollet \& Giacalone 2008). At higher energies irregularities in the event profiles are less distinct (e.g., Figure 3) probably due to a lesser sensitivity to the magnetic field changes, and a faster timescale overall.
There is an additional effect due to the long timescales of low energy particle intensity profiles, wherein the Sun's $13^{\circ}$ day $^{-1}$ rotation can also lead to significantly different connections to the acceleration region during the course of an SEP event, thereby affecting the intensities during the decay phase (McCracken \& Rao 1970).

Anticipating our model fitting described below, we note that the propagation model we use essentially describes particle motion in a single flux tube and cannot fit fine structure such as that shown for event 10 . In cases like this, the smooth simulation profiles were judged adequate if they formed a reasonable envelope to the jaggedness; if the deviations were too extreme, no fit was made.

\subsection{Differential Energy Spectra}

As an example of typical spectra, Figure 5 shows event 7 fluences for $\mathrm{H}, \mathrm{He}, \mathrm{O}$, and $\mathrm{Fe}$ summed from 2001 April 15 12:00 through April 18 00:00 (DOY 105-108). The spectra are typical for large SEP events, with steeper high-energy portions gently rolling over toward harder values at low energies: for $\mathrm{H}, \mathrm{He}, \mathrm{O}$, and $\mathrm{Fe}$, respectively, between 0.15 and $1.0 \mathrm{MeV}$ nucleon $^{-1}$ the power-law slopes are 1.12, 1.06, 1.10, and 1.32. Between 10 and $100 \mathrm{MeV}$ nucleon $^{-1}$ the respective slopes for $\mathrm{H}, \mathrm{He}$, and $\mathrm{O}$ are 2.06, 2.78, and 2.70, and between 2 and $100 \mathrm{MeV}$ nucleon ${ }^{-1}$ the slope for Fe is 2.51 (see also Tylka et al. 2002). Because of the gentle rollovers and sparse coverage in some ranges, we cannot locate the spectral breaks precisely in Figure 5; however, inspection of the figure indicates that the Fe break is near $1 \mathrm{MeV}$ nucleon $^{-1}$, and for lower mass particles, the break 


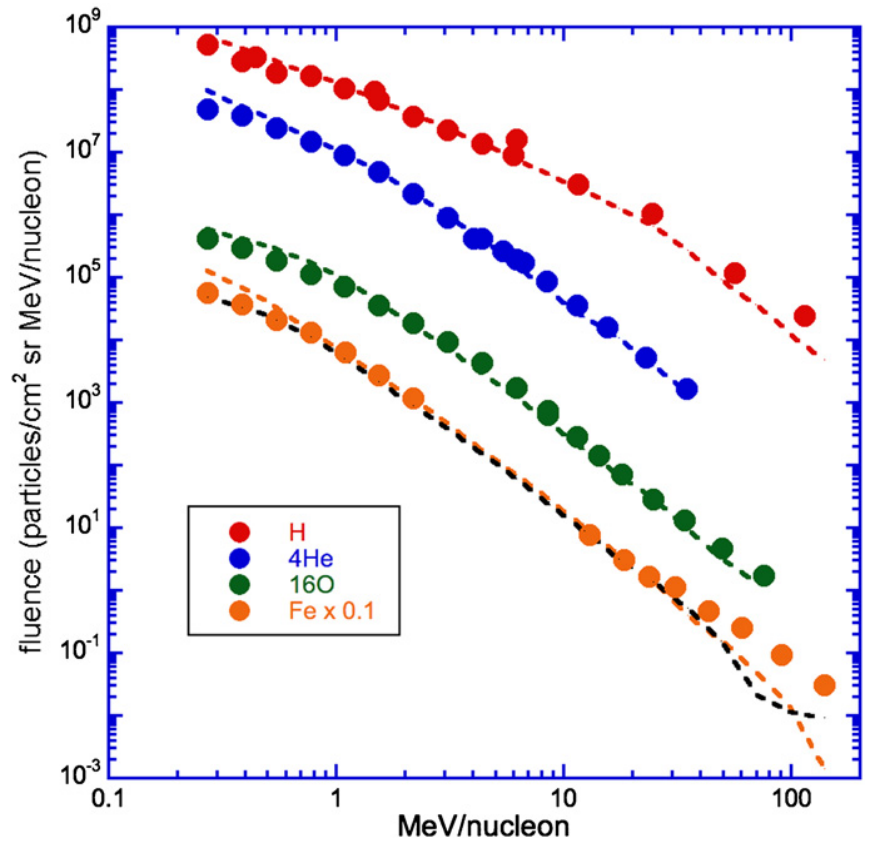

Figure 5. Filled circles: differential fluence spectra for $\mathrm{H}, \mathrm{He}, \mathrm{O}$, and $\mathrm{Fe}$ summed from 2001 April 15 12:00 to April 18 00:00 (DOY 105-108, event 7); error bars are smaller than the points. Dashed lines are simulation calculation intensities summed over the event. The dashed brown line is Fe assuming a charge state of 10; dashed orange line is for Fe assuming a charge state of 14 (see the text). Below $\sim 1 \mathrm{MeV}^{\text {nucleon }}{ }^{-1}$ the $\mathrm{H}$ and $\mathrm{He}$ spectra are dominated by the shock passage (compare Figure 3(a) and (e)).

is at higher energies. This is qualitatively consistent with the previously observed trend for break energies to occur at lower energy per nucleon with increasing particle mass (Mewaldt et al. 2005b; Li et al. 2009b).

\subsection{Fe/O and He/H Temporal Variations}

Figure 6 shows the hourly average $\mathrm{Fe} / \mathrm{O}$ ratio for all the events at $276 \mathrm{keV}$ nucleon $^{-1}$ (red points), and $\sim 12 \mathrm{MeV}$ nucleon $^{-1}$ (blue points). As seen in the figure and listed in Table 2, decreases in $\mathrm{Fe} / \mathrm{O}$ associated with the rise phase are present in 13 of 17 events at $276 \mathrm{keV}$ nucleon $^{-1}$, and 11 of 17 events at $\sim 12 \mathrm{MeV}$ nucleon $^{-1}$. Not all events fit this pattern, for example event 5 was a well-connected event (W50; Kahler 2005) without visible shock involvement that had enriched ${ }^{3} \mathrm{He} /{ }^{4} \mathrm{He}(\sim 1 \%)$, but had no visible time dependence in the $\mathrm{Fe} / \mathrm{O}$ at low energies, while at higher energies the $\mathrm{Fe} / \mathrm{O}$ change, if any, was small. Rise-phase-related changes in $\mathrm{He} / \mathrm{H}$ are also listed in the table and shown in Figure 7. Since $(Q / M)_{\mathrm{He}} /(Q / M)_{\mathrm{H}} \sim(Q / M)_{\mathrm{Fe}} /(Q / M)_{\mathrm{O}}$ it is reasonable to expect similar behavior for the $\mathrm{He} / \mathrm{H}$ and $\mathrm{Fe} / \mathrm{O}$ ratios during the SEP onset phase. However, there are clear differences: decreases in $\mathrm{He} / \mathrm{H}$ associated with the rise phase are seen in 11 of 17 events at $276 \mathrm{keV}$ nucleon $^{-1}$, and 7 of 17 events at $\sim 12 \mathrm{MeV}$ nucleon $^{-1}$. This is a significantly lower fraction than for $\mathrm{Fe} / \mathrm{O}$. In addition, there are cases (e.g., events 16, 17) where $\mathrm{He} / \mathrm{H}$ increases with time during the rise phase, a behavior not seen for $\mathrm{Fe} / \mathrm{O}$. This contrast in behavior between $\mathrm{He} / \mathrm{H}$ and $\mathrm{Fe} / \mathrm{O}$ has been reported previously (Witte et al. 1979; Mason et al. 1983; Reames et al. 2000).

\section{INTERPLANETARY TRANSPORT MODEL}

We now seek to model these observations using a more complete picture than our earlier study, which used only simple arguments based on the diffusion coefficient. In a large SEP event, several distinct diffusive processes are entangled. These include (a) transport in the interplanetary medium, (b) acceleration processes at a shock in the inner heliosphere, or (c) release of particles from an acceleration region at the Sun or associated with a shock. In this paper, we explore the extent to which our observations can be explained by a detailed treatment of the transport process including pitch angle scattering, focusing, and adiabatic cooling. We assume that energetic particles are released close to the Sun at $0.0547 \mathrm{AU}$ and follow these particles all the way to $1 \mathrm{AU}$. Therefore, we only consider the processes involved in transport in the interplanetary medium. An alternate approach could be to use a model that includes an interplanetary shock and/or growth of interplanetary waves such as was done by some authors of this paper and others (Ng \& Reames 1994; Ng et al. 1999, 2003; Zank et al. 2000; Rice et al. 2003; Li et al. 2005; Verkhoglyadova et al. 2010 and references therein). In order to include the treatment of a moving shockwave or particle-wave interactions at the shock, such approaches will introduce many new, and not well-constrained parameters into the fitting. Furthermore, some of these approaches $(\mathrm{Ng} \&$ Reames 1994; $\mathrm{Ng}$ et al. 1999) necessarily restrict the treatment of transport due to computational limitations. As an example of the practical tradeoffs required in all these studies, the investigation by $\mathrm{Ng}$ et al. (1999) analyzed Fe/O temporal variations of the type studied here, and while their model included shock wave growth, its simplifying assumptions included a radial solar wind magnetic field and no adiabatic deceleration. With our focus on the role of interplanetary propagation in the observational properties presented above, we seek to help separate source from transport effects. Although it will be seen that our transport model fits a large portion of the observations, some data (especially at low energies) are not fitted, giving evidence for a significant role played by acceleration and release effects from shocks or other processes. By limiting our choice of SEP events to the western hemisphere we minimize the effects of shocks in the events studied here. We note that the diffusive transport picture and method of calculating adiabatic deceleration used by us and others cited above is not universally accepted (e.g., Roelof 2000).

The approach for each SEP event will be to impulsively release spectra of $\mathrm{H}, \mathrm{He}, \mathrm{O}$, and $\mathrm{Fe}$, and adjust interplanetary turbulence parameters to seek a fit to the observed intensity profiles. By simultaneously fitting species with different charge to mass ratios over a much broader energy range than prior work, we seek to constrain the fits and derive propagation parameters broadly representative of conditions relevant to $1 \mathrm{AU}$ observations.

\subsection{Propagation Model}

The interplanetary transport model used here is based on the Boltzman-Vlasov equation (Skilling 1971; Earl 1974, 1976; Ruffolo 1995; Isenberg 1997; Kocharov et al. 1998; Lario et al. 1998; Zhang 1999; Kota et al. 2005; Aran et al. 2006; Qin et al. 2006; le Roux \& Webb 2007; Aran et al. 2008; Zhang et al. 2009; Chollet et al. 2010a; Dröge et al. 2010). In this work, we cast the focused transport equation into a set of equations describing the motion of individual particles. The first-order terms are followed using the method of characteristics (e.g., Sarra 2003). The second-order term is followed using a Monte Carlo technique (e.g., Zhang 1999; Li et al. 2003, 2005, and references therein). Mathematically, our approach is equivalent 

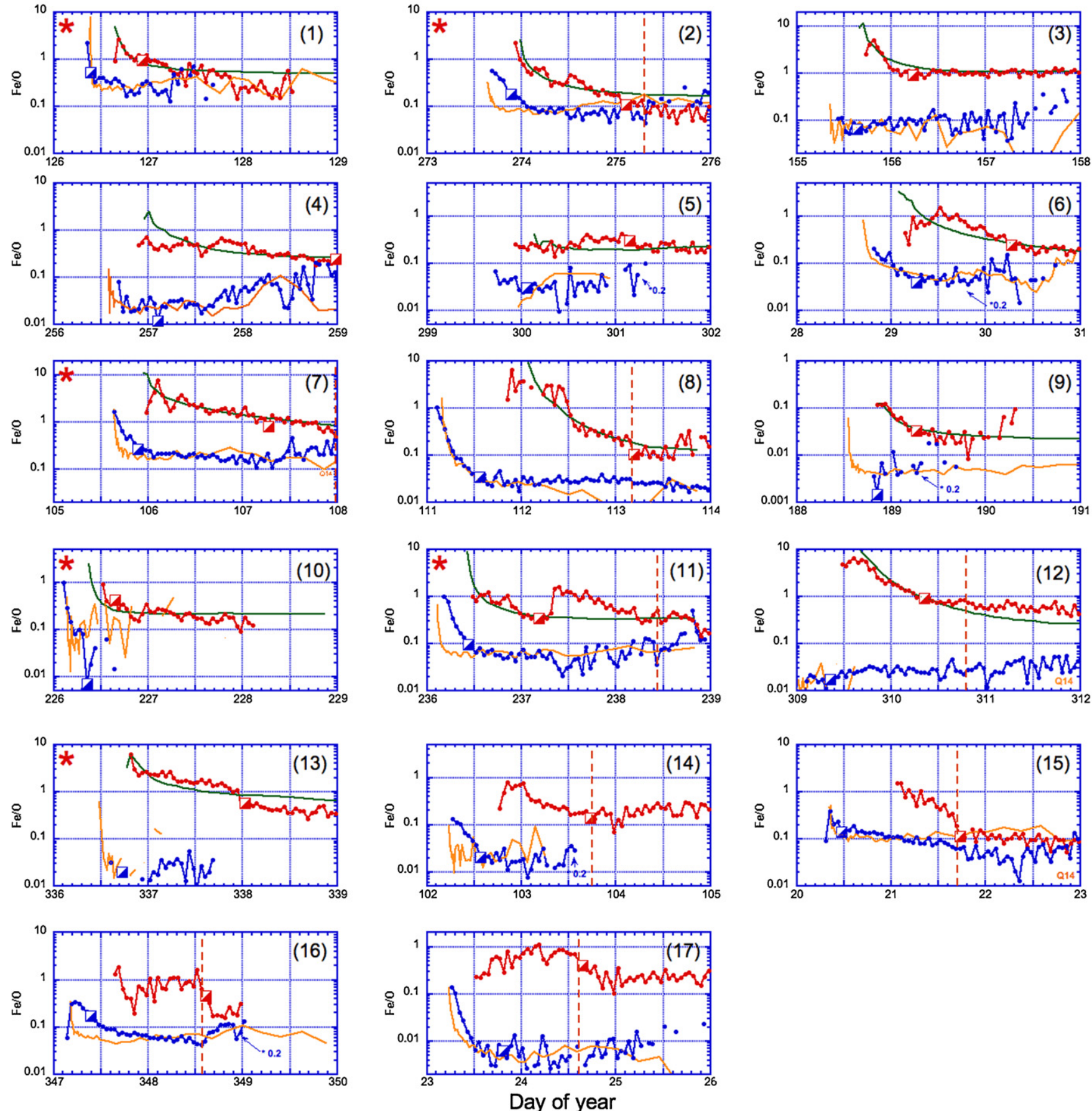

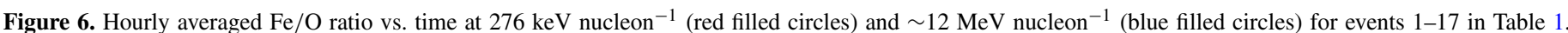

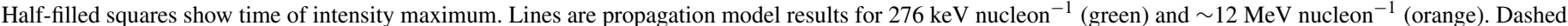

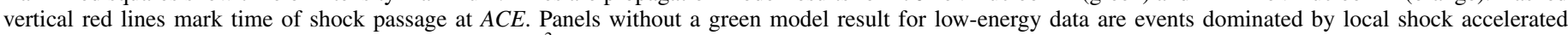
particles. Red asterisks denote events with clear ambient ${ }^{3} \mathrm{He}$ - and Fe-rich energetic particles preceding the event.

to using a stochastic differential equation method to solve a forward Kolmogorov equation. Physically, the first-order terms describe the deterministic part of a single charged particle's motion in a background magnetic field $B_{0}$ (Parker spiral in this case), the second-order term describes diffusion of particle pitch angle due to the turbulent magnetic field $\delta B$ in the solar wind, and is stochastic in nature. In following the pitch angle scattering, we follow $\mathrm{Li}$ (2008) who replaced the scattering mean free path with a description using the phase space diffusion coefficient $D_{\mu \mu}$ to include pitch angle scattering in small increments (see also Qin et al. 2005). Using $D_{\mu \mu}$ instead of the mean free path is desirable since in some SEP events the inferred mean free path is larger than 1 AU. In such cases, the mean free path does not capture the evolution of the particle pitch angle during propagation.

Previous research has shown that adiabatic deceleration significantly speeds the decay rates of low-energy ions (e.g., Ruffolo 1995; Kocharov et al. 1998; Zhang et al. 2009) and we found it necessary to include this effect for the data here, which extends to considerably lower energies than the cited work. The 

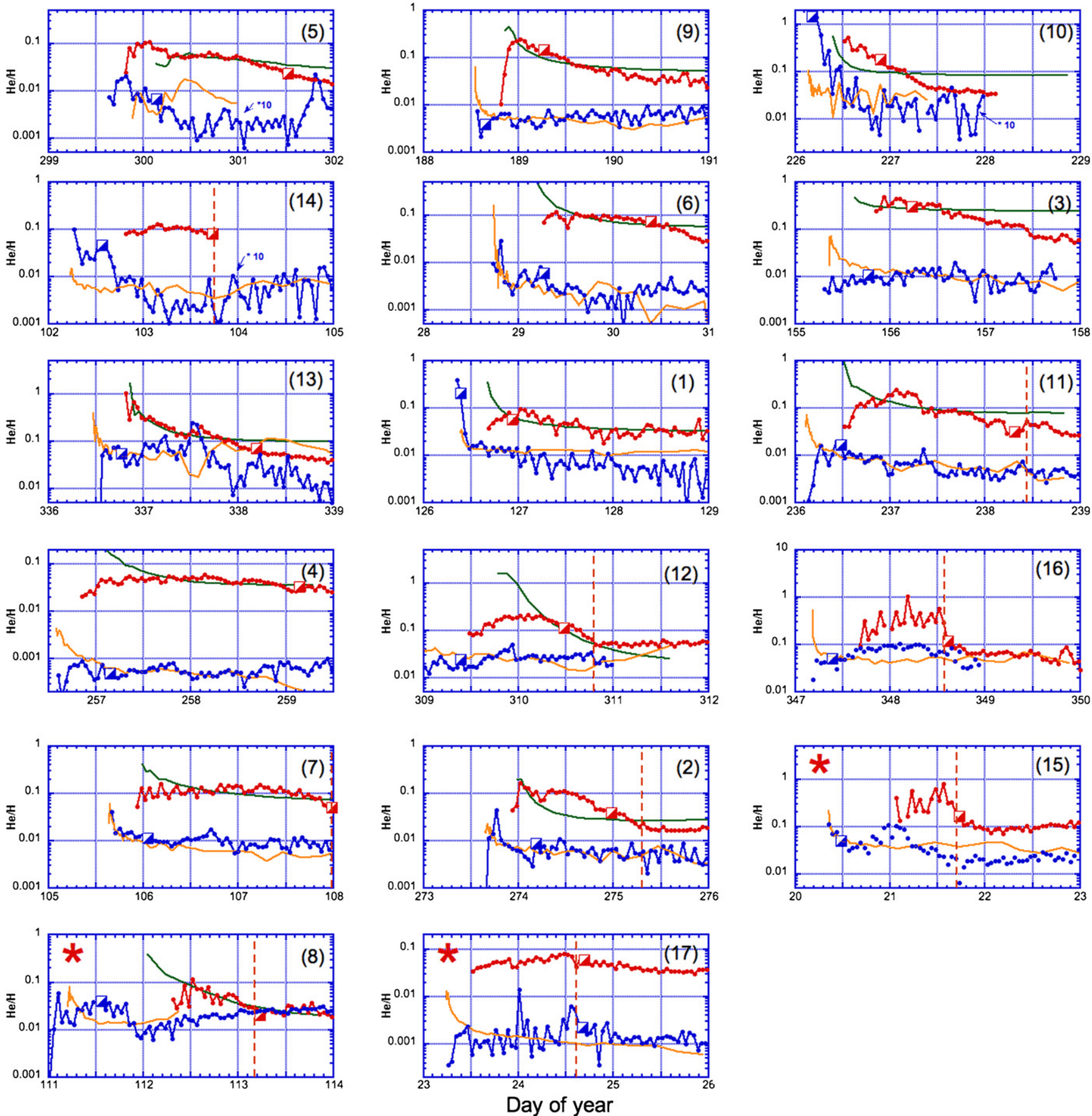

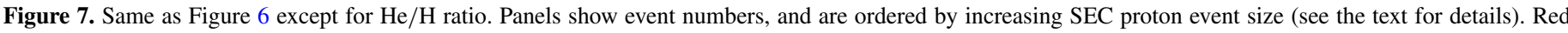
asterisks mark events with peak proton intensity exceeding the event modeled for proton-amplified waves by Tylka et al. (1999).

adiabatic deceleration term is based on Parker (1965), modified to avoid the assumption of weak anisotropy (Webb \& Gleeson 1979; Ruffolo 1995):

$$
\frac{d p^{\prime}}{d t}=-p^{\prime} \frac{U}{r}\left\{\mu^{\prime 2} \sin ^{2} \Phi+\frac{1-\mu^{\prime 2}}{2}\left(1+\cos ^{2} \Phi\right)\right\}
$$

where $p^{\prime}$ is particle momentum in the solar wind, $\mu$ is the pitch angle cosine in the solar wind frame, $U$ is the solar wind speed, and $\Phi$ is the angle between $B$ and $\boldsymbol{r}$. We assume that the Earth is in the ecliptic plane and that the magnetic field geometry is an Archimedes spiral. The adiabatic deceleration term completes the deterministic portion of the particle motion.
We now turn to the evolution of $\mu$ during the particle transport due to pitch angle scattering. This term has two parts, where the first describes the $\mu$ dependence of $D_{\mu \mu}$ and is given by

$$
d \mu=\frac{\partial D_{\mu \mu}}{\partial \mu} d t .
$$

Note that this term is of first order in time and can be evaluated in the same way as the deterministic change of $\mu$ due to focusing. The second-order term is the random walk in $\mu$ and is handled in a stochastic manner described in the following section.

To relate $D_{\mu \mu}$ to the interplanetary turbulence we follow Bieber et al.'s (1994) modification of the Quasi-linear Theory 
treatment by Jokipii $(1966,1971)$. For the ion energies in this study, we are outside the dissipation range in interplanetary turbulence, and in this case

$$
D_{\mu \mu}=\frac{2 \pi C}{B_{0}^{2}} \frac{v}{R_{L}}\left(\frac{R_{L}}{\lambda_{C}}\right)^{q_{i}-1},
$$

where the $C$ and $\lambda_{C}$ are the turbulence strength and correlation length, $v$ is the particle velocity, $B_{0}$ is the Parker magnetic field, $R_{L}$ is the particle's Larmor radius, and $q_{i}$ is the power spectral index of the turbulence. At $1 \mathrm{AU}$, we follow Bieber's values: $C=0.5 \mathrm{nT}^{2}$ and $\lambda_{C}=4.55 \times 10^{6} \mathrm{~km}$, and $q_{i}=5 / 3$, which reproduce several observational studies. Note that the dependence of $D_{\mu \mu}$ on the turbulent magnetic field strength implies that $D_{\mu \mu}$ will vary with $r$. How $\delta B$ varies with $r$ is presently unknown, although some earlier studies by Helios (see review by Bruno \& Carbone 2005) suggested that a WKB approximation, i.e., $\delta B^{2} \sim r^{-3}$ is reasonable, but a slight underestimate. In this work, we assume that the turbulence level has an $r^{-3.5}$ dependence. Thus, scattering is stronger close to the Sun, but this is partly offset by stronger magnetic focusing.

To compare the present calculations with previous work, we derive the particle's parallel mean free path with the standard expression (Jokipii 1966; Hasslemann \& Wibberenz 1968; Earl 1974):

$$
\lambda=\frac{3}{4} v \int_{0}^{1} d \mu \frac{\left(1-\mu^{2}\right)^{2}}{D_{\mu \mu}}
$$

\subsection{Monte Carlo simulation}

With the deterministic portion in place, particles are released close to the Sun with an initial isotropic velocity distribution in the outward-facing hemisphere in phase space. The initial particle energies are chosen so that a specified spectrum will emerge, with power-law segments $f(E) \sim E^{-\gamma}$ and an adjustable energy where the segments meet (spectral "break"). At each time step, the particle pitch angle, location, and momentum are updated for the deterministic component, and the particle scattering is updated for the stochastic component using random Gaussian sampling, where the change in pitch angle is given by

$$
\tilde{d} \mu=\mu(t+d t)-\mu(t)=\operatorname{sign}\left(\xi^{\prime}-\frac{1}{2}\right) \operatorname{erf}^{-1}(\xi) \sqrt{4 D_{\mu \mu} d t}
$$

where $\xi$ and $\xi$, are two uniform random numbers between 0 and 1 and $\operatorname{erf}^{-1}$ is the inverse error function. With this selection the random $\tilde{d} \mu$ will follow a Gaussian distribution with zero mean and standard deviation $\sqrt{2 D_{\mu \mu} d t}$. In evaluating Equation (6) it is necessary to keep the time steps small so that the scattering changes remain small throughout. The maximum time step in the simulation is taken as $d t=(1 /$ iNstep $) *(1 a u / v)$, where iNstep $=12,000$ for a typical run gives $d t=3.9 \times 10^{-5}$ and $2.6 \times 10^{-4} \mathrm{~s}$ for 2 and $100 \mathrm{MeV}$ nucleon ${ }^{-1}$, respectively, with corresponding pitch angle cosine changes of $d \mu=0.02$ and 0.05 from focusing. In the code we also require the time step to be small so that $\tilde{d} \mu$ from Equation (6) never exceeds 0.2 . In our code, the propagation of particles along the Parker field line is modeled in an instantaneous inertial frame that is co-rotating with the Sun and the diffusion of pitch angle is followed in the solar wind frame. At each time step, frame transformations between the solar wind frame and the instantaneous inertial frame are performed.
Since particle mass-to-charge ratio enters the scattering coefficient through the Larmor radius term in Equation (4), each element with different mass-to-charge ratio requires a separate calculation. Typically, $\sim 10^{6}$ particles were injected ${ }^{6}$ and each particle followed continuously up to a time limit (typically $60 \mathrm{hr}$ ). There was no outer boundary; however, if particles reached the solar surface they were absorbed. Each time the particle crossed 1 AU (either outward- or inward-moving) the following quantities were saved: time since injection, pitch angle, speed, radial distance, speed at injection, and pitch angle at injection. Since each particle is treated independently, the calculation is highly suitable for parallel processing. Observable quantities such as particle intensity, spectra, and pitch angle distributions were obtained by summing over the $1 \mathrm{AU}$ crossings data.

\subsection{Example Calculation}

We now show a sample simulation calculation output for $\mathrm{O}$ and $\mathrm{Fe}$ to illustrate the types of solutions and the effect of different charge-to-mass ratios. We assumed a constant solar wind speed of $420 \mathrm{~km} \mathrm{~s}^{-1}$ and a magnetic field of $B=1.53 \mathrm{G}$ at the surface of the Sun. Then, input parameters for both species were specified as shown in Table 3. The spectral indices and relative break energies were typical of the events fitted; however, for simplicity in interpreting the results, we chose identical spectral slopes for the two species, whereas usually the Fe spectrum is somewhat steeper than $\mathrm{O}$. The ionization states were chosen to be typical of the slow solar wind (von Steiger et al. 2000), and the values of $D_{\mu \mu}$ and $q_{i}$ were chosen to yield a turbulence spectrum similar to Bieber et al. (1994). Figure 8 shows the typical interplanetary turbulence spectrum from Bieber et al. (1994) based on the survey of Wanner \& Wibberenz (1991b) along with line segments of different colors showing turbulence levels for some of the events in Table 1 over the wavenumber range of the fits. The $D_{\mu \mu}$ and $q_{i}$ values in Table 3 are typical of the events fitted below, and the equivalent turbulence level is indicated by line number 7 in the plot (slightly offset to distinguish it from the red line). In terms of the particle scattering mean free path, this level is equivalent to $\sim 0.1-0.2 \mathrm{AU}$ for low-energy heavy ions at 1 AU.

Figure 9 shows output from the sample run for $\mathrm{O}$ nuclei injected at the time of event 7, where each particle is tallied in the spectrogram each time it crosses $1 \mathrm{AU}$. The spectrogram is qualitatively similar to the spectrogram for event 7 , as shown in Figure 2(b). Note that the peak of the particle distribution follows a smooth line at an angle that indicates transport much slower than pure velocity dispersion, which would give a distribution parallel to the slanted line in the panel. This pattern is typical of events where there is no particle intensity increase associated with a shock passage; for events and energies where the shock passage dominates the intensity profiles (e.g., Figure 2(c)) there is a clear difference in appearance wherein few particles are seen at first and then there is a comparatively sudden rise in intensity when the shock passes. (To facilitate comparing with Figure 2(b) the plot in Figure 9 simulated the decrease in geometry factor of ULEIS below $\sim 80 \mathrm{keV}^{\text {nucleon }}{ }^{-1}$ )

Figure 10 shows sample-calculated time-intensity profiles. Filled red circles show typical intensity profiles qualitatively similar to the different energy panels in Figure 3, namely

\footnotetext{
6 In this paper, injection refers to release of a fully accelerated particle population into the IMF flux tube near the Sun, not to injection of a seed population into a region where particle acceleration takes place.
} 
Table 3

Sample Run Parameters

\begin{tabular}{|c|c|c|c|c|}
\hline Species/Quantity & \multicolumn{2}{|c|}{ Oxygen } & \multicolumn{2}{|c|}{ Iron } \\
\hline Species $M, Q$ & \multicolumn{2}{|c|}{16,6} & \multicolumn{2}{|c|}{56,10} \\
\hline \multirow[t]{2}{*}{ Energy range of injected particles $\left(\mathrm{MeV}\right.$ nucleon $\left.^{-1}\right)$} & \multicolumn{2}{|c|}{$0.05-200$} & \multicolumn{2}{|c|}{$0.05-200$} \\
\hline & Injected & at $1 \mathrm{AU}$ & Injected & at $1 \mathrm{AU}$ \\
\hline Low-energy power-law index ${ }^{\mathrm{a}}$ & 2.0 & 1.36 & 2.0 & 1.41 \\
\hline High-energy power-law index ${ }^{a}$ & 4.0 & 3.60 & 4.0 & 3.64 \\
\hline Spectral break energy $\left(\mathrm{MeV}\right.$ nucleon $\left.^{-1}\right)$ & 4.0 & 2.53 & 2.0 & 1.29 \\
\hline Injection radius (AU) & \multicolumn{2}{|c|}{0.0547} & \multicolumn{2}{|c|}{0.0547} \\
\hline Injection radius (solar radii) & \multicolumn{2}{|c|}{11.8} & \multicolumn{2}{|c|}{11.8} \\
\hline$D_{\mu \mu}$ at $1 \mathrm{AU}(\mathrm{Hz})$ & \multicolumn{2}{|c|}{4.0} & \multicolumn{2}{|c|}{4.0} \\
\hline$D_{\mu \mu}$ radial dependence & \multicolumn{2}{|c|}{-0.83} & \multicolumn{2}{|c|}{-0.83} \\
\hline$q_{i}$ & \multicolumn{2}{|c|}{$\begin{array}{l}-0.83 \\
1.667\end{array}$} & \multicolumn{2}{|c|}{1.667} \\
\hline Number of injected particles (all energies) & \multicolumn{2}{|c|}{$2.22 \times 10^{6}$} & \multicolumn{2}{|c|}{$2.21 \times 10^{6}$} \\
\hline Number of $1 \mathrm{AU}$ crossings (all energies) & \multicolumn{2}{|c|}{$3.75 \times 10^{6}$} & \multicolumn{2}{|c|}{$3.73 \times 10^{6}$} \\
\hline \multicolumn{5}{|l|}{ Number of $1 \mathrm{AU}$ crossings per particle } \\
\hline $0.386 \mathrm{MeV}$ nucleon $^{-1}$ & \multicolumn{2}{|c|}{2.58} & \multicolumn{2}{|c|}{2.63} \\
\hline $5.12 \mathrm{MeV}$ nucleon $^{-1}$ & \multicolumn{2}{|c|}{6.23} & \multicolumn{2}{|c|}{5.42} \\
\hline $20.48 \mathrm{MeV}$ nucleon $^{-1}$ & \multicolumn{2}{|c|}{6.7} & \multicolumn{2}{|c|}{5.6} \\
\hline
\end{tabular}

Note. ${ }^{\mathrm{a}} \gamma$ for differential energy spectra of the form $d J / d E=A E^{-\gamma}$ where $A$ is a constant.

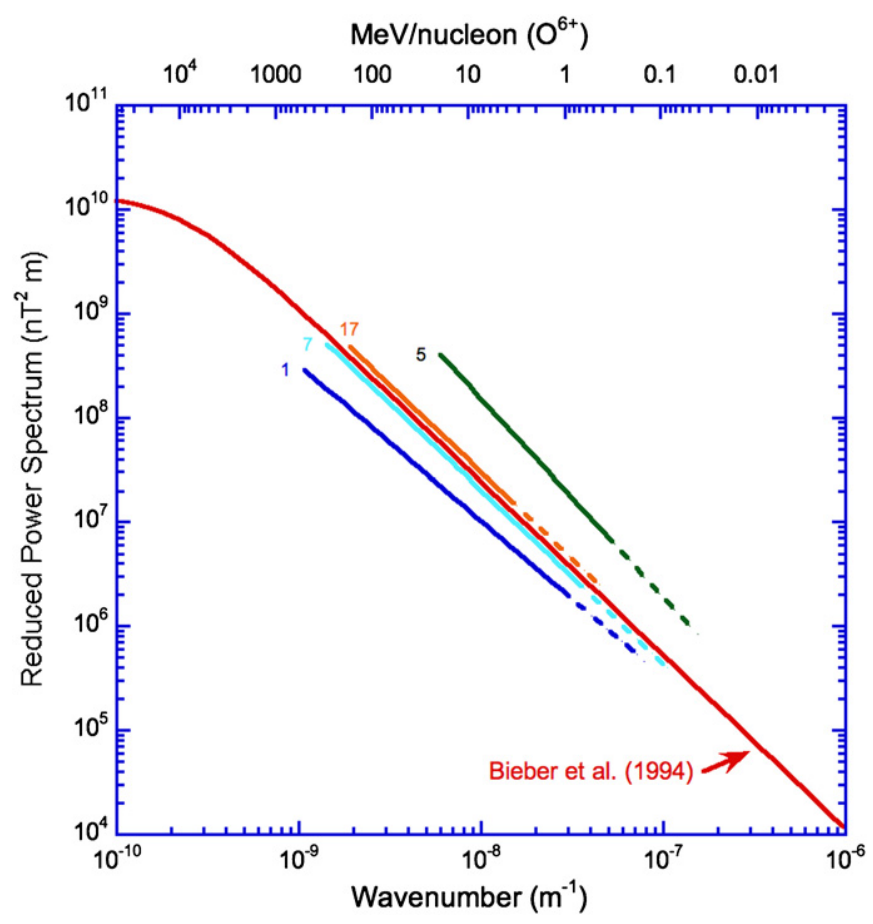

Figure 8. Red line: interplanetary turbulence spectrum $q^{-5 / 3}$ at 1 AU from Bieber et al. (1994). Numbered solid lines show turbulence levels for representative fits to the numbered events in Table 1. Solid lines show the wavenumber range for the ion fits for typical pitch angles, and dashed extensions shown region probed by particles with pitch angles a factor of 10 smaller. The transition to the "dissipation range" change of slope to $\sim q^{-3}$ occurs above wavenumbers of $10^{-5}$ (Jokipii 1971), well beyond the range of this plot (e.g., Bieber et al. 1994). The scale for $\mathrm{O}^{6+}$ at the top is for the approximation that wavenumber $\sim 1$ /gyroradius (e.g., Jokipii 1971).

slower rises and decays at lower energies, with faster rise and shorter peak duration at high energy. The time bins in the figure are of systematically increasing width to better show the details of the rise phase. The other solid lines in the figure show the original injected energy of the particles counted at
1 AU in the energy bins shown, and their sum is shown with the filled red circle trace. Figure 10(a) shows that at $0.32-0.45 \mathrm{MeV}$ nucleon $^{-1}$, adiabatic deceleration has a very strong effect: indeed virtually none of the particles observed in this energy range at $1 \mathrm{AU}$ began in the same energy bin at injection. At the peak of the event, the particles come from energies $\sim$ twice that observed at $1 \mathrm{AU}$. As time goes on and the particles continue to lose energy, the $0.32-0.45 \mathrm{MeV}_{\text {nucleon }}{ }^{-1}$ bin at $1 \mathrm{AU}$ is filled with particles whose injected energies were increasingly higher, until by $80 \mathrm{hr}$ following injection the particles seen at $1 \mathrm{AU}$ originally had energies in the range 1.28-2.56 $\mathrm{MeV}$ nucleon $^{-1}$. Figure 10(b) shows the same quantities for the 5.12-7.24 MeV nucleon ${ }^{-1}$ bin. While showing the same general trends seen in panel (a), adiabatic deceleration plays less of a role in panel (b) since the particles are scattered less and they move out of the system faster leaving adiabatic deceleration less time to work. Even so shortly after the peak of the event most of the particles in this bin started out in the 7.24-10.24 MeV nucleon ${ }^{-1}$ bin. If we ask how long after injection do particles from twice the observed energy become the largest contributor, at $0.32-0.45 \mathrm{MeV}$ nucleon $^{-1}$ this occurs at about $15 \mathrm{hr}$ (orange trace), while at 5.12-7.24 $\mathrm{MeV}$ nucleon $^{-1}$ it occurs at $\sim 40 \mathrm{hr}$. Figure 10(c) shows a continuation of this trend for the 20.48-28.96 $\mathrm{MeV}$ nucleon ${ }^{-1}$ range. In this case, at the peak of the event the particles are mostly from this same energy bin at injection, and the crossover to domination by particles of twice the $1 \mathrm{AU}$ energy (orange trace) occurs only after $\sim 90 \mathrm{hr}$. The properties shown in Figure 10 make it clear that adiabatic deceleration plays a large role in the intensity profiles at $1 \mathrm{AU}$ since at energies below a few $\mathrm{MeV}$ nucleon ${ }^{-1}$ the observed particles come from higher initial energies. Thus, initially steep spectra will lead to faster decay times, and any energy dependence in the injected particle population would reveal itself later in the event when those particles begin to appear in the lower energy bins.

Figure 11 shows effects of adiabatic deceleration on particle distributions summed over the event. In Figure 11(a), particles injected in a series of narrow energy bins (see the caption) are followed to $1 \mathrm{AU}$ and their energies are tallied. Above tens 


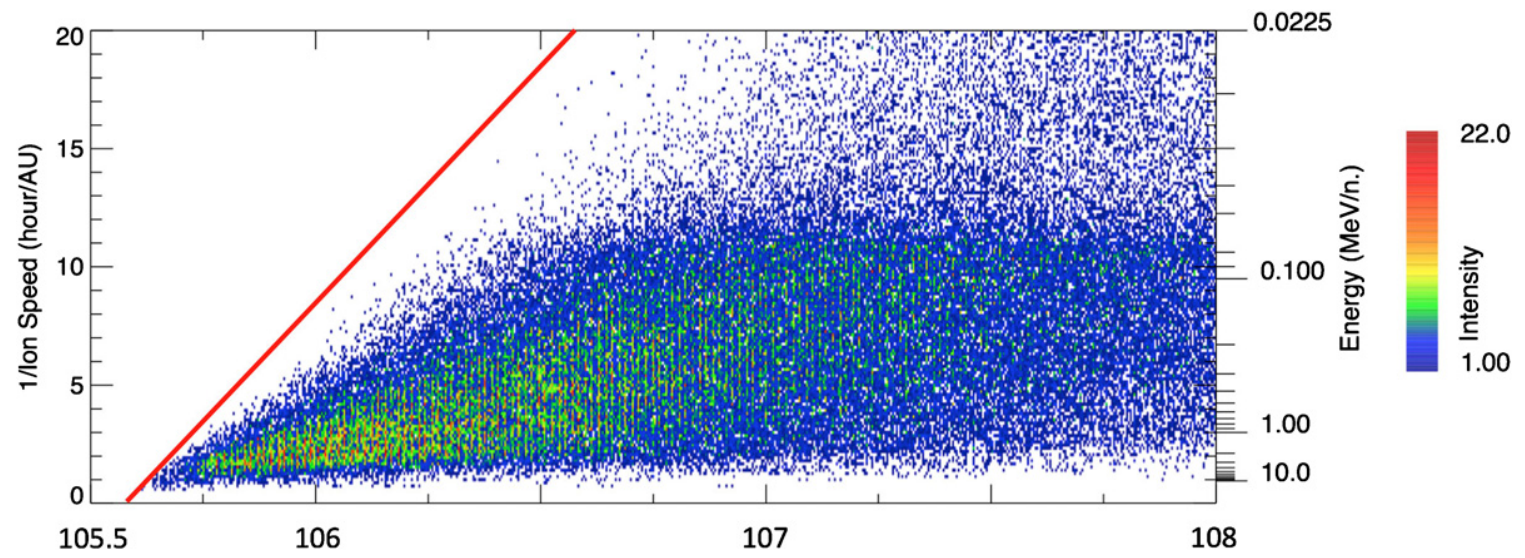

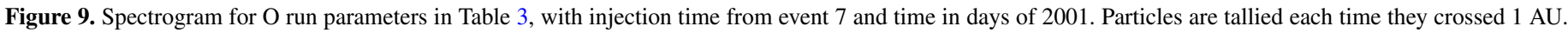
(Compare with Figure 2(b).) The red diagonal line is as in Figure 2.

of $\mathrm{MeV}$ nucleon $^{-1}$ the effects are not large, but still clearly visible, while below $1 \mathrm{MeV}$ nucleon $^{-1}$ the cooling effects are very large. Below $\sim 200 \mathrm{keV}^{\text {nucleon }}{ }^{-1}$ the particles are cooled drastically. Figure 11(b) shows the distribution of source particles that are observed at $1 \mathrm{AU}$ in the same set of narrow energy bands. The narrow observed bands actually correspond to injected particles over a much broader range at higher energies. In addition, below a few $\mathrm{MeV}$ nucleon $^{-1}$ there is a great deal of overlap in the injected distributions, which would have the effect of smearing out features in the injected spectrum.

The question arises if the significant adiabatic deceleration effects shown in Figures 10 and 11 are influenced greatly by effects near the Sun, since that is where the divergence of the solar wind is larger and the scattering is somewhat more intense due to the radial dependence of the turbulence. However, magnetic focusing effects are strongest near the Sun, quickly returning particle pitch angles to small values even after scattering. If we take particles in a given energy range at the injection and tally their energy as time evolves, there is exponential decay in energy with no large loss at early times that could signal heightened sensitivity to energy loss at small $r$. Thus, the energy loss rates derived here should be considered typical of transport in a Parker spiral geometry with typical interplanetary turbulence and solar wind speeds (see also Ruffolo 1995; Kocharov et al. 1998; Chollet et al. 2010a).

Figure 12 shows other aspects of the transport calculated over the $1 \mathrm{AU}$ particle crossings up to $200 \mathrm{hr}$ after injection, when the simulation was stopped. Figure 12(a) shows the fractional energy loss for $\mathrm{O}$ nuclei at 1 AU versus injection energy. The vertical arrow in the panel marks the injection energy (2.14 $\mathrm{MeV}^{\text {nucleon }}{ }^{-1}$ ) at which $50 \%$ of the energy is lost when observed at 1 AU. Dashed lines in panel (a) show the losses for events 5 and 10, which respectively had the most and least scattering in the survey. We note that if particle scattering keeps a particle from reaching 1 AU during the time of the simulation, its energy loss will not be tallied in Figure 12(a). Figure 12(b) shows the average number of crossings per particle and the average time between 1 AU crossings. At the lowest energies, most of the particles move past 1 AU only once and do not return; the average time between crossings has a maximum at $\sim 6 \mathrm{hr}$ near $1 \mathrm{MeV}$ nucleon $^{-1}$ and declines slowly toward higher energies, while the average number of crossings continues to grow. Figure 12(c) shows the fraction of particles versus number of crossings at $1 \mathrm{AU}$ for $\mathrm{O}$ with initial energy of 0.32-0.45 MeV nucleon ${ }^{-1}$ and 5.12-7.24 $\mathrm{MeV}_{\text {nucleon }}{ }^{-1}$ (see also Mewaldt et al. 2008; Chollet et al. 2010a). For the lower energy particles, $\sim 60 \%$ of the particles cross once only, and $80 \%$ cross three or fewer times. Particles crossing only once do not contribute to the average time between crossings shown in panel (b). The distinct even-odd variation in panel (c) shows that only a few particles $(<1 \%)$ cross 1 AU moving inward toward the Sun and fail to return due to mirroring or scattering; particles that do not return have either been absorbed at the Sun, or are still inside $1 \mathrm{AU}$ when the simulation stops. For the higher energy particles (half-filled blue squares) the number of multiple crossings is much greater, with about $25 \%$ of the particles crossing only once. The number of crossings is much larger than for the lower energy trace since the effects of convection and adiabatic cooling are less important. The chance of having an even number of crossings seems to become larger at high numbers of crossings since the even-odd difference gets smaller; however, this is likely due to the mechanics of the simulation, where particles with lots of crossings have necessarily been tallied for a long time and are more likely to be inside 1 AU (but not absorbed at the Sun) when the simulation is stopped. Even with all the multiple crossings of $1 \mathrm{AU}$, the large majority of particles are last detected moving outward from $1 \mathrm{AU}$, so there is a net outward flow that decreases with time.

Figure 13 shows calculated anisotropies for three $\mathrm{O}$ energies corresponding to the panels in Figure 10, where the anisotropy has been defined by particle pitch angle in terms of outward moving (" $F$ ") versus inward moving (" $B$ ") particle intensities: Anisotropy $=(F-B) /(F+B)$. This simple definition is used to better handle limited statistical accuracy in the simulation. The half-filled squares mark the time of maximum intensity at each energy. The plot shows that at event onset the particles are all outward moving, but by the time of the intensity maximum the anisotropy has decreased to $\sim 0.3$, with an exponential decay shown throughout most of the event. These results are typical of both simple (e.g., Schulze et al. 1977) and more complex models (Ng \& Reames 1994; Ruffolo 1995; Li et al. 2003), namely smoothly decreasing anisotropies that are initially large during the rise phase.

Propagation effects on the event-summed spectra are shown in Figure 14. Figure 14(a) shows the injected spectra for O (red) and Fe (blue) with slopes and break energy from Table 3. Figure 14(b) shows the spectra at 1 AU. Comparing panel (b) with (a) note there are fewer particles at low energies (due to cooling) and more at higher energies (due to multiple crossings). After transport high-energy slopes harden by $\sim 0.35$ units, and 

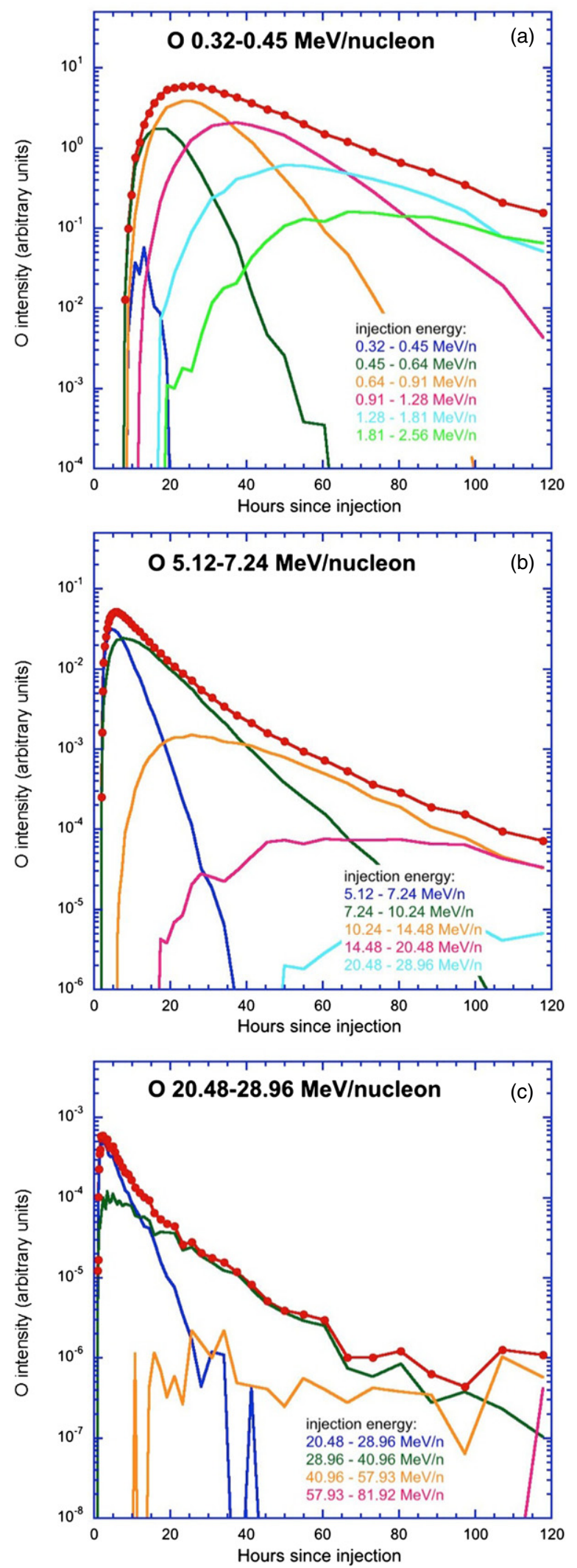

Figure 10. Filled red circles: time-intensity profiles at $1 \mathrm{AU}$ for $\mathrm{O}$ at (a) $385 \mathrm{keV}$ nucleon $^{-1}$, (b) $6.18 \mathrm{MeV}_{\text {nucleon }}{ }^{-1}$, and (c) $25.1 \mathrm{MeV}^{\text {nucleon }}{ }^{-1}$. Solid lines show the original energy profiles at injection near the Sun for particles observed at $1 \mathrm{AU}$ in the three energy bins; the filled red circle trace is the sum of the other traces. The panels show the strong effect of adiabatic deceleration, particularly at low energy. the low-energy slopes harden by $\sim 0.65$ units, typical results for fits to the events in Table 1. The break energy also decreases, from 4.0 MeV nucleon ${ }^{-1}$ at injection to $\sim 2.5 \mathrm{MeV}$ nucleon $^{-1}$ at $1 \mathrm{AU}$. We note that even though the charge-to-mass ratios of the $\mathrm{O}$ and $\mathrm{Fe}$ in the calculation differ by a factor of $\sim 2$, the eventintegrated spectra show very little difference. This implies that differences in the $\mathrm{O}$ and $\mathrm{Fe}$ spectra at $1 \mathrm{AU}$ must be primarily due to differences in the spectra at injection. Figure 14(c) shows the $\mathrm{Fe} / \mathrm{O}$ ratio at injection and $1 \mathrm{AU}$. Except for the lowering of the break energy, the ratios are virtually the same. The flat Fe/O ratios above and below the break reflect the input spectra which were identical for $\mathrm{O}$ and $\mathrm{Fe}$; if the $\mathrm{O}$ and $\mathrm{Fe}$ spectra differed leading to an energy dependent $\mathrm{Fe} / \mathrm{O}$ ratio as often observed, then the adiabatic deceleration would change the initial $\mathrm{Fe} / \mathrm{O}$ ratio since it would be smeared out towards lower energies. (We note that the intensities in panels (a) and (b) are arbitrary units and so do not show the $\sim 1 / r^{2}$ dependence of intensity between injection and $1 \mathrm{AU})$.

Figure 15 explores the extent to which the energy scaling in Equation (1), which was derived from a simple diffusion argument, is preserved in the full simulation here that is based on a much more physically complete picture of interplanetary transport and observed properties of interplanetary turbulence of the solar wind. For three representative energies, the red lines in the panel show the $\mathrm{Fe} / \mathrm{O}$ ratio computed at the same energy nucleon ${ }^{-1}$ for both species, i.e., the energy scaling parameter $=1.0$. This trace shows a significant decrease in $\mathrm{Fe} / \mathrm{O}$ during the rise phase, which is physically due to the lower charge-to-mass ratio of $\mathrm{Fe}$ versus $\mathrm{O}(0.179$ versus 0.375 from Table 3). This leads to a larger gyroradius for $\mathrm{Fe}$, and from Equation (4), a weaker pitch angle scattering and therefore a longer mean free path. Consequently, the Fe arrives early, leading to the initially high $\mathrm{Fe} / \mathrm{O}$ ratio. The other traces in Figure 15 show the $\mathrm{Fe} / \mathrm{O}$ ratio where the $\mathrm{O}$ energy has been scaled by the factor shown in the panels. The scaled energy traces at long times are all above the trace with scaling 1.0: this is due to the spectral shape since there are fewer particles at higher energies. Note that for energy scaling even as small as 1.25 that large initial spike in $\mathrm{Fe} / \mathrm{O}$ has decreased significantly, and in Figure 15(a) it has practically vanished. For the $q=5 / 3$ Kolmogorov spectral index used in the sample calculation and charge states in Table 3, Equation (1) gives energy scaling of 1.45 , which is plotted with the heavy green trace in the panels. With this scaling choice, the time dependence in the $\mathrm{Fe} / \mathrm{O}$ has essentially disappeared, but notice that the ratio for the energy scaling values of 1.75 and 1.25 is just about as flat. Figures 1 and 15 show that the scaling factor can only be roughly estimated from the adjusting the data to give a flat $\mathrm{Fe} / \mathrm{O}$ ratio during the event.

\section{FITS TO INDIVIDUAL SEP EVENTS}

The simulation model was used to fit hourly average intensity profiles for $\mathrm{H}, \mathrm{He}, \mathrm{O}$, and $\mathrm{Fe}$ for each of the 17 events from $386 \mathrm{keV}$ nucleon $^{-1}$ to $>100 \mathrm{MeV}$ nucleon $^{-1}$. In order to limit the number of free parameters, we used the same $420 \mathrm{~km} \mathrm{~s}^{-1}$ solar wind speed and Parker spiral configuration for all events; injection times were the flare maximum times in Table 1 for all species and energies. Mass-to-charge ratios used in all fits were: $\mathrm{H}=1 ; \mathrm{He}=4 / 2 ; \mathrm{O}=16 / 6$; and $\mathrm{Fe}=56 / 10$ and 56/14 (both cases calculated), based on slow solar wind values (von Steiger et al. 2000), along with the observed increase in Fe charge states typically seen in SEP events (Klecker et al. 2006). 

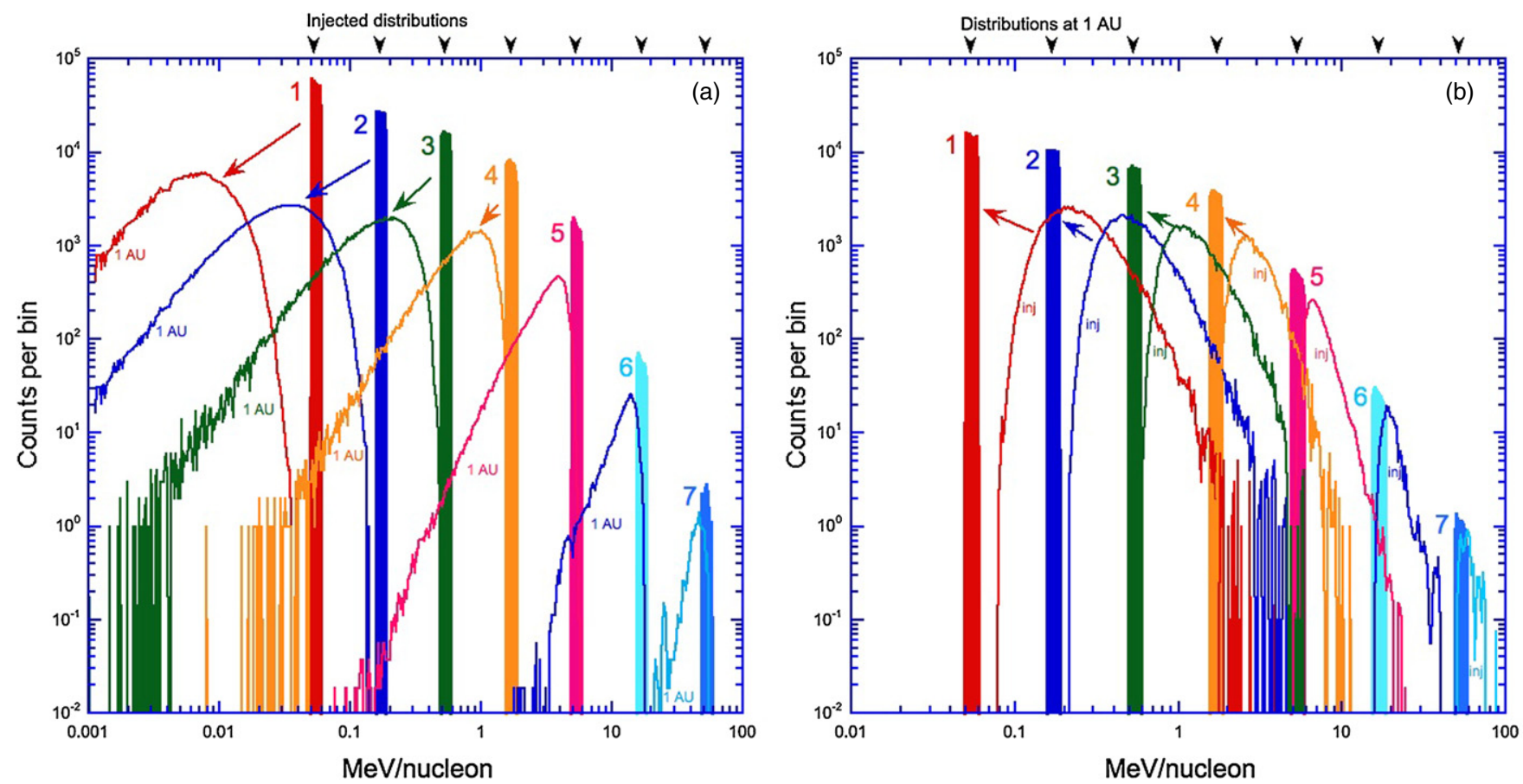

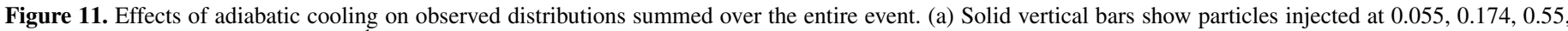

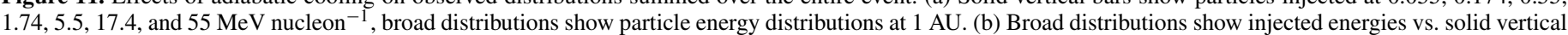
bars showing observed energy at $1 \mathrm{AU}$ in same bands as panel (a).

\subsection{Fit to 2001 April 15 (DOY 105) Event}

We use event 7 as an example of the fitting procedure. First, the spectral slopes at injection were estimated for $\mathrm{H}, \mathrm{He}, \mathrm{O}$, and Fe by fitting event-averaged spectra over linear sections at low and high energies to obtain low- and high-energy slopes at $1 \mathrm{AU}$, as well as a break energy where these two segments intersected. Then the injection spectrum slope was estimated with an index $\sim 0.5$ units steeper at low energies, and $\sim 0.3$ units steeper at high energies, and the injection break energy nucleon ${ }^{-1}$ set at $\sim 1.5$ times the $1 \mathrm{AU}$ value. This generally produced a reasonable fit to the $1 \mathrm{AU}$ observations with only small further adjustments needed. Then, the spectral index of the interplanetary turbulence spectrum, $q$, was guided by the $\mathrm{Fe} / \mathrm{O}$ energy scaling ratio (Table 2). In most cases (i.e., where the energy scaling factor was 1.4 or 2.0) we used the nominal $q=5 / 3$ Kolmogorov value since the scaling factor derived from the data is approximate and its dependence on $q$ is fairly weak for values above $\sim 1.4$ (Figure 1). Finally, the $D_{\mu \mu}$ value was estimated based on the sharpness of the rise and the decay rate. Since event 7 showed considerable scattering in its spectrogram (Figure 2(b)) the $D_{\mu \mu}$ value was chosen to give a $1 \mathrm{AU}$ scattering mean free path of $0.15 \mathrm{AU}$ for $1.08 \mathrm{MeV}$ nucleon $^{-1}$ O. $D_{\mu \mu}$ values differing by more than $10 \%-20 \%$ give significantly profiles for a given event and so can be considered a typical uncertainty for our $D_{\mu \mu}$ values (e.g., Schulze et al. 1977; Mason et al. 1983, their Figure 3; Beeck et al. 1987, their Figure 13).

Fits to event 7 spectra are shown by dashed lines in Figure 5, where each species is normalized separately. Deviations from the simulation shape arise from the fact that the actual spectra are only approximately two-segment power laws. Below $\sim 1 \mathrm{MeV}$ nucleon $^{-1}$ the $\mathrm{H}$ and $\mathrm{He}$ spectra are affected by the shock and would probably roll over more (like O) had the shock not produced particles at $1 \mathrm{AU}$. Note the Fe spectra for both $M / Q=56 / 10$ (dashed brown) and $M / Q=56 / 14$ (dashed orange) show significant differences only below $\sim 1 \mathrm{MeV}$ nucleon $^{-1}$ where transport effects are strongest; however, this does not really give evidence for charge 14 material since an adjustment of the low energy injected spectrum for charge 10 could give the same result-such fine tuning was beyond the scope of this investigation.

Figure 3 compares calculated hourly intensities for event 7 (blue lines) with the observations (filled red circles) for each species at four representative energies. Notice the shock passage (green dashed line) was associated with large intensity increases for low energy $\mathrm{H}$ and $\mathrm{He}$, followed by a steep decline in intensities. The fits were chosen to follow the rise profile and decay through the shock passage, with each fit normalized to intensities near the peak (except panels (a) and (e)). While there are deviations in most panels that could be improved individually, taken as a group we consider this fit to be reasonable given our requirement of a single turbulence spectrum to fit a broad range of energies and mass-to-charge ratios. The role of the turbulence spectral slope $q$ can be seen most easily in the column of $1.09 \mathrm{MeV}$ nucleon $^{-1}$ fits: the Fe fit has a much faster rise and a faster decay than lighter elements, since $\mathrm{Fe}$ samples different turbulence wavenumbers than lighter elements due to its mass-to-charge ratio. The bottom row shows Fe intensities for both charge 14 and 10, illustrating that this difference is most apparent at low energies. In the highest energy panels, the simulation results show fluctuations and some dropouts due to statistical limitations of the Monte Carlo calculation. Panels (a) and (e) are clearly not fitted by our simple model, which does not include effects of shockassociated increases at $1 \mathrm{AU}$, so the lowest rigidity for which there is an acceptable fit is the $1.09 \mathrm{MeV}$ protons; the highest rigidity fitted is panel (p). This corresponds to a rigidity range of 45-1152 MV.

We now turn to the temporal behavior of the $\mathrm{Fe} / \mathrm{O}$ ratio, which is shown for two energies in panel 7 of Figure 6. The 

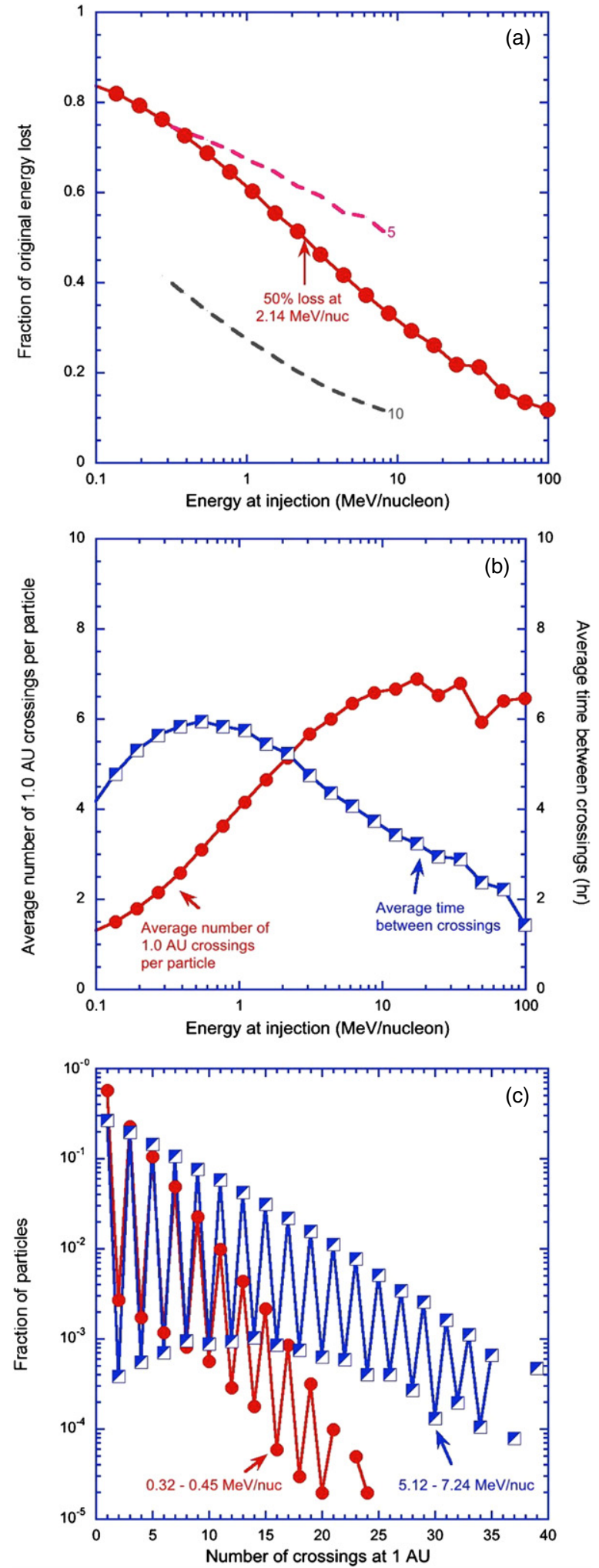

Figure 12. Simulations for $\mathrm{O}$ at $1 \mathrm{AU}$ showing (a) fractional energy loss vs. injection energy (b) average number of 1 AU crossings (filled red circles) and average time (hr) between crossings (half-filled blue squares) vs. injection energy, and (c) fraction of particles at $0.34-0.45 \mathrm{MeV}^{\text {nucleon }}{ }^{-1}$ (filled red circles) and 5.12-7.24 $\mathrm{MeV}$ nucleon $^{-1}$ (half-filled blue squares) vs. number of times particle crossed $1 \mathrm{AU}$. Dashed lines in panel (a) show losses for events 5 and 10. Lower statistical accuracy in Monte Carlo calculation results in fluctuations seen at high energies in all panels.

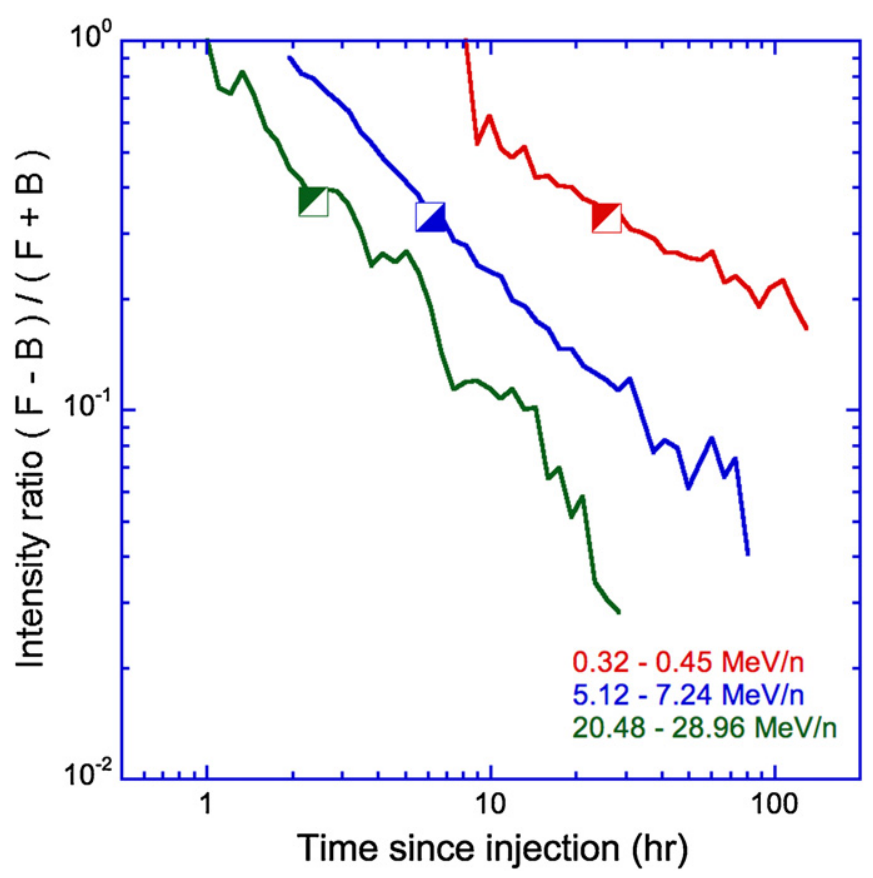

Figure 13. Calculated $\mathrm{O}$ anisotropies for red: $0.34-0.45 \mathrm{MeV}^{\text {nucleon }}{ }^{-1}$; blue: 5.12-7.24 MeV nucleon ${ }^{-1}$; and green: $20.48-28.96 \mathrm{MeV}$ nucleon $^{-1}$. Half-filled squares mark the times of maximum intensity. Note the logarithmic timescale.

filled red and blue circles are the observed $\mathrm{Fe} / \mathrm{O}$ ratios at $276 \mathrm{keV}^{\text {nucleon }}{ }^{-1}$ and $\sim 12 \mathrm{MeV}$ nucleon ${ }^{-1}$ for periods when the SEP intensities had risen well above ambient values. The green and orange lines show the simulation results summed over the same energy windows as the observations. The lowenergy green line in panel 7 generally fits the observations, which, however, show irregular fluctuations that are primarily due to fluctuations in the $\mathrm{O}$ intensity that are well outside the measurement uncertainties and presumably due to anisotropies or details of connection to the shock. The high-energy orange line in panel 7 shows the same magnitude of decrease as the data, but occurring a few hours earlier; examining Figure 3 panels (k) and (o) shows that the later decay of the observed $\mathrm{Fe} / \mathrm{O}$ is due to a slow rise of $\mathrm{O}$ in the last few hours before reaching maximum, a trend seen in some other panels in Figure 3. We consider these fits to $\mathrm{Fe} / \mathrm{O}$ reasonable, since the deviations between the simulation and data are due to irregularities in the temporal profiles that, although quite real, are presumably from processes that are not included in the model.

\section{2. $D_{\mu \mu}$ and $q$ Values for Other Events}

Table 4 lists the derived model fit parameters and Figure 16(a) shows mean free path values from Equation (5) for all the events. The mean free paths are plotted over the range of particle rigidities for which a reasonable fit was achieved (see also Columns (4) and (5) in Table 4). Note that most of the slope shown in Figure 16(a) is from the $v$ dependence in Equation (5). Figure 16(b) shows the radial dependence of the mean free path, which is significantly smaller at the injection point than at $1 \mathrm{AU}$ based on the Helios studies cited above.

Of the 17 events, 15 used the same $q=5 / 3$ value as in the 200115 April (DOY 105) event discussed above, since their energy scaling factors were all 1.4 or 2.0 (Table 2). The $D_{\mu \mu}$ values for these events ranged from 1.5 to 6.0 , thus covering a mean free path range of $0.54-0.13 \mathrm{AU}$ for $12 \mathrm{MeV}$ nucleon $^{-1} \mathrm{O}$, a difference between events that were nearly scatter-free to those 

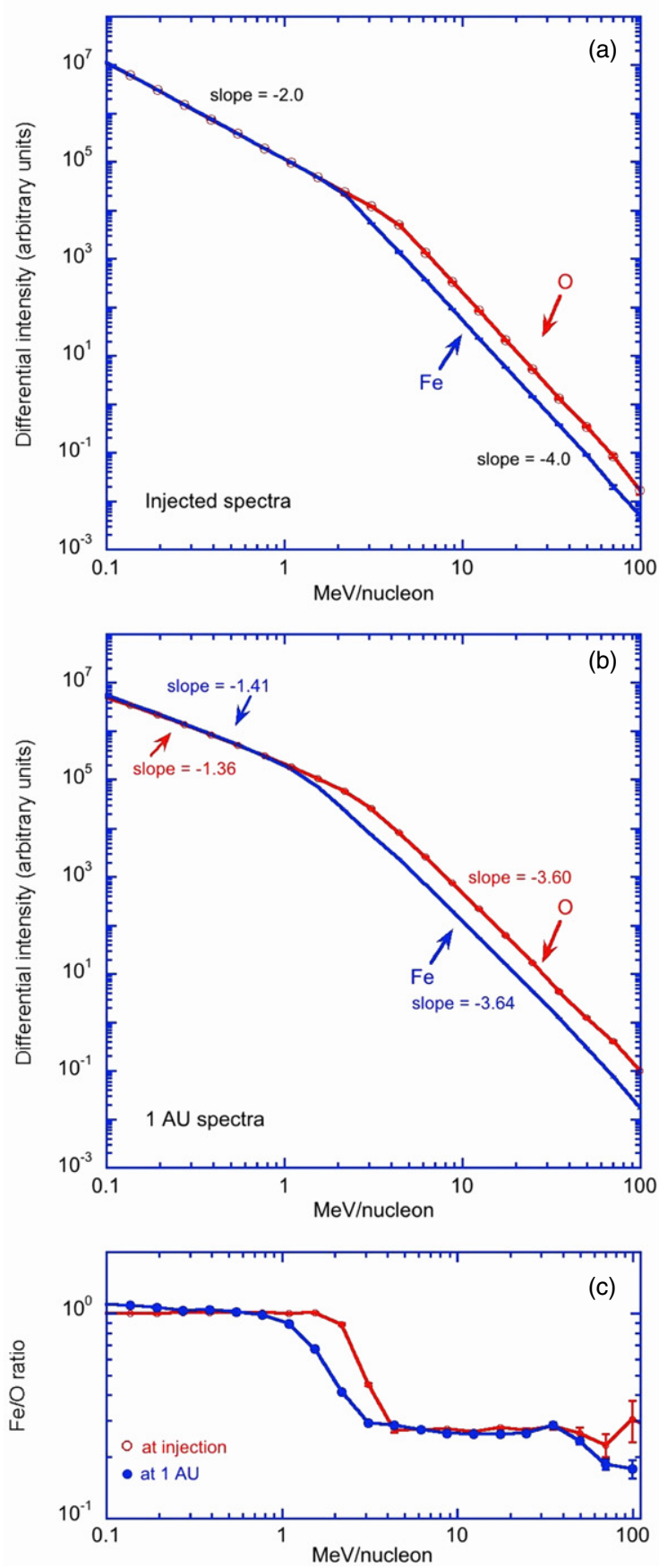

Figure 14. (a) Sample event spectral form for $\mathrm{O}$ (red) and $\mathrm{Fe}$ (blue) at injection and (b) at $1 \mathrm{AU}$ summed over entire event. The smaller number of particles in panel (b) vs. (a) is due to cooling which moves particles below the left axis of the plot. (c) $\mathrm{Fe} / \mathrm{O}$ ratio at injection (red) and $1 \mathrm{AU}$ (blue).

with lots of scattering. Considering the remaining two events, the 2000 October 25 (DOY 299) event had an energy scaling ratio of $\sim 1.0$ (i.e., no shift) and so $q=1.9$, while the 2003 November 4 (DOY 308) event had a large energy shift and so $q=1.1$. At high energies events 3 and 12 (see Table 3) had energy scaling near 1.0, and these energies were fitted with a separate $D_{\mu \mu}$ and $q$ values that joined the low-energy segments as can be seen in Figure 16. The transition rigidities between the two slopes were determined by examining fits to individual species at a range of energies. Undoubtedly such simple slope changes for the interplanetary turbulence are an oversimplification.
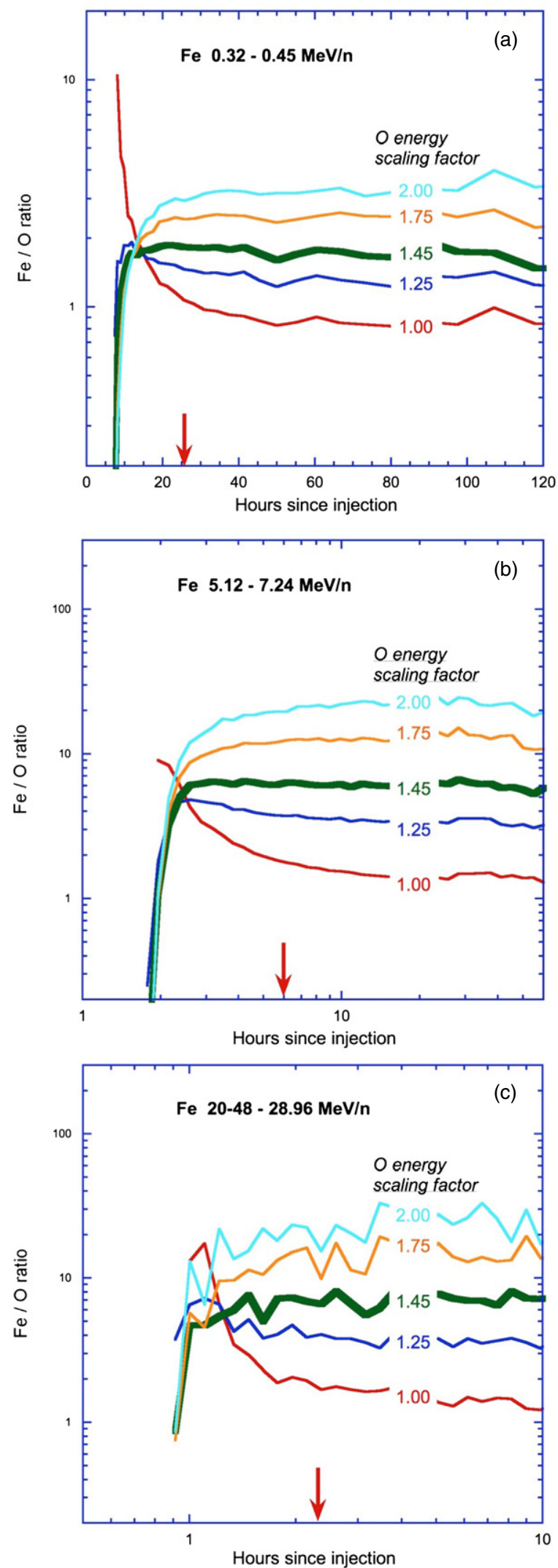

Figure 15. Average $\mathrm{Fe} / \mathrm{O}$ ratio vs. time at $1 \mathrm{AU}$ for $\mathrm{Fe}$ with energy of (a) $0.32-0.45 \mathrm{MeV}$ nucleon $^{-1}$, (b) $5.12-7.24 \mathrm{MeV}$ nucleon $^{-1}$, and (c) 20.48-28.96 MeV nucleon ${ }^{-1}$. Different color traces show scaled energy for $\mathrm{O}$ compared to Fe: e.g., trace marked 2.0 is for $\mathrm{O}$ at $2.0 \times$ the Fe energy nucleon ${ }^{-1}$ for that panel. Red arrows mark time of maximum intensity. The heavy green line shows ratio at scaled energy given by Kolmogorov index. Note the logarithmic time scale in panels (b) and (c) to show behavior at early times. 
Table 4

Model Fit Parameters

Injection Profile ${ }^{\mathrm{a}}$

Turbulence Parameters ${ }^{\mathrm{b}}$

\begin{tabular}{|c|c|c|c|c|c|c|c|c|c|c|c|c|c|c|c|}
\hline \multirow[b]{2}{*}{ Event No. } & \multirow[b]{2}{*}{ Date of Maximum } & \multirow[b]{2}{*}{ DOY } & \multirow[b]{2}{*}{$\begin{array}{l}\text { Lowest Rigidity Fitted } \\
\text { (MV) } \\
\text { (4) }\end{array}$} & \multirow[b]{2}{*}{$\begin{array}{l}\text { Highest Rigidity Fitted } \\
\text { (MV) } \\
\text { (5) }\end{array}$} & & \multicolumn{5}{|c|}{ Turbulence Parameters } & \multirow[b]{2}{*}{$\begin{array}{c}\lambda 72 \mathrm{MV}^{\mathrm{c}} \\
(\mathrm{AU})\end{array}$} & \multirow[b]{2}{*}{$\begin{array}{c}\lambda 400 \mathrm{MV}^{\mathrm{c}} \\
(\mathrm{AU})\end{array}$} & \multirow[b]{2}{*}{ Notes on Fits } \\
\hline & & & & & $\begin{array}{c}\text { Low Rigidity } \\
\text { (6) }\end{array}$ & $\begin{array}{c}\text { Transition } \\
\text { (MV) } \\
\text { (7) }\end{array}$ & $\begin{array}{c}\text { High Rigidity } \\
\text { (8) }\end{array}$ & $\begin{array}{l}D \mu \mu \\
(\mathrm{Hz}) \\
(9)\end{array}$ & $\begin{array}{c}q \\
(10)\end{array}$ & $\begin{array}{c}D \mu \mu, q \text { Transition } \\
\text { (MV) } \\
\text { (11) }\end{array}$ & $\begin{array}{l}D \mu \mu \\
(\mathrm{Hz}) \\
(12)\end{array}$ & $\begin{array}{c}q \\
(13)\end{array}$ & & & \\
\hline 1 & 1998 Мау 6 & 126 & 53 & 1152 & $\delta$ & $\ldots$ & $\ldots$ & 1.5 & 1.667 & $\ldots$ & $\ldots$ & $\ldots$ & 0.28 & 0.54 & 1,2 \\
\hline 2 & 1998 Sep 30 & 273 & 53 & 1365 & $\delta$ & $\ldots$ & $\ldots$ & 4.0 & 1.667 & $\ldots$ & $\ldots$ & $\ldots$ & 0.10 & 0.20 & 1,2 \\
\hline 3 & 1999 Jun 4 & 155 & 29 & 745 & $\delta$ & 175 & $\sigma 5.0$ & 4.0 & 1.667 & 340 & 11.0 & 1.90 & 0.10 & 0.20 & 2 \\
\hline 4 & $2000 \mathrm{Sep} 12$ & 256 & 90 & 848 & $\delta$ & 240 & step $10^{\mathrm{d}}$ & 6.0 & 1.667 & $\ldots$ & $\ldots$ & $\ldots$ & 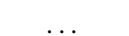 & 0.13 & \\
\hline 5 & 2000 Oct 25 & 299 & 29 & 255 & $\sigma 5.0$ & $\ldots$ & $\ldots$ & 48.0 & 1.9 & $\ldots$ & $\ldots$ & $\ldots$ & 0.03 & $\ldots$ & 1,3 \\
\hline 6 & 2001 Jan 28 & 28 & 29 & 848 & $\sigma 5.0$ & $\ldots$ & $\ldots$ & 5.0 & 1.667 & $\ldots$ & $\ldots$ & $\ldots$ & 0.08 & 0.16 & 1 \\
\hline 7 & 2001 Apr 15 & 105 & 45 & 1152 & $\delta$ & $\ldots$ & $\ldots$ & 4.0 & 1.667 & $\ldots$ & $\ldots$ & $\ldots$ & 0.10 & 0.20 & \\
\hline 8 & 2002 Apr 21 & 111 & 150 & 968 & $\delta$ & $\ldots$ & $\ldots$ & $5.5^{\mathrm{e}}$ & 1.1 & $\ldots$ & $\ldots$ & $\ldots$ & $\ldots$ & $0.17^{\mathrm{e}}$ & 4 \\
\hline 9 & $2002 \mathrm{Jul} 7^{\mathrm{f}}$ & 188 & 53 & 437 & $\delta$ & 150 & $\sigma 5.0$ & 3.0 & 1.667 & $\ldots$ & $\ldots$ & .. & 0.14 & 0.27 & 1,2 \\
\hline 10 & 2002 Aug 14 & 226 & 29 & 437 & step 15 & $\ldots$ & $\cdots$ & 0.8 & 1.667 & $\ldots$ & $\ldots$ & .. & 0.52 & 1.01 & 2 \\
\hline 11 & 2002 Aug 24 & 236 & 53 & 1365 & $\sigma 5.0$ & $\ldots$ & $\ldots$ & 4.0 & 1.667 & $\ldots$ & $\ldots$ & $\ldots$ & 0.10 & 0.20 & 1 \\
\hline 12 & 2003 Nov 4 & 308 & 53 & 823 & $\delta$ & 175 & $\sigma 5.0$ & 0.6 & 1.1 & 85 & 12.0 & 1.90 & 0.11 & 0.18 & 1 \\
\hline 13 & 2003 Dec 2 & 336 & 29 & 437 & $\delta$ & 150 & $\sigma 5.0$ & 4.0 & 1.667 & $\ldots$ & $\ldots$ & $\ldots$ & 0.10 & 0.20 & 1,2 \\
\hline 14 & 2004 Apr 11 & 102 & 110 & 848 & $\sigma 5.0$ & $\ldots$ & $\ldots$ & 1.5 & 1.667 & $\ldots$ & $\ldots$ & .. & $\ldots$ & 0.54 & 1 \\
\hline 15 & 2005 Jan 20 & 20 & 150 & 1685 & $\delta$ & $\ldots$ & $\ldots$ & 3.0 & 1.667 & $\ldots$ & $\ldots$ & $\ldots$ & . & 0.27 & 2 \\
\hline 16 & 2006 Dec 13 & 347 & 110 & 1685 & $\delta$ & $\ldots$ & $\ldots$ & 4.0 & 1.667 & $\ldots$ & $\ldots$ & $\ldots$ & $\ldots$ & 0.20 & \\
\hline 17 & 2012 Jan 23 & 23 & 110 & 848 & $\delta$ & 175 & $\sigma 6.0$ & 6.0 & 1.667 & & $\ldots$ & $\ldots$ & $\ldots$ & 0.13 & \\
\hline
\end{tabular}

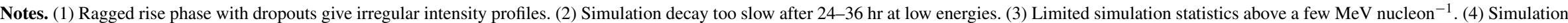
decay too slow after $24-36 \mathrm{hr}$ at high energies.

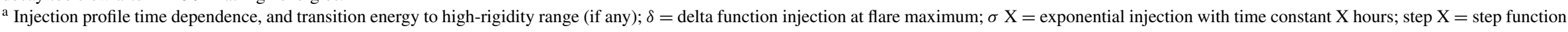
with width $\mathrm{X}$ hours.

${ }^{\mathrm{b}}$ IMF turbulence level $D \mu \mu$, power spectral slope $q$, and magnetic rigidity for transition between low- and high-energy $D \mu \mu$ and $q$ values if any.

c Scattering mean free path (AU) at $1 \mathrm{AU}$ calculated with Equation (5) for O at $386 \mathrm{keV}$ nucleon"-1 (72 MV, "low") and $12 \mathrm{MeV}$ nucleon $^{-1}$ (400 MV, "high").

${ }^{\mathrm{d}}$ Step function width broadens to $20 \mathrm{hr}$ above $340 \mathrm{MV}$; may be due to corotation rather than injection.

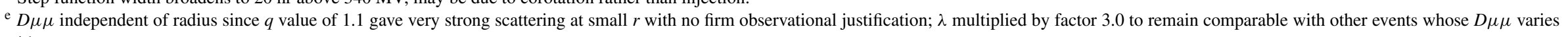
with $r$.

${ }^{\mathrm{f}}$ Fit determined primarily by protons due to low counting statistics. 

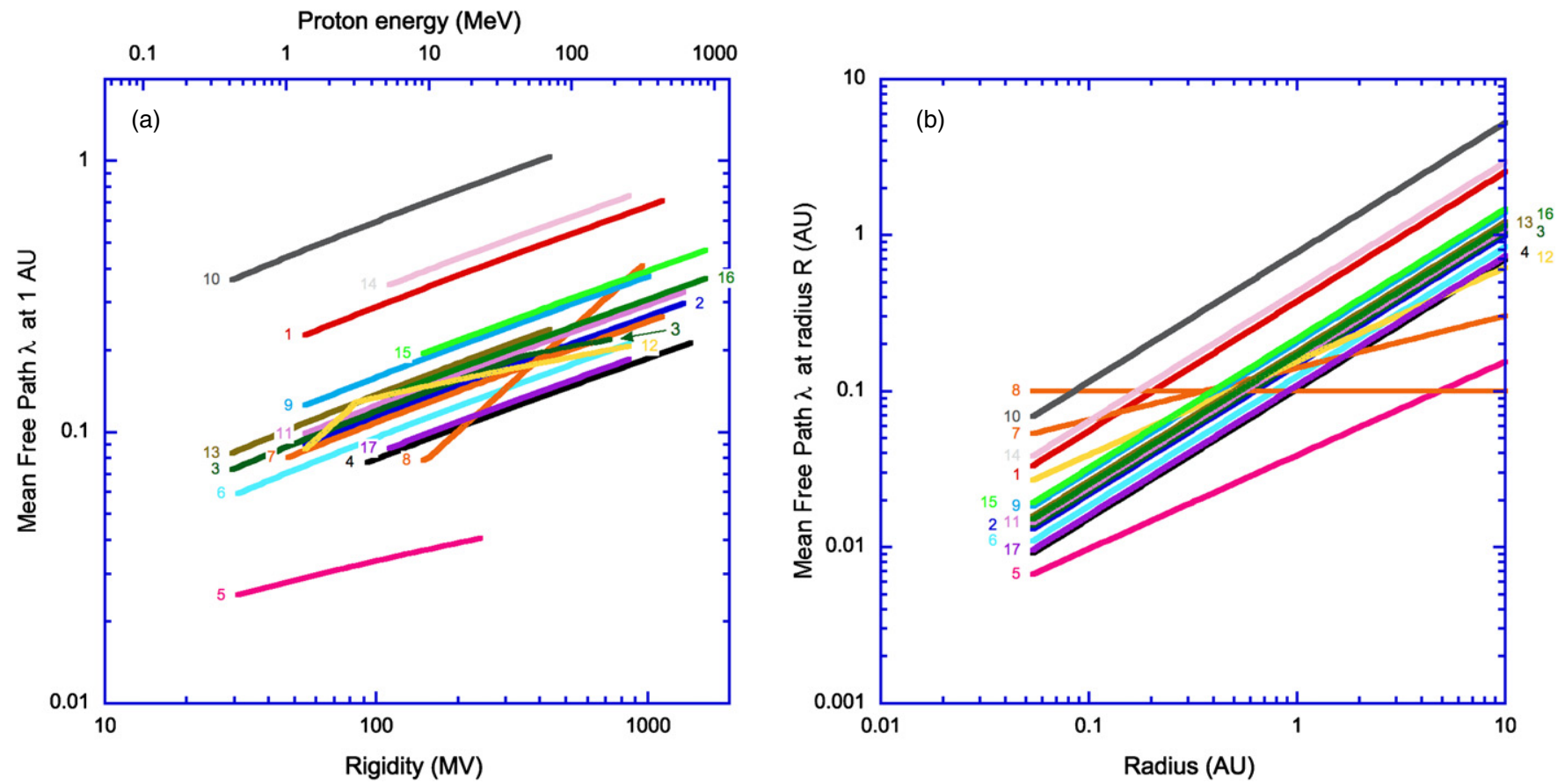

Figure 16. (a) Mean free path $\lambda$ at 1 AU for events 1-17 over rigidity range of fits (b) modeled radial dependence of $\lambda$ from injection radius to beyond 1 AU at $200 \mathrm{MV}$ (e.g., $21.2 \mathrm{MeV}$ protons, or $3.01 \mathrm{MeV}$ nucleon ${ }^{-1} \mathrm{O}^{+6}$. Events with the same $D_{\mu \mu}$ and $q$ values have been offset from each other to improve readability.
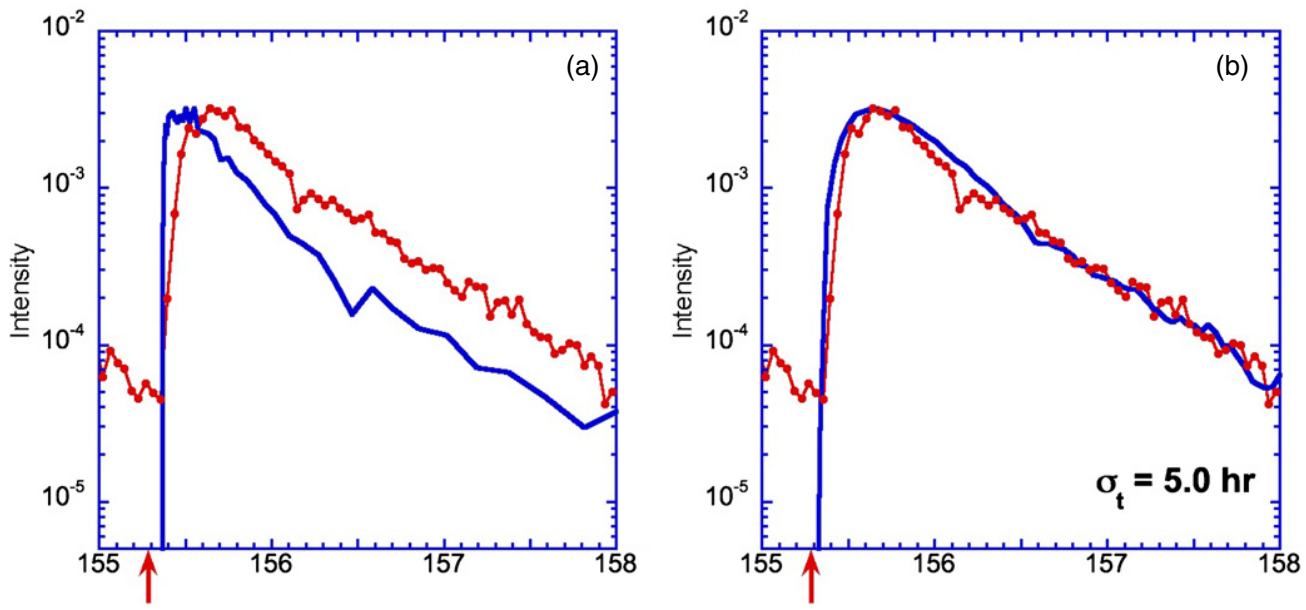

Figure 17. $8.65 \mathrm{MeV}$ nucleon ${ }^{-1} \mathrm{O}$ intensities (filled red circles) for the 1999 June 4 event (event 3, DOY 155) along with simulation results (blue line) for (a) $\delta$-function injection and (b) exponential injection with time constant $5.0 \mathrm{hr}$. The red arrow marks time of injection at flare maximum.

\subsection{Extended Injections}

Of the 17 events; 7 were satisfactorily fitted with $\delta$-function injections over their entire energy range, and 12 of the 17 events were satisfactorily fitted with $\delta$-function injections at low energies (see Table 4, Columns 6-8). But for other events systematic deviations from the simple model were apparent. Figure 17 shows an example where the left panel shows $8.65 \mathrm{MeV}$ nucleon $^{-1} \mathrm{O}$ observations (red filled circles) and the model fit with $D_{\mu \mu}$ and $q$ values that gave satisfactory fits to the low-energy intensities and $\mathrm{Fe} / \mathrm{O}$ ratio. However, at $8.65 \mathrm{MeV}$ nucleon $^{-1}$ the simulation gives a rise that is too fast and a decay whose time constant is reasonable, but which occurs many hours too soon. It is well known that the simulation profile in such cases can be suitably modified with an extended injection with a fast rise followed by an exponential decay (Reid 1964; Axford 1965; Beeck et al. 1987) or a step function (Feit
1973; Schulze et al. 1977). Extended injection intensity profiles were obtained by taking the $\delta$-function simulation and convoluting each particle with either an exponential form or a step function form of adjustable width. Figure 17(b) shows the convoluted fit with a fast rise followed by an exponential injection with decay constant $\sigma_{t}=5.0 \mathrm{hr}$; note that the fit to both the rise and decay has improved markedly. Table 4 , Columns $6-8$ show the step function or exponential widths used in the fits. We note that the physical origin of extended injections could be due to extended shock acceleration as discussed below in Section 5.1.

\subsection{Event 8 Radial Dependence of Turbulence}

Event 8 stands out in Figure 16(b) since the mean free path is independent of radius. This arises from a limitation of the simulation wherein for this event the low value of $q$ at high energies, along with a small mean free path at $1 \mathrm{AU}$ 
(Table 4) extrapolated to an extremely small mean free path near the injection point. This caused numerical problems in the simulation. Since the assumed radial dependence of the interplanetary turbulence that causes the smaller mean free path at small $r$ is an extrapolation from limited observations, we decided to set $D_{\mu \mu}$ independent of radius for this case. To keep the $1 \mathrm{AU}$ mean free path in Figure 16(a) comparable to the other events in the survey, we multiplied the mean free path value obtained with Equation (5) by 3.0, which roughly offsets the decrease in mean free path at small radius.

\subsection{Accuracy of Event Fits}

The fit parameters in Table 4 were obtained subjectively by comparing the spectra and hourly intensities with the observations for the four species and energy ranges as shown in Figure 3, and sometimes going to higher energy if statistics permitted it (see Table 4, Column 5). The spectra for each species were fit reasonably well in all cases. The hourly intensities compared with the simulation results often agreed well, but in other cases systematically deviated as follows. First, in events where there was apparently trapping of low-energy particles near the shock, no comparison was possible and this set a lowest rigidity fitted that is listed in Column 4. Other types of deviations are noted in Column 16. In several cases, the spectrogram rise phases (e.g., Figure 4(d)) showed ragged edges presumably from anisotropies and/or changing connection to the acceleration site. Such details are not included in our simple model and led to deviations between fits and observations during the rise. In cases where these deviations were severe, no fit was feasible and the Column 4 entry was adjusted accordingly. The main other deviation type was during the decay phases where after a day or so the simulation decay was too slow; eight events in the table have this type of deviation, and since their average origin is at $\mathrm{W} 71^{\circ}$ it is likely that this is a corotation effect as the event moves further from the observer and after a day or two has moved $\sim 20^{\circ}$ or more from connection to Earth, making the effects easily visible (see, e.g., discussions in Lario et al. 1998; Dröge et al. 2010).

\subsection{Summary of Fe/O Ratio Temporal Variations}

We now consider the $\mathrm{Fe} / \mathrm{O}$ temporal variations from the fits obtained with parameters summarized in Table 4. In our original survey (Mason et al. 2006) event 11 at low energy and events 5, 9, 10, and 13 at high energy were already noted as having irregular $\mathrm{Fe} / \mathrm{O}$ ratio profiles, or insufficient statistical accuracy to determine the temporal behavior, and those features can be seen in Figure 6. At low energies, event 4's Fe/O ratio decreases overall by a factor of three, but the simulation predicts a larger drop. Overall we consider 11 of the 13 low-energy fits to be reasonable, and all 13 showed decreases in $\mathrm{Fe} / \mathrm{O}$ (events 14-17 not fitted at low energies due to influence of the shock). Turning to the high-energy observations (blue filled circles) and fits (orange lines), except for the events previously cited, the other cases give reasonable fits to the magnitude of the $\mathrm{Fe} / \mathrm{O}$ ratio decrease, although often the simulation $\mathrm{Fe} / \mathrm{O}$ decrease occurs earlier than the observed change (e.g., 2, 7, 11, 14, and 16). These differences appear to arise from details of the $\mathrm{O}$ and Fe intensity profiles not captured in the model (e.g., irregularities in the rise phase; see discussion of event 7 in Section 4.1).

\subsection{He/H Temporal Variations}

Figure 7 showed the low- and high-energy hourly average $\mathrm{He} / \mathrm{H}$ ratios. It is known that the $\mathrm{He} / \mathrm{H}$ ratio sometimes exhibits a decrease during the rise phase of an event, but also it sometimes shows increases (Witte et al. 1979; Mason et al. 1983). This behavior has been modeled as due to proton-amplified waves which perturb the interplanetary turbulence spectrum resulting in the decrease in He/H (e.g., Ng et al. 1999; Tylka et al. 1999; Reames et al. 2000; Lee 2005). Since proton-amplified waves depend on the proton event size, we have ordered the panels in Figure 7 in terms of increasing peak proton flux greater than $10 \mathrm{MeV}$ value (Table 1) from smallest (event 5) to largest (event 17). Red asterisks mark events with peak proton intensity exceeding the event modeled for proton-amplified waves by Tylka et al. (1999). The simulation curves shown are similar to the cases for $\mathrm{Fe} / \mathrm{O}$, as expected since the difference in mass-tocharge ratio for $\mathrm{He}$ versus $\mathrm{H}$ is similar to that for $\mathrm{Fe}$ versus $\mathrm{O}$, and in the simulation only the mass-to-charge ratio distinguishes particles of the same speed. Comparing Figures 6 and 7, it is clear that the $\mathrm{He} / \mathrm{H}$ ratio is much less ordered than $\mathrm{Fe} / \mathrm{O}$, with several events showing increasing $\mathrm{He} / \mathrm{H}$ at low energies. In general, the events with smaller peak proton intensity show more ordered behavior than the larger events, with some notable exceptions such as event 2 .

\section{DISCUSSION}

\subsection{Injection and Interplanetary Transport}

Our interplanetary transport model produced intensity profiles that compared reasonably with observations, using simple assumptions such as $\delta$-function injection at the flare X-ray maximum and only varying particle injection spectra and interplanetary turbulence parameters for each case. By combining data from four instruments the observations covered a much broader range of energies and species than any prior study of which we are aware. When we integrate the turbulence parameters derived for the fits here to derive interplanetary scattering mean free paths, they are similar to prior work on other SEP events (e.g., discussion in Palmer 1982 and references therein; Mason et al. 1983; Beeck et al. 1987; Wanner \& Wibberenz 1991a; Bieber et al. 1994).

Adiabatic deceleration plays a significant role especially at energies below a few $\mathrm{MeV}$ nucleon $^{-1}$. For an observer at $1 \mathrm{AU}$ measuring particles in a fixed energy window, the particles observed as the event continues are coming from higher and higher initial energies as the event progresses. This could produce complex time dependences if, e.g., abundance ratios varied with energy at injection. For a case with moderate scattering $(\mathrm{mfp} \sim 0.1 \mathrm{AU})$ below $\sim 0.5 \mathrm{MeV}$ nucleon ${ }^{-1}$ particles are already significantly cooled by the time they reach $1 \mathrm{AU}$ and so there is little or no information about such low-energy ions at injection since their intensities are buried by higher energy cooled particles in a manner analogous to low-energy galactic cosmic ray modulation in the solar wind (e.g., Goldstein et al. 1970; Li et al. 2009a). The cooling also smears out the particle distributions broadly, and so would largely remove any energy dependence in the source population, e.g., of the particle ionization states below $\sim 200 \mathrm{keV}$ nucleon $^{-1}$. However, we emphasize this consideration applies to particles injected near the Sun followed by significant interplanetary scattering, and would therefore not apply in SEP events where scattering is small (e.g., Kartavykh et al. 2007) or where particles are accelerated locally as in shock passages or corotating interaction regions (e.g., Hovestadt et al. 1982; Möbius et al. 2002)

Even though adiabatic deceleration has significant effects on the low-energy particles, it was not sufficient to cause the 
spectral breaks seen at $1 \mathrm{AU}$ at a few $\mathrm{MeV}$ nucleon $^{-1}$. A significant conclusion from our simulation study is that although the spectra are modified by interplanetary transport, spectral breaks are nevertheless present at injection, suggesting they are caused by the underlying acceleration mechanism. Extended injections were found to be necessary in some events to obtain better fits to the rise or decay phase. These have been used in prior studies to better fit the rise or width of the intensity profiles (Beeck et al. 1987) or to fit observed long lasting anisotropies (Schulze et al. 1977); the time constants they used were typically $3-10 \mathrm{hr}$, similar to the injection time constants used here. The physical mechanism for these extended injections has been ascribed to acceleration timescales associated with CME heights above the corona (Kahler 1994), and these have been incorporated into models of SEP acceleration (e.g., Li et al. 2003; Lee 2005, and references therein). We point out that the timescale of the extended injections is nevertheless short enough to be consistent with most of the SEP acceleration taking place relatively close to the Sun. This may also be why our results are qualitatively similar to studies involving moving shock sources (e.g., Ng et al. 1999; Li et al. 2003, 2005)

Deviations between the observations and model simulations were most common at low energies where effects of shock trapping, anisotropies, connection to the acceleration site, and corotation (due to long event duration) are most pronounced. With such limitations in mind we conclude that the propagation model itself gives a reasonable description of the interplanetary transport of SEPs and that the transport parameters derived are representative of large western hemisphere SEP events.

\subsection{Enhanced Fe/O Ratio During SEP Rise Phases}

The physical mechanisms of interplanetary transport incorporated in our simulation model confirm that the larger gyroradius of Fe compared to $\mathrm{O}$ will lead to early arrival of Fe in SEP events provided that there is significant scattering in the interplanetary medium. We find that the temporal behavior of $\mathrm{Fe} / \mathrm{O}$ is not only qualitatively consistent with the model, but also in many cases the magnitude and/or duration of the enhancement are fitted by the model.

However, by itself this does not rule out an alternate mechanism where Fe-rich material accelerated in one SEP event is followed by material accelerated by interplanetary shocks, as suggested by Cane et al. (2003) and others (e.g., Cliver et al. 2004; Li \& Zank 2005, and references therein). In this scenario the initial "flare" material is enriched in Fe, leading to high $\mathrm{Fe} / \mathrm{O}$ early in the event, which then decreases as the interplanetary shock-accelerated material dominates the intensity. It is clearly possible for such a sequence of events to take place, and in recent years it has become clear that low intensity ${ }^{3} \mathrm{He}$-rich and Fe-rich SEP material is being released from the Sun a large fraction of the time during active periods (Wiedenbeck et al. 2003, 2005; Kocharov et al. 2008), and even on occasion when solar activity is very low (Mason et al. 2009). Indeed, Table 2 notes that in six of the events in the current study, ${ }^{3} \mathrm{He}$-rich and $\mathrm{Fe}$-rich ambient material was clearly present in the period immediately preceding the large SEP event. So when a western hemisphere CME-associated SEP occurs, the ${ }^{3} \mathrm{He}$ - and Fe-rich suprathermals could be accelerated as well and this is known to occur on occasion (Cohen et al. 1999; Mason et al. 1999). However, in large SEP events where ${ }^{3} \mathrm{He}$ is accelerated it appears in small amounts and so appears only as a tracer, whereas to cause a large $\mathrm{Fe} / \mathrm{O}$ ratio the preceding population needs to be comparable to the material accelerated in the CME-associated event (see also Mewaldt et al. 2006).

While the two-component scenario does not make specific predictions, it should be the case that the $\mathrm{Fe} / \mathrm{O}$ ratio would be expected to be similar to that found in ${ }^{3} \mathrm{He}$-rich SEP events, i.e., $\sim 1$. Examining Figure 6 , this is sometimes the case, but at low energies in many cases $\mathrm{Fe} / \mathrm{O} \gg 1$, reaching values as high as $\sim 10$ at low energies. While such ratios are not unheard of in ${ }^{3} \mathrm{He}$-rich SEP events, it is unusual, yet in our survey about a third of the events show such high values at low energies. Comparing the Figure 6 events with initial $\mathrm{Fe} / \mathrm{O} \gg 1$, there is almost a one-to-one correspondence with events that had clearly preceding ${ }^{3} \mathrm{He}$-rich and Fe-rich material, which are marked with red asterisks (see also Table 2). However, such an association does not distinguish between simple addition of two populations, vs. acceleration of an enriched suprathermal population in the large event, which could also be the case and is consistent with the generally high $\mathrm{Fe} / \mathrm{O}$ ratios late in the events with preceding ${ }^{3} \mathrm{He}$-rich and $\mathrm{Fe}$-rich material.

Are there other compositional signatures besides high $\mathrm{Fe} / \mathrm{O}$ that could be used to test if the large SEP events have impulsive material during the rise phase? The $\mathrm{O} / \mathrm{He}$ ratio can be used since these two species also have different mass-to-charge ratios and a decrease similar to $\mathrm{Fe} / \mathrm{O}$ (but smaller) is expected from our propagation model; in contrast, the $\mathrm{O} / \mathrm{He}$ ratio in ${ }^{3} \mathrm{He}$ rich events is somewhat depleted compared to large SEP events (Mason et al. 1986; Reames 1999) and so in the two component scenario at most a small increase in $\mathrm{O} / \mathrm{He}$ is expected. Figure 18 shows $\mathrm{O} / \mathrm{He}$ ratios in a manner similar to Figure 6, along with predictions of the simulation. In almost all of the cases, $\mathrm{O} / \mathrm{He}$ decreases, and the amount of the decrease is in many cases fitted with the simulation model calculations. An interesting exception, event 5, may show an increase in $\mathrm{O} / \mathrm{He}$, and it also is unusual in that the $\mathrm{Fe} / \mathrm{O}$ changes are small. Taking all the evidence together, we conclude that the $\mathrm{Fe} / \mathrm{O}$ ratio and $\mathrm{O} / \mathrm{He}$ decreases are better explained as a transport effect. However, $\mathrm{He} / \mathrm{H}$ does not fit as well into this pattern for many events, and since $\mathrm{He}$ appears to behave similarly to $\mathrm{O}$ and $\mathrm{Fe}$, this identifies $\mathrm{H}$ as the most likely cause for the less systematic behavior of the $\mathrm{He} / \mathrm{H}$ ratio. $\mathrm{Ng}$ et al. (1999) and Lee (2005) have modeled this behavior of $\mathrm{H}$ as being caused by wave excitation by the streaming SEP protons.

\subsection{Broader Application of Energy Scaling}

Figure 6 showed that the simulation model sometimes fits the size of the $\mathrm{Fe} / \mathrm{O}$ decrease but not the timing, and the question arises in such cases as to what extent the model fit can be considered successful. This is explored further in Figure 19 where the $\mathrm{Fe} / \mathrm{O}$ ratios from Figure 6 have been plotted where the $\mathrm{O}$ energy nucleon ${ }^{-1}$ is about twice the $\mathrm{Fe}$ energy nucleon ${ }^{-1}$. It can be seen that in almost all cases there is a significant reduction in the $\mathrm{Fe} / \mathrm{O}$ temporal changes as was reported in the prior survey (Mason et al. 2006). Considering especially the high energies, in Figure 6 events 2, 7, 11, 14, and 16 have observed $\mathrm{Fe} / \mathrm{O}$ decreases that occur later than the simulation result; the decrease in $\mathrm{Fe} / \mathrm{O}$ is greatly reduced for these events in Figure 19. In some cases (events 1, 15, 16), there is an initial rise qualitatively similar to those shown in Figure 15 (see also Tylka et al. 1999). This seems to indicate that energy scaling feature persists even in the presence of other mechanisms that affect the fit timing features (ragged rise phase, corotation). 

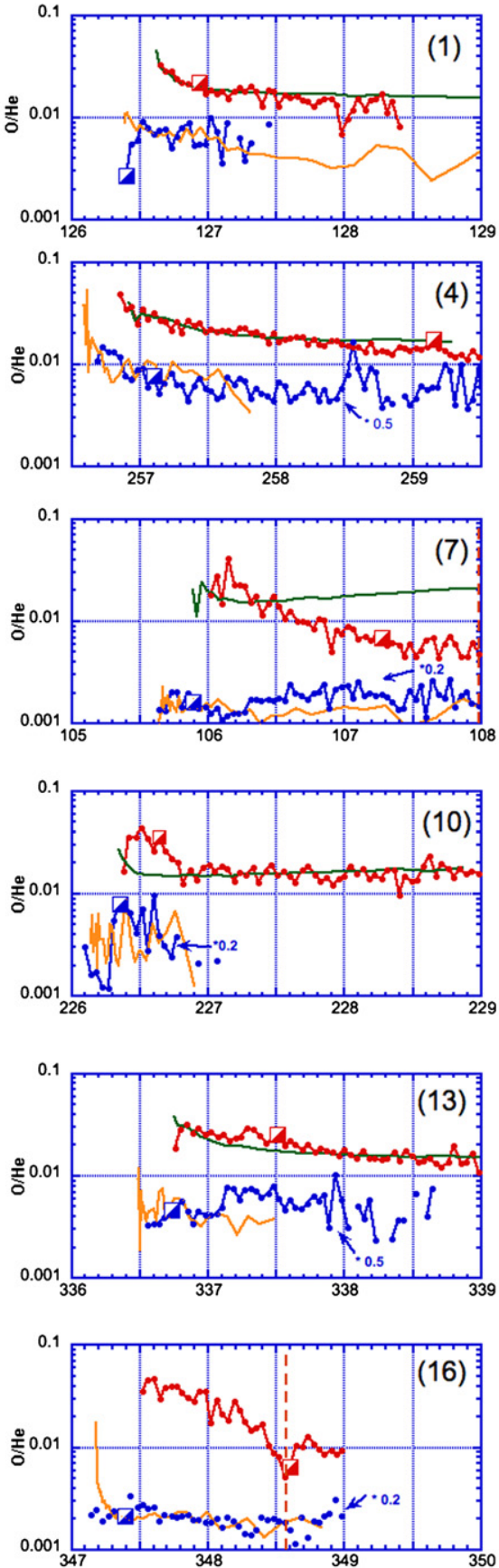
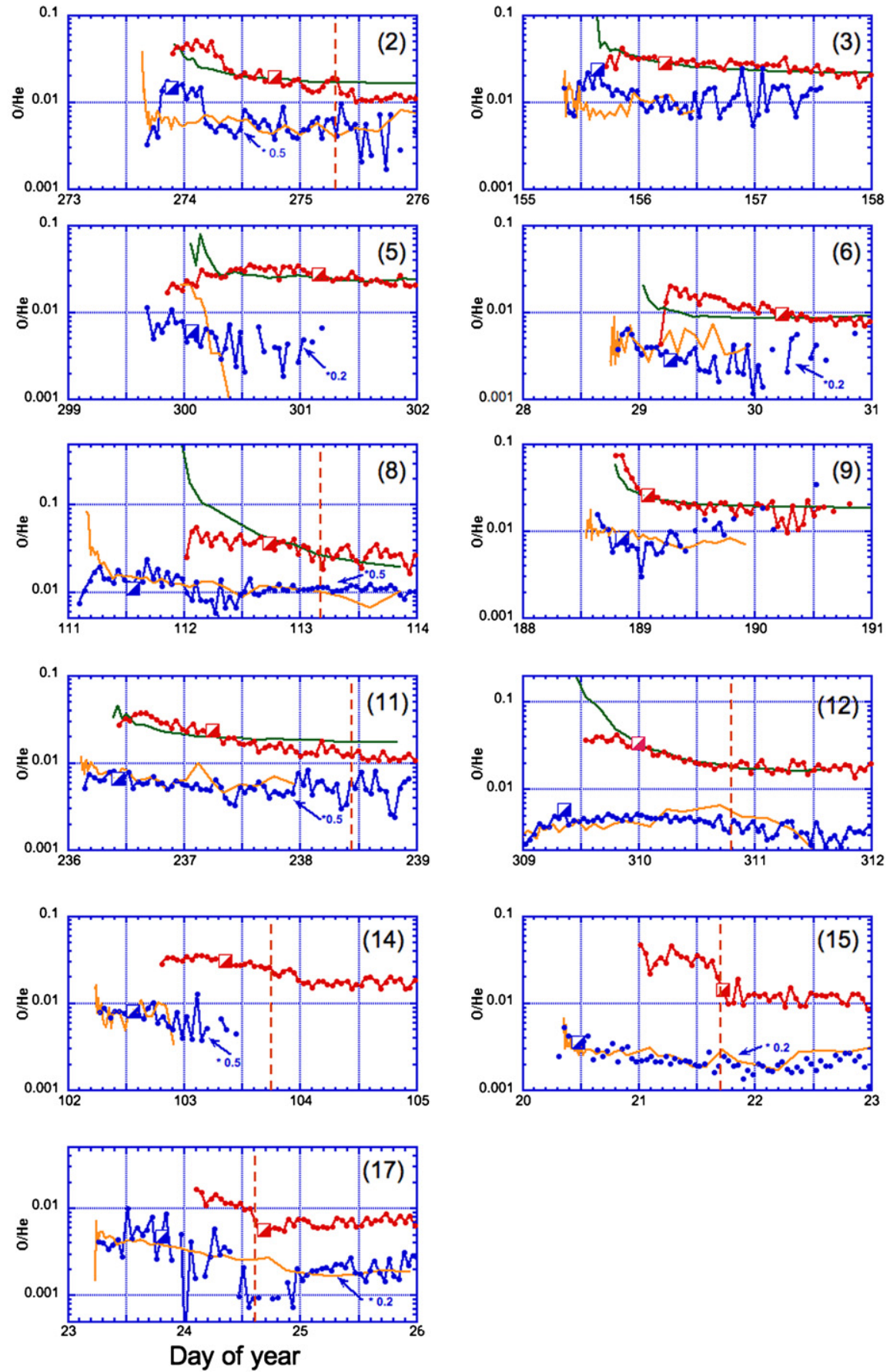

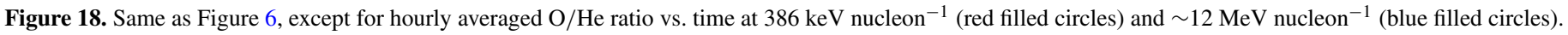

Figure 20 explores another aspect of energy scaling that goes beyond comparing classical SEP intensity profiles rise phases. Panel (b) shows low-energy $\mathrm{Fe}$ and $\mathrm{O}$ at the same energy nucleon ${ }^{-1}$ for event 12 ; not only is the $\mathrm{Fe}$ (blue) much higher than $\mathrm{O}$ for many hours before the $\mathrm{O}$ rises, but also the shapes of the $\mathrm{Fe}$ and $\mathrm{O}$ intensity profiles are quite different. This event had a large energy scaling value of 2.8 (Mason et al. 2006) which is applied to the $\mathrm{O}$ data in panel (c). Note that in panel (c) the shape of the $\mathrm{O}$ intensity has changed considerably. This leads to the flat $\mathrm{O} / \mathrm{Fe}$ ratio shown below panel (c). The scaling works also if taken in the opposite sense as panel (a) where $\mathrm{Fe}$ has been scaled to a lower energy to compare with the $\mathrm{O}$; again the shape of the $\mathrm{Fe}$ has changed leading to the much flatter ratio shown below. We see from this that the $\mathrm{Fe}$ and $\mathrm{O}$ data intensity profiles are more nearly similar when energy scaling is used rather than the usual case of the same energy nucleon ${ }^{-1}$.

Figure 21 examines energy scaling at high energies over a strong shock event used by Cane et al. (2003, p. 2) as an example of an event that consisted of an "Fe-rich component at the time of the flare and later, a shock-associated component with a lower $\mathrm{Fe} / \mathrm{O}$ ratio." This describes the feature in Figure 21(b) where $\mathrm{Fe}$ is enriched early the event, then much less so later on during the shock passage. Figures 20(a) and (c) show this same event where in panel (a) the Fe energy is scaled, and in panel (c) the $\mathrm{O}$ energy is scaled by roughly a factor of two (the scaling factors were chosen from the existing $A C E /$ SIS online data and were 

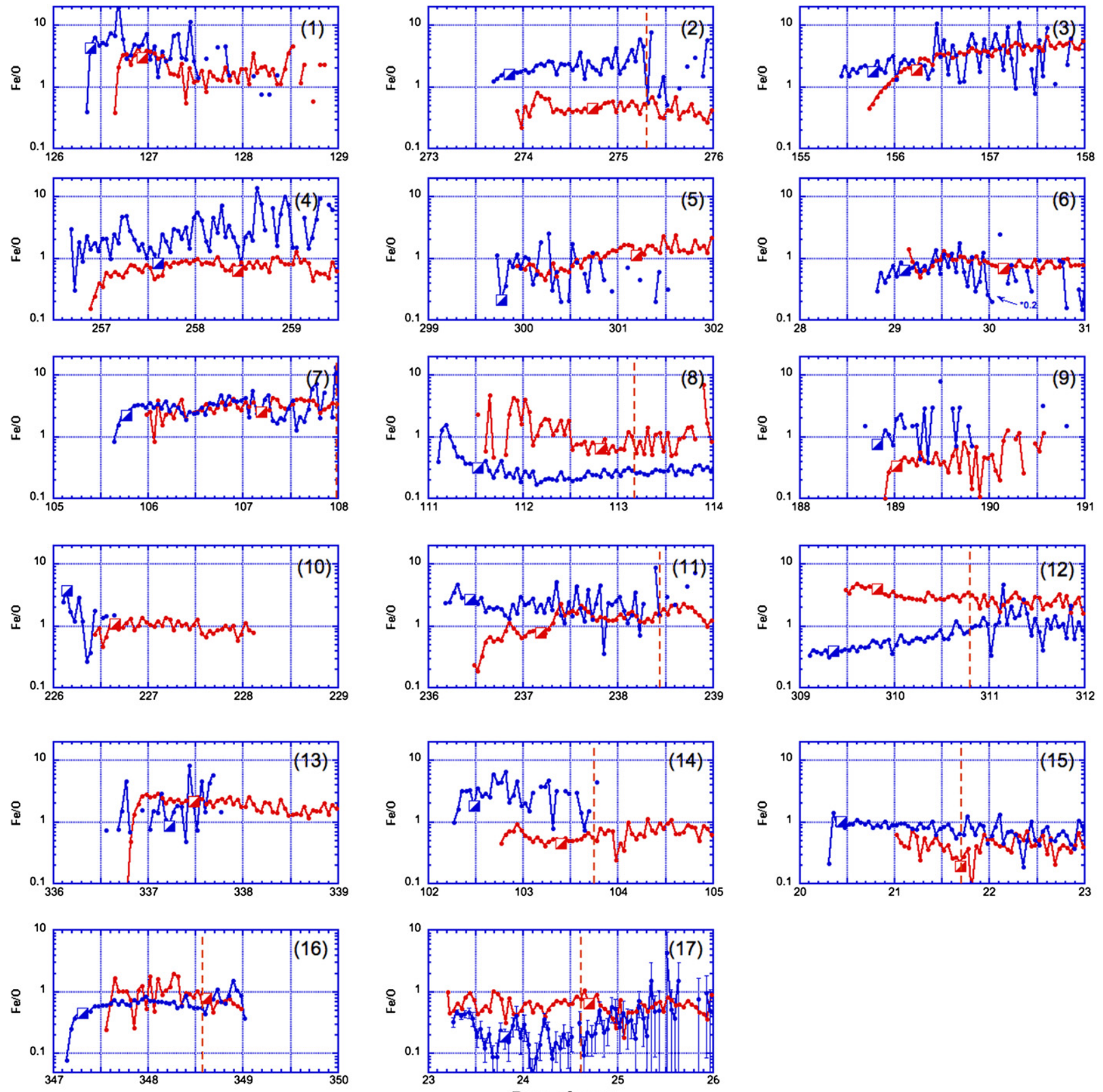

Day of year

Figure 19. Hourly average $\mathrm{Fe} / \mathrm{O}$ ratios using scaled energies: red filled circles-276 keV nucleon ${ }^{-1} \mathrm{Fe}$ divided by $542 \mathrm{keV}^{\mathrm{nucleon}}{ }^{-1} \mathrm{O}$; blue filled circles-13 MeV nucleon ${ }^{-1} \mathrm{Fe}$ divided by $24.8 \mathrm{MeV}$ nucleon ${ }^{-1} \mathrm{O}$. Dashed vertical red lines mark the time of the shock passage at $A C E$.

not fine tuned). The scaled energy results present a significantly different picture, where Fe and $\mathrm{O}$ track each other rather closely throughout the period. Thus, the inference that this event is originally enriched in Fe depends critically on the assumption that comparing the two species at the same energy nucleon ${ }^{-1}$ is appropriate. Given the close similarity between the Fe and O temporal profiles in panels (a) and (c) it appears that scaled energy is a more appropriate variable by which to display the data. Since it is clear that the propagation model used here is not appropriate for the shock passage in this event, the fact that $\mathrm{Fe} / \mathrm{O}$ varies much less over the course of the event after energy scaling is applied is an additional feature outside the scope of our propagation model.

\subsection{Implications of Energy Scaling for SEP Abundance Determinations}

Figure 14 showed that for an event where the original spectral segments had identical slopes and there was significant interplanetary scattering, the $1 \mathrm{AU}$ event-averaged spectral forms of species with different charge-to-mass ratios differed only by small amounts. Thus, a ratio such as $\mathrm{Fe} / \mathrm{O}$ was little changed except for the location of the break energy, which moved downward due to adiabatic deceleration. There is, however, an effect present since the $\mathrm{O}$ is more strongly cooled than the $\mathrm{Fe}$, so if we consider identical energy windows for $\mathrm{O}$ and $\mathrm{Fe}$ at $1 \mathrm{AU}$, the initial particle energies will be different, 

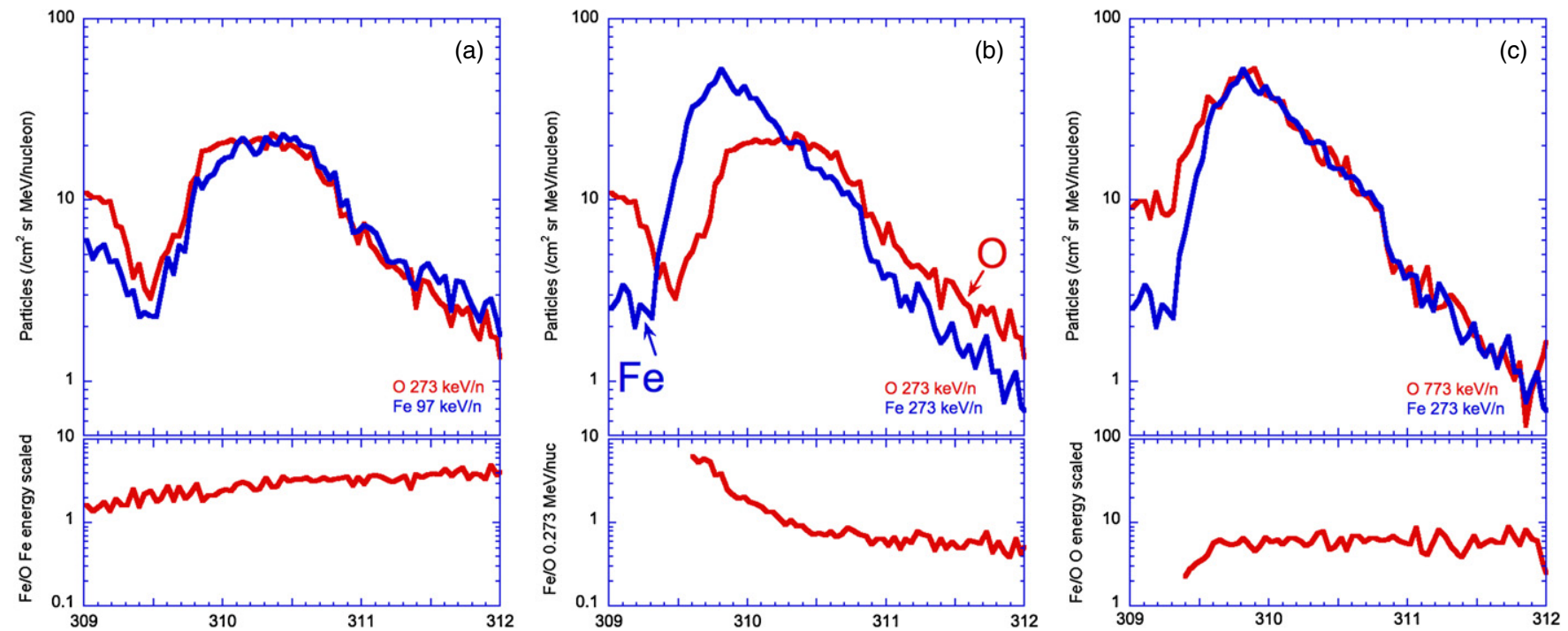

Figure 20. Upper panels: hourly average $\mathrm{Fe}$ (blue) and $\mathrm{O}$ (red) for event 12: (a) $\mathrm{O}$ at reference energy, Fe energy scaled; (b) $\mathrm{Fe}$ and $\mathrm{O}$ at $273 \mathrm{keV}$ nucleon ${ }^{-1}$ reference energy; (c) Fe at reference energy, $\mathrm{O}$ energy scaled. Bottom panels: $\mathrm{Fe} / \mathrm{O}$ ratios for intensities in upper panels.
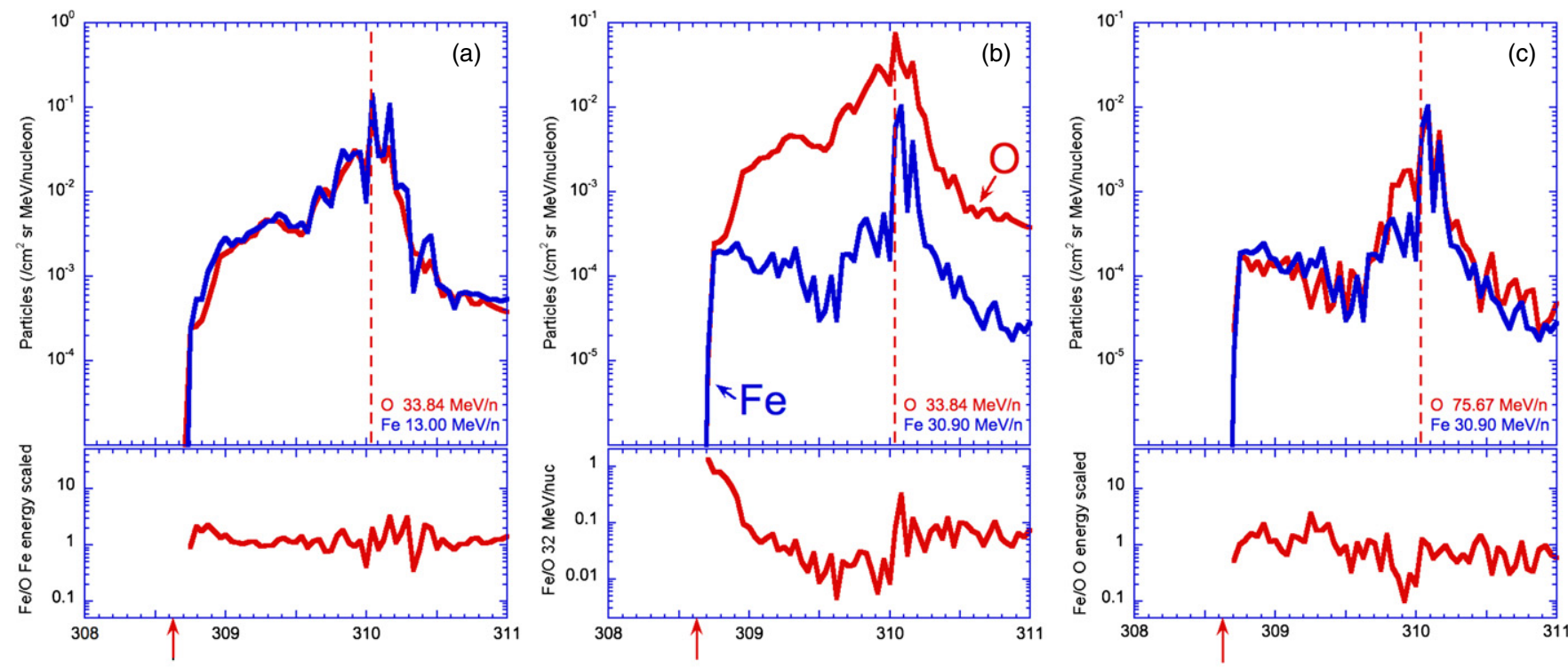

Figure 21. Hourly average $\mathrm{Fe}$ (blue) and $\mathrm{O}$ (red) for the 2001 November 4 (DOY 308) event: (a) $\mathrm{O}$ at reference energy, Fe at scaled energy; (b) both at $\sim 32 \mathrm{MeV}$ nucleon ${ }^{-1}$ reference energy; (c) Fe at reference energy, $\mathrm{O}$ at scaled energy (O intensity renormalized). Red arrow marks time of N06W18 X1/3B flare at 16:20 on November 4 (DOY 308); the dashed red line marks the time of the shock passage at $1 \mathrm{AU}$. Bottom panels: $\mathrm{Fe} / \mathrm{O}$ ratios for intensities in upper panels.

e.g., in Figure 11(b) for $\mathrm{O}$ and $\mathrm{Fe}$ in the same thin band at $1 \mathrm{AU}$, the broad distributions at injection for the two species are offset from each other. Figure 22(a) shows the size of this effect for the sample calculation described in Table 3. At low energies the injected energy of the Fe ions is $\sim 13 \%$ lower than the $\mathrm{O}$ ions, near the break the dependence is more complex, and toward higher energies the $\mathrm{Fe}$ and $\mathrm{O}$ have nearly identical initial energies as expected since the adiabatic deceleration is less important. Adiabatic cooling thus causes an energy shift in the two species, but it is small and not the same as the energy scaling parameter which removes the initial decrease in hourly $\mathrm{Fe} / \mathrm{O}$ ratios. Figure 22(b) plots the same ratio as in panel (a) except versus the $\mathrm{O}$ energy at injection, showing the very large effects at low energies where particles down to $50 \mathrm{keV}^{\text {nucleon }}{ }^{-1}$ at 1 AU originated above $\sim 200 \mathrm{keV}^{\text {nucleon }}{ }^{-1}$ at injection.

Cohen et al. (2007) have discussed in detail the use of energy scaling on the interpretation of SEP spectra in 14 events. In that analysis, the energy scaling referred to the spectral break energy (in contrast to the usage here where it was derived from the temporal changes of the $\mathrm{Fe} / \mathrm{O}$ ratio). For each SEP event an energy scaling factor was derived by finding the value which when applied to an element such as Fe minimized the energy dependence of the abundance with respect to $O$. The search for the optimum scaling factor for each event was subject to the condition that the low-energy abundance ratio (e.g., $\mathrm{Fe} / \mathrm{O}$ ) did not vary, and so by construction the derived abundances were closer to the low energy survey of Desai et al. (2006) than to previous surveys at higher energies. Remaining variations between different SEP events were attributed by Cohen et al. to other factors such as a variation in first ionization potential fractionation from one event to another. Cohen et al. (2007) also pointed out that previous surveys covering the range near $5 \mathrm{MeV}$ nucleon $^{-1}$ (Breneman \& Stone 1985; Reames 1995) were likely affected by the spectral rollover in their energy 

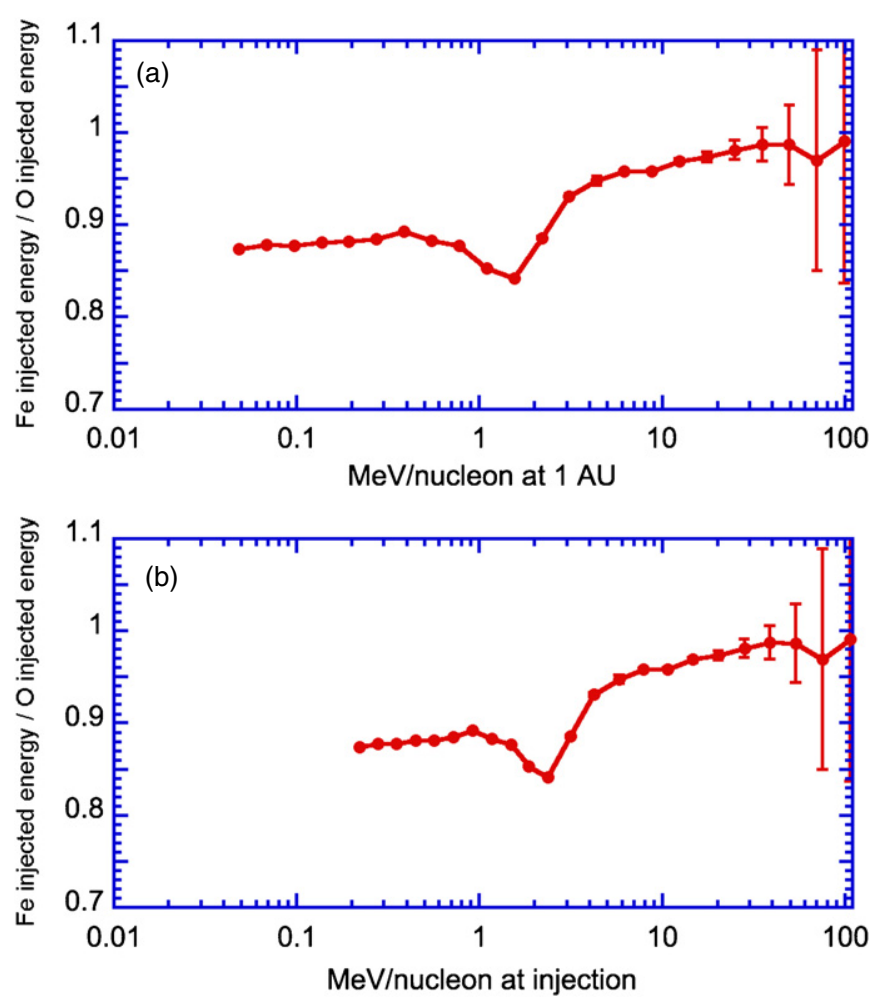

Figure 22. (a) Ratio of injected energy for Fe to injected energy for $\mathrm{O}$ for identical energy windows at 1 AU. Panel (b) same as (a) except points are plotted vs. the $\mathrm{O}$ energy at injection. Note that although the $1 \mathrm{AU}$ observations go down to $50 \mathrm{keV}$ nucleon ${ }^{-1}$, the mean particle energies at injection are all above $\sim 200 \mathrm{keV}^{\text {nucleon }}{ }^{-1}$.

range, which would have added a complicating factor to the averages reported.

\section{SUMMARY AND CONCLUSIONS}

The present survey of 17 western hemisphere large SEP events has examined intensity profiles and relative abundances of $\mathrm{H}, \mathrm{He}, \mathrm{O}$, and $\mathrm{Fe}$ over a broad energy range in order to explore whether the commonly observed decrease of $\mathrm{Fe} / \mathrm{O}$ during SEP event rise phases can be modeled as an interplanetary transport effect. While our earlier study (Mason et al. 2006) showed that energy scaling could decrease this temporal variation, the underlying physical reason was based only on simple arguments involving a particle's interplanetary diffusion coefficient. Here we have shown that a simulation model with release near the Sun followed by interplanetary transport including effects of focusing, convection, adiabatic deceleration, and pitch angle scattering also shows energy scaling as expected from the simple diffusion coefficient considerations, provided that the interplanetary turbulence is sufficiently high that significant pitch angle scattering occurs. Interplanetary propagation parameters derived for each event resulted in mean free paths similar to previous studies. The calculated intensity profiles fit the data reasonably; however, with deviations due to shock trapping, connection and corotation effects that were not in the model. The Fe/O ratio variation magnitude was reasonably consistent with the data. The model predicts that the $\mathrm{O} / \mathrm{He}$ ratio would also show a decrease during event rise phases, and this was confirmed, contradicting the expectation of no variation in $\mathrm{O} / \mathrm{He}$ if the early compositional properties were due to mixing of impulsive and shock-associated material. The model also predicts that the $\mathrm{He} / \mathrm{H}$ ratio would decrease during event rises, and this was observed only in some events and contradicted in others, identifying the temporal behavior of $\mathrm{H}$ as different from heavy ion species as has been argued previously ( $\mathrm{Ng}$ et al. 1999; Tylka et al. 1999; Reames et al. 2000).

Referring to the questions raised in the Introduction, we conclude:

1. The enhanced $\mathrm{Fe} / \mathrm{O}$ ratio observed during the rise phase in SEP events is generally a transport effect, which can be understood in terms of particle propagation in an IMF whose turbulence level is a power law in wavenumber. Hourly intensity profiles of SEPs in events with significant interplanetary scattering should be compared not at the same velocity $\left(\mathrm{MeV}\right.$ nucleon $\left.^{-1}\right)$ but rather at a scaled energy, which can be determined empirically by finding the scaling factor that removes most or all of the temporal variation in the rise phase hourly abundance ratios. The scaling factor for different elements compared to a reference element is given by Equation (1).

2. Energy spectra of SEPs are best organized also by using energy scaling determined empirically from the location of spectral break energies at $1 \mathrm{AU}$, and sized for different elements as given by Equation (1), as shown by other studies cited above. This removes much of the energy dependence of SEP abundances for different energies in a given event. The transport calculations here show that the spectral break must be a property of the injected spectra, and so must be due to acceleration and escape processes at injection. As such it is not the same scaling factor as that derived from the transport, which is due to properties of the interplanetary turbulence.

3. The scaling factors for both injection and transport follow Equation (1), but they reflect separate conditions at the acceleration site versus in interplanetary space. Scattering near a shock generally has much smaller diffusion coefficients than interplanetary space (e.g., $\mathrm{Ng}$ et al. 1999); however, Equation (1) depends not on the magnitude of the turbulence but rather the slope of the turbulence powerlaw spectrum. Since Equation (1) gives similar results for a wide range of turbulence power law indices (Figure 1), the scaling factors appropriate arising from transport and injection could often be similar, but they need not be.

4. SEP abundance ratios measured below the spectral break exhibit much less variation than those above the break due to differences in break energies for different elements, and differences of slope between different elements above the break. Therefore, abundances measured below the break are subject to fewer systematic uncertainties. Nevertheless, even below the break the relative abundances may bear the imprint of fractionation in the acceleration process, which would need to be understood to relate the $1 \mathrm{AU}$ energetic particle results to the source abundances.

5. Adiabatic deceleration of the SEPs is significant especially below $\sim 1 \mathrm{MeV}$ nucleon $^{-1}$ and is severe below $\sim 200 \mathrm{keV}^{\text {nucleon }}{ }^{-1}$ for events with moderate scattering. The deceleration does not change the abundance ratios by much, but it does lower the spectral break energy by typically a factor of $\sim 2$, which could be important for considering the acceleration and escape process. Below $\sim 1 \mathrm{MeV}$ nucleon $^{-1}$ the deceleration smears out the initial particle energies and so would tend to erase possible energy dependences in the source population such as in the ionization states. Additionally, particles injected below 
$\sim 200 \mathrm{keV}$ nucleon $^{-1}$ are cooled to the extent of being basically unobserved at $1 \mathrm{AU}$; therefore, when estimating the energy content of SEPs in an event to compare with the energy of an accelerating CME, it should be kept in mind that an injected spectrum below $\sim 200 \mathrm{keV}^{\text {nucleon }}{ }^{-1}$ is not known from $1 \mathrm{AU}$ observations.

Finally, we note from some of the cases examined in this paper that hourly abundance variations associated with interplanetary shock passages also can be decreased by applying energy scaling (Figures 2(f) and 21). This would be expected if the shock acceleration is due to particle scattering, as is generally believed to be the case. Since the scaling factor appropriate for the shock involves the acceleration physics it would be different from that coming from transport or SEP acceleration, nevertheless because of the weak dependence of the scaling on spectral index (Figure 1) it would not be surprising for this factor to be similar to that derived from the transport. Thus, in Figures 2(f) and 21, the energy scaling that decreases the SEP hourly abundance variations also decreases the variations around shock passage although clearly different physical mechanisms are at work. A more complete examination of interplanetary shock passages would be needed to explore this possibility further.

The particle simulations in this paper were carried out at the parallel processing facilities of the Center for Space Plasma and Aeronomic Research (CSPAR) at the University of Alabama in Huntsville. This work was supported at JHU/APL by NASA under grants NNX07AP69G (LWS TR\&T), and NNX10AT75G and NSF grant 1156138 . Work at Caltech is supported by grants NNX08AI11G and NNX11A075G; work at the University of Alabama in Huntsville is supported by NASA NNX07AP69G, NNX07AL52A, NNX09AP74A, and NSF ATM-0847719, and AGS-1135432, and work at SwRI is supported by NSF grants ATM-0550960 and ATM-0551127 and NASA grants NNG05GM88G, NNG05GQ94G, NNX07AC12G, NNX07AG85G， NNX07AP69G， NNX07AC15G， and NNX08AK87G.

\section{REFERENCES}

Anderson, K. A., \& Dougherty, W. M. 1986, Solar Phys., 103, 165 Aran, A., Sanahuja, B., \& Lario, D. 2006, Adv. Space Res., 37, 1240 Aran, A., Sanahuja, B., \& Lario, D. 2008, Adv. Space Res., 42, 1492 Axford, W. I. 1965, Planet. Space Sci., 13, 1301

Beeck, J., Mason, G. M., Hamilton, D. C., et al. 1987, ApJ, 322, 1052

Bieber, J. W., Matthaeus, W. H., Smith, C. W., et al. 1994, ApJ, 420, 294

Breneman, H. H., \& Stone, E. C. 1985, ApJ, 299, L57

Bruno, R., \& Carbone, V. 2005, Living Rev. Solar Phys., 2, 4

Cane, H. V. 1988, J. Geophys. Res., 93, 1

Cane, H. V., Mewaldt, R. A., Cohen, C. M. S., \& von Rosenvinge, T. T. 2006 J. Geophys. Res., 111, A06S90

Cane, H. V., Reames, D. V., \& von Rosenvinge, T. T. 1988, J. Geophys. Res., 93, 9555

Cane, H. V., von Rosenvinge, T. T., Cohen, C. M. S., \& Mewaldt, R. A. 2003, Geophys. Res. Lett., 30, 8017

Chollet, E. E., \& Giacalone, J. 2008, ApJ, 688, 1368

Chollet, E. E., Giacalone, J., \& Mewaldt, R. A. 2010a, J. Geophys. Res., 115, A06101

Chollet, E. E., Mewaldt, R. A., Cummings, A. C., et al. 2010b, J. Geophys. Res., 115, A10216

Cliver, E. W., Kahler, S., \& Reames, D. V. 2004, ApJ, 605, 902

Cohen, C. M. S., Mewaldt, R. A., Leske, R. A., et al. 1999, Geophys. Res. Lett., 26, 2697

Cohen, C. M. S., Mewaldt, R. A., Leske, R. A., et al. 2007, Space Sci. Rev., 130,183

Cohen, C. M. S., Stone, E. C., Mewaldt, R. A., et al. 2005, J. Geophys. Res., 110, A09S16

Desai, M. I., Mason, G. M., Gold, R. E., et al. 2006, ApJ, 649, 470
Dietrich, W. F., \& Tylka, A. J. 2001, in Proc. 27th Int. Cosmic Ray Conf., ed. K.-H. Kampert et al. (Göttingen: Copernicus), 3173

Dröge, W. 1994, ApJS, 90, 567

Dröge, W., Kartavykh, Y. Y., Klecker, B., \& Kovaltsov, G. A. 2010, ApJ, 709,912

Earl, J. A. 1974, ApJ, 188, 379

Earl, J. A. 1976, ApJ, 205, 900

Feit, J. 1973, Solar Phys., 29, 211

George, J. S., Lave, K. A., Wiedenbeck, M. E., et al. 2009, ApJ, 698, 1666

Gold, R. E., Krimigis, S. M., Hawkins, I. S. E., et al. 1998, Space Sci. Rev., 86,541

Goldstein, M. L., Fisk, L. A., \& Ramaty, R. 1970, Phys. Rev. Lett., 25, 832

Gosling, J. T. 1993, J. Geophys. Res., 98, 18937

Hasselmann, K., \& Wibberenz, G. 1968, Z. Geophys., 34, 353

Hovestadt, D., Klecker, B., Hoefner, M., et al. 1982, ApJ, 258, L57

Hudson, H. S., Fletcher, L., Kahn, J. I., \& Kosugi, T. 2004, in Solar and Space Weather Radiophysics, ed. D. E. Gary \& C. U. Keller (Astrophys. Space Sci. Library, Vol. 314; Berlin: Springer), 153

Isenberg, P. 1997, J. Geophys. Res., 102, 4719

Jokipii, J. R. 1966, ApJ, 146, 480

Jokipii, J. R. 1971, Rev. Geophys. Space Phys., 9, 27

Kahler, S. 1994, ApJ, 428, 837

Kahler, S. W. 2005, ApJ, 628, 1014

Kahler, S. W., Reames, D. V., \& Sheeley, N. R., Jr. 2001, ApJ, 562, 558

Kallenrode, M.-B., Cliver, E. W., \& Wibberenz, G. 1992, ApJ, 391, 370

Kartavykh, Y. Y., Dröge, W., Klecker, B., et al. 2007, ApJ, 671, 947

Klecker, B., Mewaldt, R. A., Oetliker, M., Selesnick, R. S., \& Jokipii, J. R. 1998, Space Sci. Rev., 83, 294

Klecker, B., Möbius, E., \& Popecki, M. 2006, Space Sci. Rev., 124, 289

Kocharov, G. E., Vainio, R., Kovaltsov, G. A., \& Torsti, J. 1998, Solar Phys., 182,195

Kocharov, L., Laivola, J., Mason, G. M., Didkovsky, L., \& Judge, D. L. 2008, ApJS, 176, 497

Kocharov, L., \& Torsti, J. 2002, Solar Phys., 207, 149

Kota, J., Manchester, W. B., Jokipii, J. R., de Zeeuw, D. L., \& Gombosi, T. I. 2005, in AIP Conf. Proc. 781, The Physics of Collisionless Shocks, ed. G. Li, G. P. Zank, \& C. T. Russell (Melville, NY: AIP), 201

Lario, D., Sanahuja, B., \& Heras, A. M. 1998, ApJ, 509, 415

le Roux, J. A., \& Webb, G. M. 2007, ApJ, 667, 930

Lee, M. A. 2005, ApJS, 158, 38

Li, G. 2008, in AIP Conf. Proc. 1039, Particle Acceleration and Transport in the Heliosphere and Beyond, ed. G. Li, Q. Hu, O. Verkhoglyadova, G. P. Zank, R. P. Lin, \& J. Luhmann (Melville, NY: AIP), 233

Li, G., Webb, G. M., le Roux, J. A., et al. 2009a, in Proc. 31st Int. Cosmic Ray Conf., ed. J. Szabelski \& M. Giller (Łodz: Univ. Łodz), 1361

Li, G., \& Zank, G. P. 2005, Geophys. Res. Lett., 32, L02101

Li, G., Zank, G. P., \& Rice, W. K. M. 2003, J. Geophys. Res., 108, 1082

Li, G., Zank, G. P., \& Rice, W. K. M. 2005, J. Geophys. Res., 110, A06104

Li, G., Zank, G. P., Verkhoglyadova, O., et al. 2009b, ApJ, 702, 998

Lin, R. P. 1987, Rev. Geophys., 25, 676

Mason, G. M. 1987, Rev. Geophys., 25, 685

Mason, G. M., Desai, M. I., Cohen, C. M. S., et al. 2006, ApJ, 647, L65

Mason, G. M., Gloeckler, G., \& Hovestadt, D. 1983, ApJ, 267, 844

Mason, G. M., Gloeckler, G., \& Hovestadt, D. 1984, ApJ, 280, 902

Mason, G. M., Gold, R. E., Krimigis, S. M., et al. 1998, Space Sci. Rev., 86, 409

Mason, G. M., Mazur, J. E., \& Dwyer, J. R. 1999, ApJ, 525, L133

Mason, G. M., Mazur, J. E., Dwyer, J. R., et al. 2004, ApJ, 606, 555

Mason, G. M., Nitta, N., Cohen, C. M. S., \& Wiedenbeck, M. E. 2009, ApJ, 700, L56

Mason, G. M., Reames, D. V., Klecker, B., Hovestadt, D., \& von Rosenvinge, T. T. 1986, ApJ, 303, 849

Mazur, J. E., Mason, G. M., Dwyer, J. R., et al. 2000, ApJ, 532, L79

Mazur, J. E., Mason, G. M., Klecker, B., \& McGuire, R. E. 1992, ApJ, 401, 398

McCracken, K. G., \& Rao, U. R. 1970, Space Sci. Rev., 11, 155

McGuire, R. E. 1983, Rev. Geophys., 21, 305

McGuire, R. E., von Rosenvinge, T. T., \& McDonald, F. B. 1986, ApJ, 301, 938

Mewaldt, R. A., Cohen, C. M. S., Giacalone, J., et al. 2008, in AIP Conf. Proc. 1039, Particle Acceleration and Transport in the Heliosphere and Beyond: 7th Ann. Int. Astrophys. Conf., ed. G. Li et al. (Melville, NY: AIP), 111

Mewaldt, R. A., Cohen, C. M. S., Labrador, A. W., et al. 2005a, J. Geophys. Res., 110, A09S18

Mewaldt, R. A., Cohen, C. M. S., \& Mason, G. M. 2006, in Geophys. Monograph 165, ed. N. Gopalswamy, R. A. Mewaldt, \& R. B. Torbert (Washington, DC: AGU), 115 
Mewaldt, R. A., Cohen, C. M. S., Mason, G. M., et al. 2005b, in AIP Conf. Proc. 781, Solar Energetic Particle Spectral Breaks, The Physics of Collisionless Shocks: 4th Ann. IGPP Int. Astrophys. Conf., ed. G. Li, G. P. Zank, \& C. T. Russell (Melville, NY: AIP), 227

Mewaldt, R. A., Looper, M. D., Cohen, C. M. S., et al. 2005c, in Proc. 29th Int. Cosmic Ray Conf., ed. B. Sripathi et al. (Mumbai: Tata Inst. Fundamental Research), 111

Möbius, E., Morris, D., Popecki, M. A., et al. 2002, Geophys. Res. Lett., 29, 1

Ng, C. K., \& Reames, D. V. 1994, ApJ, 434, 1032

Ng, C. K., Reames, D. V., \& Tylka, A. J. 1999, Geophys. Res. Lett., 26, 2145

Ng, C. K., Reames, D. V., \& Tylka, A. J. 2003, ApJ, 591, 461

Onsager, T., Grubb, R. N., Kunches, J., et al. 1996, Proc. SPIE, 2812, 281

Palmer, I. D. 1982, Rev. Geophys. Space Phys., 20, 335

Parker, E. N. 1965, Planet. Space Sci., 13, 9

Qin, G., Zhang, G., Dwyer, J. R., Rassoul, H. K., \& Mason, G. M. 2005, ApJ, 627,562

Qin, G., Zhang, M., \& Dwyer, J. R. 2006, J. Geophys. Res., 111, A08101

Ramaty, R., Paizis, C., Colgate, S. A., et al. 1980, in Solar Flares: A Monograph from the Skylab Solar Workshop II, ed. P. A. Sturrock (Boulder, CO: Colorado Associated Univ. Press), 117

Reames, D. V. 1995, Adv. Space Res., 15, 41

Reames, D. V. 1999, Space Sci. Rev., 90, 413

Reames, D. V. 2009, ApJ, 706, 844

Reames, D. V., Ng, C. K., \& Tylka, A. J. 2000, ApJ, 531, L83

Reid, G. C. 1964, J. Geophys. Res., 69, 2659

Rice, W. K. M., Zank, G. P., \& Li, G. 2003, J. Geophys. Res., 108, SSH 5

Roelof, E. C. 2000, in Acceleration and Transport of Energetic Particles Observed in the Heliosphere: ACE 2000 Symposium, ed. R. A. Mewaldt, J. R. Jokipii, M. A. Lee, E. Möbius, \& T. H. Zurbuchen (Melville, NY: AIP), 242

Ruffolo, D. 1995, ApJ, 1995, 861

Sarra, S. A. 2003, J. Online Math. Appl., 3, 389

Scholer, M., Hovestadt, D., Klecker, B., Gloeckler, G., \& Fan, C. Y. 1978, J. Geophys. Res., 83, 3349

Schulze, B. M., Richter, A. K., \& Wibberenz, G. 1977, Solar Phys., 54, 207
Skilling, J. 1971, ApJ, 170, 265

Stone, E. C., Cohen, C. M. S., Cook, W. R., et al. 1998a, Space Sci. Rev., 86,357

Stone, E. C., Frandsen, A. M., Mewaldt, R. A., et al. 1998b, Space Sci. Rev., 86,1

Tylka, A. J., Boberg, P. R., Cohen, C. M. S., et al. 2002, ApJ, 581, L119

Tylka, A. J., Cohen, C. M. S., Dietrich, W. F., et al. 2005, ApJ, 625, 474

Tylka, A. J., Reames, D. V., \& Ng, C. K. 1999, Geophys. Res. Lett., 26,2141

Verkhoglyadova, O., Li, G., Zank, G. P., et al. 2010, J. Geophys. Res., 115, A12103

von Rosenvinge, T. T., \& Reames, D. V. 1979, in Proc. 16th Int. Cosmic Ray Conf., ed. S. Miyake (Tokyo: Inst. Cosmic Ray Research), 68

von Rosenvinge, T. T., Richardson, I. G., Reames, D. V., et al. 2009, Solar Phys., 256,443

von Steiger, R, Schwadron, N. A., Fisk, L. A., et al. 2000, J. Geophys. Res., 105,27217

Wanner, W., \& Wibberenz, G. 1991a, in Proc. 22nd Int. Cosmic Ray Conf. (Vol. 3; Dublin: Inst. Adv. Studies), 221

Wanner, W., \& Wibberenz, G. 1991b, in Proc. 22nd Int. Cosmic Ray Conf. (Vol. 3; Dublin: Inst. Adv. Studies), 217

Webb, G. M., \& Gleeson, L. J. 1979, Ap\&SS, 60, 335

Wiedenbeck, M. E., Mason, G. M., Christian, E. R., et al. 2003, in AIP Conf. Proc. 679, Solar Wind Ten: Proc. 10th Int. Solar Wind Conf., ed. M. Velli, R. Bruno, \& F. Malara (Melville, NY: AIP), 652

Wiedenbeck, M. E., Mason, G. M., Cohen, C. M. S., et al. 2005, in Proc. 29th Int. Cosmic Ray Conf., ed. B. Sripathi et al. (Mumbai: Tata Inst. Fundamental Research), 117

Witte, M., Wibberenz, G., Kunow, H., \& Müller-Mellin, R. 1979, in Proc. 16th Int. Cosmic Ray Conf., ed. S. Miyake (Tokyo: Inst. Cosmic Ray Research), 79

Zank, G. P., Rice, W. K. M., \& Wu, C. C. 2000, J. Geophys. Res., 105, 25079

Zhang, G., Qin, G., \& Rassoul, H. K. 2009, ApJ, 692, 109

Zhang, M. 1999, ApJ, 510, 715 